\title{
Applications of metaplectic cohomology and global-local contact holonomy
}

\section{Walter J. Schempp ${ }^{1}$}

Received: 11 June 2020 / Revised: 5 July 2020 / Accepted: 7 July 2020 / Published online: 21 July 2020 (c) The Author(s) 2020

\begin{abstract}
The purpose of the paper is to elucidate by global-dual contact holonomy why one might expect the Foucault spherical pendulum's spin echo-stabilized, symplectic swing-plane through the rotation axis of the spinning earth to follow a parallel vector field as time passes at a velocity depending on the latitude of the swivel's location. The spinor geometric foundations of the paradigmatic Foucault spherical pendulum experiment of global-local contact holonomy exploit Hopf-Rinow type minimizing geodesic trajectories which appear as inertial traces designed by swing-planes on the terrestrial circular floor panel which is situated beneath the covariant motion of the oscillating pendulum device and where the linear traces are designing diameters of the spin structure induced Poincaré conformal hyperbolic open disc model. The quantum field theoretical evidence of the existence and uniqueness of categorizing parallel vector fields around smooth loops follows in terms of the open-book foliation with Hilbert space structures placed on the covariant pages.
\end{abstract}

Keywords Dynamic quantization and kinematic relativization - Hopf principal circle bundle $\cdot$ Non-local quantum information processing $\cdot$ Supplementary open-book foliation $\cdot$ Hilbert bundle Spin structure $\cdot$ Metaplectic Lie group Mp $(2, \mathbb{R}) \cdot$ The metaplectic coadjoint orbit model $\cdot$ Half-spinor Maslov index

Mathematics Subject Classification 81S10 - 70G65 - 70G45 - 57R17 - 53C80 . $53 \mathrm{C} 38 \cdot 53 \mathrm{C} 27 \cdot 53 \mathrm{C} 22 \cdot 53 \mathrm{C} 12 \cdot 22 \mathrm{E} 25 \cdot 14 \mathrm{H} 81 \cdot 11 \mathrm{E} 08 \cdot 11 \mathrm{G} 07 \cdot 11 \mathrm{R} 11$

Dedicated to the fourth centenary of the Kepplerian third spinor law $\mathrm{H}^{3}$ of planetary dynamics.

\footnotetext{
$凶 \quad$ Walter J. Schempp

schempp@mathematik.uni-siegen.de

1 Lehrstuhl für Mathematik I, Universität Siegen, 57068 Siegen, Germany
} 


\section{Dynamic quantization and kinematic relativization}

It is a highly remarkable feature of the Copernican lecture on the physical world that the diurnal rotation of the spinning earth, in contrast to its annual motion around the central body, which it shares with the orbits of the other planets of the system and in which it merely takes over what had previously been the mobile sun's central position, is a novelty in the Copernican heliocentric reform idea of physical astronomy. The replacement does not appear in the classical cosmology by the Ptolemaic codification of geocentrism and would be inexplicable in the framework of its ordo orbium and the relativistic legitimacy of its stellar kinematics, which means the disclosure of the relations of order in the universe. The formulation according to which the earth possesses the phenomenon of diurnal rotation not only principally and by its nature but also "without the influence" of other factors is based on this unique singularity of mathematical astrophysics [56].

Seen under the perspective of the Kepplerian planetary contact holonomy, the problem of describing metaplectically the planetary orbits around the sun, the immobile center of the planetary system, would have offered the most precise points of reference in post-Copernican mathematical astrophysics because in the phenomenal motions of the planets two sources of covariant phase factors are engaged: Their real eigenmotions on the planetary orbits around the sun, and the projection of the earth's real eigen-motion, in its annual revolution and in diurnal rotation, onto the former stellar movements of heliocentrism. Nicolaus Copernicus (1473-1543), as a successor to the Pythagoreans, first hit upon the possibility of substituting the spinning earth's axial eigen-motion for the diurnal rotation of the heavens of the fixed stars in order to establish the relativistic legitimacy of the stellar time scale associated with the ordo orbium of the universe. In this way, Copernicus became the protagonist of the new idea of the ordo orbium not so much because he replaced one world-model with another, and thus demonstrated what radical incisions into the substance of the tradition were possible, as because he established an absolutely novel and universal claim to cosmic truth. Giordano Bruno (1548-1600), the "speculum of infinity", needed for his understanding the projective Copernicanism as truth. Thus he should be considered as one of the earliest spearheads of projective geometry.

It needed more than 300 years to visualize the Copernican construction of the diurnal rotation per sidereal day of the spinning earth. The impressive spherical pendulum holonomy experiment designed by Jean Bernard Léon Foucault (1819-1867) took place in 1851 beneath the dome of the Panthéon, Paris, where he suspended a 28$\mathrm{kg}$ brass-coated lead bob with a 67-m-long wire on the solid ball. For this choice of angular gauge function of relativistic gravitation, at the reference circle of $\varphi_{0}=+41^{\circ}$ $\mathrm{N}$ Latitude direction angle, the $\varphi_{0}$-latitudinal phase angle displacement $\Delta \theta$ of the spin echo-stabilized, symplectic swing-plane, which is caused by the parallel transport or parallel translation of the initial, symplectic swing-plane along the latitude of the terrestrial globe $\mathbb{S}_{2} \cong \operatorname{SO}(3, \mathbb{R}) / \mathrm{SO}(2, \mathbb{R}) \cong \mathbb{P}_{1}(\mathbb{C})$, is about $11^{\circ}$ per hour, or about $272^{\circ}$ per sidereal day. The formulation of quantum mechanics requires the use of complex numbers to make sense of phase factors of coherent wavelets. For the latitude direction angle $\varphi \in] 0, \pi$, a rigorous mathematical treatment of the paradigmatic Foucault spherical pendulum experiment of dual contact holonomy in terms 
of the geodesic curvature form $k_{\gamma}$ of the $\varphi$-latitudinal smooth loop $\gamma$ in the supporting absolute Riemann contact sphere $\mathbb{S}_{2}^{\frac{1}{2}} \hookrightarrow \mathbb{P}_{\mathbb{C}}\left(\mathfrak{L} \mathfrak{i} \mathfrak{e}(\mathcal{N})^{*}\right)$ of radius $\frac{1}{2}$ can be based on the de Coriolis alternating trilinear form of inertial force field. It relies on the equivalence principle of gravitational and inertial mass, and the temporal-spatial relativistic signature $(1,3)$ of the Lorentz-Möbius Lie group $\operatorname{PSO}(1,3, \mathbb{R})$ of even Witt index 4 and rank 8 . In fact, every four-dimensional Lorentz frame is the image of the four-dimensional canonical Lorentz frame by a unique element of $\operatorname{PSO}(1,3, \mathbb{R})$. This describes a transitive and proper left action of $\operatorname{PSO}(1,3, \mathbb{R})$, and an analogous right action as well.

One notes that the Lorentz-Möbius Lie group $\operatorname{PSO}(1,3, \mathbb{R})$ forms the connected component of the unit element in the Lorentz group $\mathrm{O}(1,3, \mathbb{R})$. The special Lorentz group $\mathrm{SO}(1,3, \mathbb{R})$ is the subgroup of index two in $\mathrm{O}(1,3, \mathbb{R})$ of endomorphisms of the vector space $\mathbb{R}^{(1,3)}$ of Minkowski time-space signature $(1,3)$. Using orthonormal frames it can be seen that all Minkowski time-spaces are ismorphic so one can speak of the Minkowski time-space. Since $\operatorname{SO}(1,3, \mathbb{R})$ also preserves the orientation, the Lorentz-Möbius Lie group $\operatorname{PSO}(1,3, \mathbb{R})$ is the subgroup of index two in $\mathrm{SO}(1,3, \mathbb{R})$ of endomorphisms of the Minkowski time-space $\mathbb{R}^{(1,3)}$ which preserve the axial future/past double light-ray cone of lightlike vectors, and the orientation.

Due to the actions on the principal $\operatorname{PSO}(1,3, \mathbb{R})$-Lorentz frame bundle of relativity over $\mathbb{P}_{\mathbb{C}}\left(\mathfrak{L} \mathfrak{i} \mathfrak{e}(\mathcal{N})^{*}\right)$ [66], the classical algebraic theory of spinors and associative Clifford/Graßmann algebras of orbital angular momenta or torques suggests to study the representational interplay with the closely tied non-associative alternative normed division algebra of octonions $\mathbb{O}$ [42], where

$$
\operatorname{dim}_{\mathbb{R}} \mathbb{O}=2^{3}=8
$$

In view of the Galois cohomological origin of the concept of quantum entanglement, the cyclic Galois extension of degree 2 of the groundfield $\mathbb{R}$ and associated with the division algebra of classical quaternions $\mathbb{H} \cong \mathbb{C} \times \mathbb{C}$ remained outside a mathematically rigorous treatment of quantum information processing. Similarly the relevance of the non-associative division algebra of octonions $\mathbb{O} \cong \mathbb{H} \times \mathbb{H}$ remained rather obscure until the discovery of the third order principle of spinor triality took place which exploits the symmetry between vectors and spinors in eight-dimensional Euclidean vector space [10].

A far-reaching generalization is the third order principle of triality of the algebraic theory of spinors and associative Clifford algebras on a par with Graßmann or exterior algebras of orbital angular momenta or torques. The interplay of their representations includes the Kepplerian spinor laws of planetary dynamics. The third order principle of spinor triality needs to go deeply into the structure of the octonion projective manifold $\mathbb{P}_{\mathbb{O}}\left(\mathfrak{L i e}(\mathcal{N})^{*}\right)$ and its octonionic foliation

$$
\mathbb{P}_{\mathbb{O}}\left(\mathrm{T}_{\bullet}\left(\mathbb{S}_{8}\right)\right) \hookrightarrow \mathbb{P}_{\mathbb{O}}\left(\mathfrak{L i e}(\mathcal{N})^{*}\right)
$$


on which it is defined. In this context, the sphere $\mathbb{S}_{8} \cong \mathbb{P}_{1}(\mathbb{O})$ forms the compact base manifold of the Hopf principal sphere bundle

$$
\mathbb{S}_{7} \hookrightarrow \mathbb{S}_{15} \stackrel{\eta_{8}}{\longrightarrow} \mathbb{S}_{8}
$$

with Hopf projector $\eta_{8}: \mathbb{S}_{15} \longrightarrow \mathbb{S}_{8}$. It is related to the second supplementary theorem of the famous quadratic reciprocity law [46].

There is hardly another event in the history of science about which such mutually exclusive theses have been set up as the transformation of astrophysics into a form that did not block the Copernican heliocentric reform idea and the quantum field theoretic development of an inclinatio contact geometry of inertia consisting of supporting two-sided tangent planes. The underlying principle of circular inertia derives from the geodesics with respect to the Poincaré metric of the open disc model. It is based on the mathematical concept of spinor which permits a transition from gravitation physics to the non-invasive imaging modality of nuclear spin magnetic resonance tomography with its own specific vocabulary $[9,44,45]$. The transition has been anticipated by Johannes Keppler (1571-1630) who referred his mathematical astronomy to William Gilbert's treatise De magnete in 1600, amost 90 years before the appearance of Isaac Newton's unifying principle of gravitation in his Philosophice Naturalis Principia Mathematica. A misguided and sometimes unwitting interpretation of Keppler's profound astrophysics as a precursor to Newton's has impeded any fair analysis of it [56].

An obstacle to the appreciation of the common underlying third order principle of spinor triality has been the broad spectrum of different applications, for detection of its presence in one place need not necessarily suggest its presence elsewhere. In fact, investigators in one seemingly isolated special field may very well never have been aware that the third order principle of spinor triality had been found in some different field seemingly not related to theirs. Another factor certainly contributory to its relative obscurity is that the third order principle of spinor triality is not in fact one object, but a collection of similar objects, rather like the Heisenberg unipotent Lie group $\mathcal{N}$, or the compact, triality conformally triangulated, homogeneous Heisenberg nilmanifold $\Lambda \backslash \mathcal{N}$, or overlapping functors. or even a combination of several schemes in the sense of algebraic geometry. Thus one has to look with a certain pair of spectacles in order to see the very diversity of the topics on the list of different applications as being united via a single phenomenological structure.

\section{Computational neuroscience: Representation of visuospatial states}

Exploring the quantum superposition states of macroscopic objects provides an intriguing avenue to study a suite of quantum-enhanced technologies. One prominent route of investigation is provided by the non-invasive imaging modalities of diffusion nuclear spin magnetic resonance tomography and human connectome tractography which have tremendous radiological potential for the understanding of biological 
tissue properties in vivo across a wide range of organs. The introduction of the technique of diffusion-weighting of pulsed magnetic field gradient trains into the armoury of quantitative nuclear spin magnetic resonance tomography specifically allows elucidating brain structure and cerebral microcircuitry in health and disease [30,48,57,64]. In fact, the natural diffusion of tissue protons ${ }^{1} \mathrm{H}$ can be used in the fields of nuclear spin magnetic resonance tomography and fiber tracking as the supplementary contrast mechanism of diffusive spin transport. The inclinatio contact geometry of tissue protons ${ }^{1} \mathrm{H}$ underlying the classical Stejskal-Tanner diffusion spectroscopy filter bank construction permits to perform series of non-invasive, precise, reproducible measurements of diffusive spin tansport by virtue of the spin echo arising in the presence of time dependent balanced linear magnetic field gradients.

In the field of diagnostic radiology, the key concept of spin is conceived as internal angular momentum [22,29,57]. Today, diffusion-weighting in nuclear spin magnetic resonance tomographic pulsed gradient trains is an almost exclusively used filter bank technique of non-invasive neuroanatomic tissue imaging for application in neurological diagnosis and psychiatric and neurological research, in order to locate brain tumors, depict an assessment of their invasivity evolution, and assist in the neurosurgical planning of focal epilepsy surgery $[7,64]$. Kepplerian precision affiliated with the spin echo-validation of the basic Heisenberg uncertainty principle infra imply that the ratio orbium of the tractographic reconstruction by spiral readout is paramount in neurosurgery because surgical injury to the cerebral white matter tracts can lead to permanent neurological deficits. In addition to this basic source of uncertainty, a source of error for tractography is in the inference of fiber orientation $[62,63]$. The inference is fundamentally challenging, as the resolution of diffusion nuclear spin magnetic resonance tomographic data is much more course than the neuroanatomic architecture of interest [21].

The navigational problem of keeping track of the environmental aspects requires some insight into the neuronal architecture and the structure of the microcircuitry of the cerebral formations $[3,15,19,75]$. Within the limbic lobe, neuroanatomically located on both sides of the thalamus, immediately beneath the lobus temporalis medialis of the cerebrum primarily in the midbrain, the medial entorhinal cortex cerebri forms a key relay structure for neural connections between the perirhinal and postrhinal cortices and prosubiculum, presubiculum, subiculum and parasubiculum as parts of the dorsal hippocampus which mediates much of the visuospatial input information [58,59]. In fact, the area entorhinalis of deep learning, despite its small size, is the principal input of high resolution information to the hippocampus $[13,33]$. Concerning the geometric strategies of visuospatial state representation and navigation, the third order principle of spinor triality forms the hub in the field of computational neuroscience by providing evidence for the neuroanatomic ratio orbium $\frac{3}{2}$ of space sensitively firing lattice neurons in the dorsolateral band of the entorhinal-hippocampal cortex cerebri which performs, as a part of the lobus temporalis medialis of the limbic lobe, visuospatially memorized navigation control $[12,13,57]$. The functional differences between dorsal and ventral hippocampus reflect the recurrent connectivity with modules of the entorhinal cortex cerebri that are differently linked to the rest of the cortex cerebri in 
the cerebral realization of a global position circuitry encoded by spacially sensitive entorhinal lattice neurons or $\Gamma$-neurons $[14,49]$.

Theorem 1 The entorhinal lattice neurons or $\Gamma$-neurons firing on the compact, triality conformally triangulated, homogeneous Heisenberg nilmanifold $\Lambda \backslash \mathcal{N}$ of right cosets modulo the cocompact, discrete subgroup $\Lambda \hookrightarrow \mathcal{N}$ encode equivariantly the triality conformal, neuronal, modular organization of the environmental octonion projective manifold $\mathbb{P}_{\mathbb{O}}\left(\mathfrak{L i e}_{\mathfrak{e}}(\mathcal{N})^{*}\right)$ of visuospatial states. It envelops the area entorhinalis of the cortex cerebri in the lobus temporalis medialis of the cerebrum and affords the realignment of the visuospatial memory readout process within the hippocampus, which is embedded into the lobus temporalis with its neuronal projections onto the hippocampal area within the limbic lobe.

The neural modular organization of the entorhinal-hippocampal cortex cerebri architecture under its recurrently connected bands acts parallel to the sulcus rhinalis and cuts across the medial and lateral subdivions of the area [12,75]. In terms of the laminar connectome, it stands out as the primary interface between the extensively connected hippocampus and the neocortex and includes the doubly covered, non-cyclic hexagonal lattice realization $\Lambda \hookrightarrow \mathcal{N}$ representing the triangular neuroanatomic ratio orbium. In view of the configurational $\frac{\text { hexagon }}{\text { square }}$ lattice design of computational neuroscience, realignment of the Poisson sum projection onto the compact, triality conformally triangulated, homogeneous Heisenberg nilmanifold $\Lambda \backslash \mathcal{N}$, a principal circle bundle

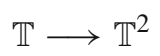

over the two-dimensional compact torus group $\mathbb{T}^{2} \cong \mathbb{R} \oplus \mathbb{R} / \mathbb{Z} \oplus \mathbb{Z}$ with discrete spectrum, implements via duality in terms of the triality conformal triangulation $\Lambda$ a hyperbolic location code over long terms and large spatial ranges [14,16]. Thus it is the profoundness of the spinor triality theorem which establishes a broader platform for the field of experimentally supported computational neuroscience by providing a universal coordinatization for navigational purposes of the fibering $\mathbb{Z}^{3} \backslash \mathcal{N} \longrightarrow \mathbb{T}^{2}$ with fiber the circle group $\mathbb{S}_{1}$ of the compact homogeneous nilmanifold $\Lambda \backslash \mathcal{N}[35,36,53,75]$.

Ultra high-field nuclear spin magnetic resonance tomographic views up to 9.4 Tesla permit to justify the doubling strategy by dividing the intrahippocampal circuitry into two pathways, the polysynaptic pathway, which links all parts of the hippocampus by a long neuronal chain, and the direct pathway, which directly reaches the output neurons of the hippocampus $[4,12]$. Lesions of projective connections of the area entorhinalis of the cortex cerebri to the hippocampus and excitotoxic damage to the parahippocampal cortex cerebri, which is engaged in the encoding and recognition of environmental scene representations in the octonion projective manifold $\mathbb{P}_{\mathbb{O}}\left(\mathfrak{L} \mathfrak{i}(\mathcal{N})^{*}\right)$, give rise to pronounced impairment of visuospatial learning and consolidation of semantic memory, episodic memory, and spatial memory of morbus Alzheimer pathology [23,52,65]. The semantic memory involves memory of facts and concepts; the episodic memory permits conscious recollection of events and the relations between them; the spatial memory involves spatial location recognition. Thus, it should be noted that the hippocampal allocortex, composed of only three layers of the limbic lobe, has very high 
cognitive function. Finally, the hippocampus can generate new neurons throughout life.

The data of nuclear spin magnetic resonance tomographic imaging can be coregistered and overlaid on the live view of the patient's brain during surgery using image-guided navigation software. Preoperative tractography in conjunction with the interoperative live view of the patient, provide important information to the neurosurgeon to plan the optimal neurosurgical approach to minimize injury to the surrounding healthy cerebral structures [7].

In the case of ischemic human stroke at the acute cerebral infarction stage, a region of the human brain with higher signal intensity than healthy tissue demonstrates decreased diffusion, the region of the infarct. In the clinical study of diffusion anisotropy, the nerve fiber tract directions in cerebral white matter have directional diffusion and different enhancement depending on the direction of the diffusionsensitizing gradient $[21,30]$. These directional phenomena are used in the technique of fiber tracking. Diffusion connectome nuclear spin magnetic resonance tractography, a computational reconstruction method based on diffusion-weighted nuclear spin magnetic resonance tomography, attempts to reveal the connection trajectories of cerebral white matter pathways in vivo and to infer the underlying structural connectome of the white matter architecture of the human brain $[8,28]$.

The predominant effect of nuclear spin magnetic resonance spectroscopy is the rephasing phenomenon of spin echo. In the mid-1960s, the basic idea was to insert pulsed gradients of inclinatio contact geometry into the protocol of basic spin echo trains, which resulted in much improved sensitivity to diffusion in comparison to the steady state gradients used previously in nuclear spin magnetic resonance tomography. Modern diffusion-weighted trains all trace their origin to the pulsed gradient spin echo technique developed by Stejskal and Tanner $[54,55,60]$ for the spin diffusion of biological tissue protons ${ }^{1} \mathrm{H}$. Strong bipolar diffusion-sensitizing magnetic field gradients are applied on either side of the $180^{\circ}$-pulse so that diffusion nuclear spin magnetic resonance tomography has been the key driver in the development of strong gradient technologies, providing higher and higher gradient amplitudes [11,32]. The phases of stationary spins are unaffected by the diffusion-sensitizing gradient pair of dephasing and rephasing graduents, since any phase accumulation from the first gradient lobe is reversed by the second one. Diffusing spins, however, move into different locations between the first and second lobes, falling out of phase and losing signal intensity as in binary pulsar astrophysics infra.

Immediately following the last diffusion-sensitizing gradient, an image acquisition modality is played out. This is typically an echo-planar imaging train using rapidly oscillating phase and frequency gradients that generate multiple gradient echoes $[24,32,47]$. In clinical nuclear spin magnetic resonance tomography, ultrafast image acquisition modality is generally required to minimize the effects of bulk motion such as vascular pulsations on the diffusion-weighted page readout [67]. Other modalities such as fast spin echo are possible, but are not as widely used for clinical protocols of adequate imaging quality at the present time. Both ultrastrong magnetic field gradients and echo-planar readout strategies have improved the robustness and utility of diffusion nuclear spin magnetic resonance tomography since its inception. 
The volume of typically voxels is in the cubic millimeter range, allowing the passage of many thousands of axonal bodies through each of the measured regions. Studies of the relevant half-spinor Maslov index are therefore limited to the investigation of gross-scale fiber bundles that are composed of a large population of axons, each of which contributes to the diffusion nuclear spin magnetic resonance tomographic signal [25]. This chasm between the dimensions of the source of the signal cell bodies and the spatial resolution leads to uncertainty in the interpretation of the voxel-by-voxel signal, which is amplified in the diffusion-weghted tractography experiment.

Connectome filter bank research Nerve fiber bundles are non-invasively displayed by diffusion-weighted nuclear spin magnetic resonance tractography of the human brain within the octonion projective manifold $\mathbb{P}_{\mathbb{O}}\left(\mathfrak{L i \mathfrak { i }}(\mathcal{N})^{*}\right)$ of visuospatial states. The modality of diffusion-weighted tractography exploits the third order symmetries of the projective manifold $\mathbb{P}_{\mathbb{O}}\left(\mathfrak{L i e}(\mathcal{N})^{*}\right)$. In terms of mathematical phenomenology, the manifold $\mathbb{P}_{\mathbb{O}}\left(\mathfrak{L} \mathfrak{i}(\mathcal{N})^{*}\right)$ represents the foundational link between the physical world and the projective visuospatial life-world.

The invariantly supported cubic form and non-degenerate quadratic form of the third order principle of spinor triality define the ratio orbium in terms of the uniform pulse sequence duration and the amplitude of the dephasing gradient, respectively. The ratio orbium increases linearly with the number of inidual bipolar pulses applied prior to the $180^{\circ}$-pulse. Since the off-diagonal elements of the diffusion tensor cancel when all gradient effects are superimposed, multiplication of the inverse ratio orbium with the squared gyromagnetic ratio and the averaged diffusion coefficient of human brain neuroanatomic tissue in vivo produces the signal response attenuation filter bank of the pulsed gradient spin echo experiment of diffusion-weighted nuclear spin magnetic resonance tomography in terms of the interval length between the onsets of the individual gradients which are acting as a control parameter. Taking into account of the realignment isomorphy of time course by the central projective collineations or perspectivities

$$
\operatorname{PSO}(1,1, \mathbb{R}) \cong \mathbb{R}
$$

of intersecting or different parallel planes of the Minkowski time-space $\mathbb{R}^{(1,3)}$, the spherical deconvolution kernel admits ratio orbium symmetry of the third order principle of spinor triality of the algebraic theory of spinors and associative Clifford algebras on a par with Graßmann or exterior algebras of orbital angular momenta within the octonion projective manifold $\mathbb{P}_{\mathbb{O}}\left(\mathfrak{L} \mathfrak{i} \mathfrak{e}(\mathcal{N})^{*}\right)[62,63]$. In accordance with the phenomenological description of visuospatial dynamics, the octonion projective manifold $\mathbb{P}_{\mathbb{O}}\left(\mathfrak{L} \mathfrak{i} \mathfrak{e}(\mathcal{N})^{*}\right)$ represents the foundational link between the physical world and the projective visuospatial life-world.

\section{From neuroanatomic network organization to spin spectroscopic astrophysics}

Looking back at the epoch-making chief work De revolutionibus orbium colestium, Libri VI of 1543, the "neo-Pythagorean" Copernicus had been satisfied to deduce the 
mere possibility of the spinning earth's motion from its spherical form. At the latest, the Copernican reform idea of the spinning earth would have made its breakthrough with Foucault's famous spherical pendulum holonomy experiment of 1851 and the mathematical study of the parallel transport dynamics of its symplectic swing-planes, bound to a rigid sphere, to achieve the projective stellarization of the spinning earth. Galileo Galilei (1564-1642) himself felt to be the greatest triumph of his phenomenology: that he made the spinning earth a star among the other stars inside the ordo orbium; he had gone far beyond Copernicus in that he had given the earth itself the astrophysical quality of a moving planet. The computational expertise that Keppler has visually collected in the Mysterium cosmographicum of 1597 for an understanding the ordo orbium did not weight for him his profound mathematical insights into the stellarization of the spinning earth as a remarkable astrophysical piece of finding (Fig. 1).

As described exhaustively in the epoch-making treatise Astronomia nova of 1609, Keppler's intention was to advocate and defend the Copernican reform and then to proceed further in the direction that Copernicus had entered upon with his revival of the bold idea of global cosmic symmetry. It goes beyond obedience to the Platonic requirement insofar as symmetry motivates to overcome the isolated representation of individual planetary orbits in order to design the common relation between them in accordance with the third Kepplerian spinor law $\mathrm{H}^{3}$ of Harmonices mundi of 1619 that embraces the swiveling processes of all the planets of the ordo orbium into their spherically calibrated contact configurations. The forestalled idea of a cosmic supersymmetry obviously contradicts the Platonic postulate of uniform revolutions. Consequently Keppler's Harmonices mundi provides the viewpoint appropriate for backstage inspection of the dynamics of the Foucault spherical pendulum experiment and its dual contact holonomy. For Copernicus himself, the Platonization formula that describes his work as "having solved the problems of the heavens by not looking at the heavens" appears as an exhibition of the cosmic truth (Fig. 2).

In the prehistory of the spherical pendulum holonomy experiment, it was the pervasive idea of the polymath Gottfried Wilhelm Leibniz (1646-1716) of including the less accessible temporal-süatial aspects into the ordo orbium of the universe $\mathbb{P}_{\mathbb{C}}\left(\mathfrak{L i e}(\mathcal{N})^{*}\right)$ and extirpating the heliocentric truth question in order to complete the projective optics of the temporal-spatial totality for every individual reference position by the quadratic extension concept of kinematic relativization. As a realization of the hyperquadric in the vector space $\mathbb{C}^{2} \cong \mathbb{R}^{4} \hookleftarrow \mathbb{S}_{3}$, the $(2+1)$-dimensional real Heisenberg Lie algebra $\mathfrak{L i} \mathfrak{e}(\mathcal{N})$ of nilpotent matrices

$$
\left\{\left(\begin{array}{ccc}
0 & w & \frac{z}{2 i} \\
0 & 0 & \bar{w} \\
0 & 0 & 0
\end{array}\right) \mid w \in \mathbb{C}, z \in \mathbb{R}\right\}
$$

supports the post-Copernican conclusion of the relativistic equivalence of the worldsystems, which has been described earlier in comparison to an absolute knowledge of system relativization by the reformator Andreas Osiander (1498-1552) in his mostcriticized unauthorized preface Ad lectorem of the Copernican revolutionary treatise De revolutionibus orbium calestium on the reform idea of heliocentric cosmology. 


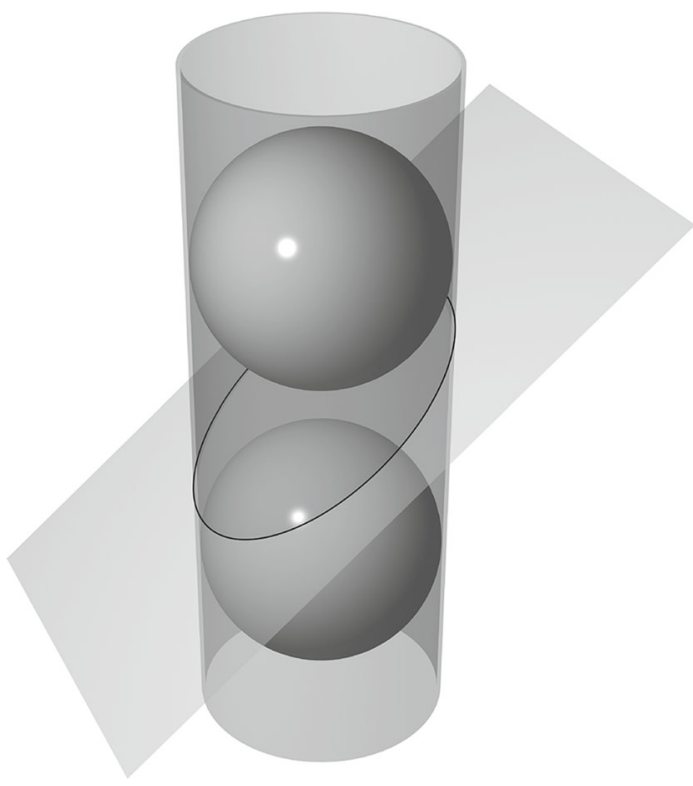

Fig. 1 Inclinatio contact geometry within the Picard torus: Swiveling action of pure spinors on the twofold spherically calibrated contact configuration affords covariant metaplectical visualization of a global planetary orbit via Kepplerian planetary contact holonomy. The inclined two-sided contact plane supporting as a real projective quadric of rank two the compact spin manifold $\mathbb{S}_{2}^{\frac{1}{2}} \times \mathbb{S}_{2}^{\frac{1}{2}}$. The norm spheres give rise to the real foci of the Kepplerian orbital ellipse within the circular cylinder $\mathfrak{Z}^{\mathfrak{c}} \hookrightarrow \mathfrak{L} \mathfrak{i}(\mathcal{N})^{*}$ and axis $\mathfrak{L i e}(\mathcal{Z})^{*}$. Actually an ellipse which does not design a circle has four different focal points in the complex projective manifold $\mathbb{P}_{\mathbb{C}}\left(\mathfrak{L i e}(\mathcal{N})^{*}\right)$, two of which are not real and located on the minor axis and symmetrically situated with respect to the center of the orbital ellipse. Inertia implies the existence of a second inclined twosided tangent plane supporting as a real projective quadric of rank two the split spin manifold $\mathbb{S}_{2}^{\frac{1}{2}} \times \mathbb{S}_{2}^{\frac{1}{2}}$ in symmetric contact position and passing through the center. Witt index two calibration of the equiareal action of the metaplectic Lie group $\mathrm{Mp}(2, \mathbb{R})$ on the two-fold contact configuration in the complex universe $\mathbb{P}_{\mathbb{C}}\left(\mathfrak{L} \mathfrak{i} \mathfrak{e}(\mathcal{N})^{*}\right)$ visualizes the second Kepplerian spinor law of planetary motion around the central body, whereas the spherical volume calibration of the action of the Witt spinor norm group WS(R) on the double contact configuration of odd pure half-spinor space $\mathfrak{S}_{-}$and even pure half-spinor space $\mathfrak{S}_{+}$in the octonion universe $\mathbb{P}_{\mathbb{O}}\left(\mathfrak{L i} \mathfrak{e}(\mathcal{N})^{*}\right) \hookleftarrow \mathbb{P}_{\mathbb{C}}\left(\mathfrak{L} \mathfrak{i}(\mathcal{N})^{*}\right)$ gives rise by virtue of the successive applications of radial codifferentials $\delta$ on Stokes' theorem for the oriented volume form of the three-dimensional solid ball, to the third Kepplerian spinor law $\mathrm{H}^{3}$ of the dynamics of the heliocentric system. The spherical contact $\frac{3}{2}$-power calibration geometry accounts for the two-fold covering group $\mathrm{SO}(4, \mathbb{R})$ of the "double" product Lie group of spatial rotations $\mathrm{SO}(3, \mathbb{R}) \times \mathrm{SO}(3, \mathbb{R}) \cong \operatorname{Aut}(\mathbb{H}) \times \operatorname{Aut}(\mathbb{H})$ to force the planets upon their swiveling processes into the three Kepplerian spinor laws of the ordo orbium governed by the action of the compact spinor group $\operatorname{Spin}(4, \mathbb{R}) \cong \mathbb{S}_{3} \times \mathbb{S}_{3} \cong \operatorname{Spin}(3, \mathbb{R}) \times \operatorname{Spin}(3, \mathbb{R}) \cong \operatorname{SU}(2, \mathbb{C}) \times \operatorname{SU}(2, \mathbb{C})$

The Aristotelian Philipp Melanchthon (1497-1560) shared in several editions of his influential treatise Initia doctrince physica in 1549 the critical viewpoint of Osiander and emphasized in the chapter with the concise title Quis est motus mundi? that Copernicanism represents more than a scientific shenanigans and actually forms a part of human consciousness. It is not phenomenological schools, terminologies, or philosophical systems that are in competition, but concepts of scientific truth. 


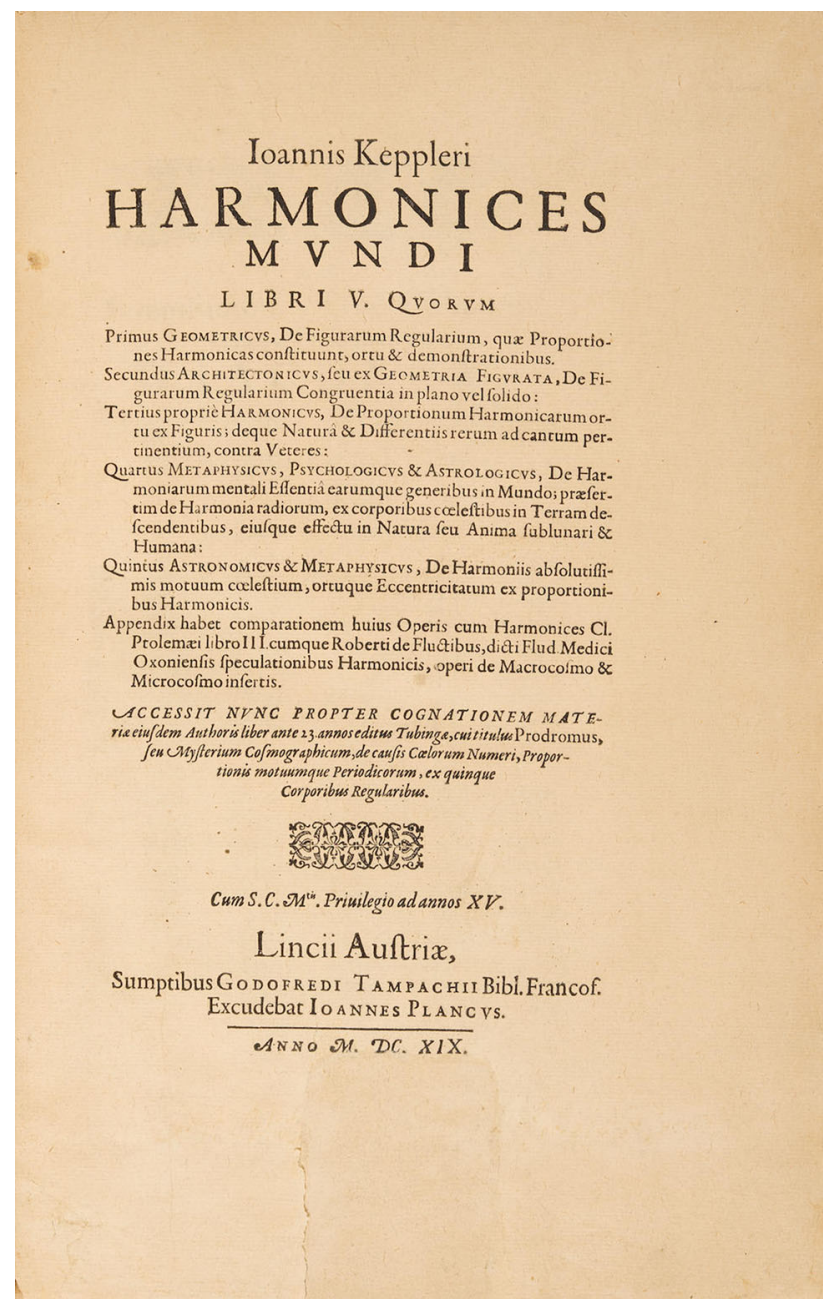

Fig. 2 Four hundred years of mathematical culture: The epoch-making treatise "Harmonices mundi. Libri V" of the mathematician and astronomer Johannes Keppler 1619. The Kepplerian third spinor law $\mathrm{H}^{3}$ of planetary dynamics prepared the door to the magic of the third order principle of spinor triality and the post-Kepplerian theorem of Alexandrov-Ovchinnikova of relativity theory via the temporal-spatial concept of ordo orbium, which still represents a treasure trove of geometry and analysis

The erroneous phenomenology of the historical Galileo causa has to be seen in the perspective of the Copernican claim to cosmic truth. Keppler had already reminded Galileo of the truth as a quality of Copernicanism in 1597, when he urgently appealed to him finally to abandon his cautious reserve and to stand up for the heliocentric world system in public. If he did so its success could no longer be in doubt. The Mathematicus Casareus or imperial mathematician had his most important experience with the power of the cosmic truth: the abandonment of the Platonic requirement that all celestial orbits be circular. This was the weightiest consession involved in 
his first spinor law of planetary motion, which the second spinor law followed by sacrifying, in the uniformity of planetary motion, the remainder of the Platonic requirement. Keppler's experience with the power of the cosmic truth was the experience of a break with a rule that was still absolutely binding for Copernicus - an experience, then, of submission to an overwhelming compulsion by astrophysical facts.

Keppler had himself published on the back of the title page to the Astronomia nova of 1609 the fact that Osiander and not Copernicus had written that unsigned preface to De revolutionibus... which categorized heliocentrism as a computational device without claim to astronomical reality. Similarly, the Roman censor exerted repressive influence onto the preface of Galileo Galilei's Dialogo sopra i due massimi sistemi del mondo tolemaico e copernicano of 1631 with the intention of circumventing the Copernican claim to cosmic truth by the prescriptions of Rome. The precriptions included the advice to circumvent the cause of the tides which was Galilei's single and supposedly strongest proof of the Copernican hypothesis.

As mentioned before, Galilei took no notice of Keppler's mathematical results in connection with the dynamical theory of planets and the influence of the moon's orbital periodicity. In lieu thereof, Galilei's phenomenology was fixed on the intuitive model that he had before him in the explanation of the phenomena of ebb and flow of the tides, and their analogy of the pendulum's motion in such a way that not only did his phenomenology fail to gain access to the rudiments of mathematical astrophysics but that access remained barred for him by his close affinity for the intuitive model. In standing closer to Goethe than to Keppler, he deprived himself of an appreciation of the brave thought-paths, that Keppler had entered on. Regardless the mistaken explanation of the oscillatory movement, the pendulum paradigm can be interpreted as an anticipated guide to Foucault's spherical pendulum device via the spin echostabilizing principle of circular inertia and the resulting kinematically relativizing action of the de Coriolis inertial force field. In this context, the term "inertia" was first introduced by Keppler in his work Epitome Astronomiae Copernicana of 16171621.

The Epitome represents a systematic textbook of heliocentrism covering Keppler's own discoveries in mathematical astrophysics [56]. Keppler's work includes an account of his second spinor law or "area law" of the ordo orbium, which had chronologically been his first, and his third spinor law $\mathrm{H}^{3}$ relating distances of the planets from the sun to their periodicities, which he discovered in 1618 shortly before he published the Epitome. These periodicities are calibrationally determined by the Witt spinor norm group $\mathrm{WS}(\mathbb{R}) \cong \mathrm{SO}(4, \mathbb{R}) / \operatorname{PSO}(1,3, \mathbb{R})$ of order 2 over the central projective collineations $\operatorname{PSO}(1,1, \mathbb{R}) \cong \mathbb{R}$. It is the Lorentz-Möbius Lie group $\operatorname{PSO}(1,3, \mathbb{R})$ which is responsible for the temporal-spatial evolution process of kinematic relativization in the complex projective manifold $\mathbb{P}_{\mathbb{C}}\left(\mathfrak{L} \mathfrak{i} \mathfrak{e}(\mathcal{N})^{*}\right)$ and the comparison between different planets of the solar system.

For more than a thousand years astronomers had been satisfied to try to predict the celestial phenomena without making claims about the actual mechanism that generated them, and many continued to be satisfied with this goal in the decades after the publication of De revolutionibus... which appeared in Nuremberg 1543. It was all that 
their tradition required of them, and it had proved to be more than sufficiently difficult to achieve by itself. Nowadays the subject of spin groups has always been inseparable from applied mathematics and quantum physics. The nuclear spin magnetic resonance tomographic analysis of the ordo orbium helps to resolve some of these dynamical difficulties by an application of quantum field theory to contact holonomy in terms of Lie group theory. In view of the Cayley-Dickson duplication strategy underlying the third order principle of spinor triality of the third Kepplerian spinor law $\mathrm{H}^{3}$ of planetary dynamics, the universal covering spinor group

$\operatorname{Spin}(4, \mathbb{R}) \cong \mathbb{S}_{3} \times \mathbb{S}_{3} \cong \operatorname{Spin}(3, \mathbb{R}) \times \operatorname{Spin}(3, \mathbb{R}) \cong \operatorname{SU}(2, \mathbb{C}) \times \operatorname{SU}(2, \mathbb{C})$

of the rotation group $\mathrm{SO}(4, \mathbb{R})$ over the "double" product Lie group $\mathrm{SO}(3, \mathbb{R}) \times$ $\mathrm{SO}(3, \mathbb{R})$ with the compact homogeneous spin manifold

$$
\mathbb{S}_{3} \cong \operatorname{SO}(4, \mathbb{R}) / \operatorname{SO}(3, \mathbb{R}) \cong \operatorname{Spin}(3, \mathbb{R}) \cong \operatorname{SU}(2, \mathbb{C}) \cong \operatorname{SL}(1, \mathbb{H}) \cong \operatorname{Aut}(\mathbb{H})
$$

emphasizes the pivotal role in the study of the dynamic quantization of the ordo orbium. The special unitary linear group $\mathrm{SU}(2, \mathbb{C})$ acts on $\mathbb{P}_{1}(\mathbb{C}) \cong \mathbb{S}_{2}$ as holomorphic and isometric transformations, and in the context of the Hopf fibration it accomodates the action of the Lie group $\mathrm{SO}(3, \mathbb{R})$ of isometries of $\mathbb{S}_{2}$. The isomorphy implies that $\mathrm{SO}(4, \mathbb{R})$ is a principal $\mathrm{SO}(3, \mathbb{R})$-bundle over the very round sphere $\mathbb{S}_{3}$. In fact, $\mathrm{SO}(4, \mathbb{R})$ is nothing else than the trivial principal bundle $\mathbb{S}_{3} \times \operatorname{SO}(3, \mathbb{R})$. As a compact Lie group, the rotation group $\mathrm{SO}(4, \mathbb{R})$ is isomorphic to the semidirect product of $\mathbb{S}_{3}$ and $\mathrm{SO}(3, \mathbb{R})$, where $\mathrm{SO}(3, \mathbb{R}) \cong \operatorname{Aut}(\mathbb{H})$ acts on the very round sphere $\mathbb{S}_{3} \cong \mathrm{SU}(2, \mathbb{C})$ in the natural way and defines, due to the Hopf principal circle bundle $\mathbb{S}_{1} \hookrightarrow \mathbb{S}_{3} \stackrel{\eta_{2}}{\longrightarrow} \mathbb{S}_{2}$ and the associated bundle epimorphism $\operatorname{Spin}(3, \mathbb{R}) \longrightarrow \operatorname{SO}(3, \mathbb{R})$, a spin structure on the tangent bundle $\mathrm{TS}_{2}$ of the round basis sphere $\mathbb{S}_{2} \cong \operatorname{SO}(3, \mathbb{R}) / \mathrm{SO}(2, \mathbb{R}) \cong \mathbb{P}_{1}(\mathbb{C})$. Theorem 10 infra will relate the submersion $\eta_{2}: \mathbb{S}_{3} \longrightarrow \mathbb{S}_{2}$ of the Hopf fibration $\mathbb{S}_{1} \hookrightarrow \mathbb{S}_{3} \stackrel{\eta_{2}}{\longrightarrow} \mathbb{S}_{2}$ with the 4dimensional real Jordan algebra $\mathfrak{h}_{2}(\mathbb{C}) \hookrightarrow \mathfrak{h}_{3}(\mathbb{C})$.

\section{The spin spectroscopic methodology of relativistic ordo orbium}

The action of the de Coriolis inertial force field, caused by the relativistic gravitation field of the spinning earth, makes the clock hand of the spin echo-stabilized, symplectic swing-plane of Foucault's spherical pendulum device rotate as time passes at a velocity depending on the latitude direction angle $\varphi \in] 0, \pi$ [ of the swivel's location where the pendulum is attached. The spatial-frequency analysis of Foucault's spherical pendulum holonomy experiment is implemented by the spectral dual pair of real Lie groups 
with mutual commutant actions along quantum channels attributed to the symmetric manifolds

$$
(\operatorname{Mp}(2, \mathbb{R}), \operatorname{PSO}(1,3, \mathbb{R})) \quad(\text { Copernicus I })
$$

with Galois group of type $(2,2)$ over the field $\mathbb{R}$ with third Galois cohomology $\mathrm{H}^{3}\left(\mathbb{R}, \mathbb{Z}_{2}\right)$ of the third order principle of spinor triality. The realignment isomorphy $\operatorname{PSO}(1,1, \mathbb{R}) \cong \mathbb{R}$ of kinematic relativization in the Minkowski time-space $\mathbb{R}^{(1,3)}$ helps to exploit the inherent spatial symmetries of the Lie group action on the compact homogeneous spin manifold $\operatorname{SO}(3, \mathbb{R}) / \operatorname{SO}(2, \mathbb{R}) \cong \mathbb{P}_{1}(\mathbb{C}) \cong \mathbb{S}_{2}$ of real dimension 2 . Due to the equivalence classes of twisted or crossed module exact sequences consisting of four-terms, the canonical mapping

$$
\mathrm{H}^{1}(\mathbb{R}, \operatorname{Spin}(3, \mathbb{R})) \longrightarrow \mathrm{H}^{3}\left(\mathbb{R}, \mathbb{Z}_{2}\right)
$$

of the first into the third Galois cohomology arises [51], where the real line will be identified with the oriented volume forms on $\Im(\mathbb{H})$ over $\mathbb{R}$. It is Galois cohomology which opens the route to the law of quadratic reciprocity by way of the SchaarLandsberg construction [46].

The map is independent of the temporal-spatial relativistic signature $(1,3)$ of the Lorentz-Möbius Lie group $\operatorname{PSO}(1,3, \mathbb{R})$. Due to the exact sequence

$$
\{1\} \longrightarrow \mathbb{Z}_{2} \longrightarrow \operatorname{Spin}(3, \mathbb{R}) \longrightarrow \operatorname{SO}(3, \mathbb{R}) \longrightarrow\{1\}
$$

it gives rise to the Galois cohomology exact sequence

$$
\begin{aligned}
& \operatorname{Spin}(3, \mathbb{R}) \longrightarrow \operatorname{SO}(3, \mathbb{R}) \longrightarrow \mathbb{R}^{\times} / \mathbb{R}^{\times 2} \\
& \longrightarrow \mathrm{H}^{1}(\mathbb{R}, \operatorname{Spin}(3, \mathbb{R})) \longrightarrow \mathrm{H}^{1}(\mathbb{R}, \operatorname{SO}(3, \mathbb{R})) \longrightarrow \mathrm{H}^{2}\left(\mathbb{R}, \mathbb{Z}_{2}\right)
\end{aligned}
$$

where the second Galois cohomology group

$$
\mathrm{H}^{2}\left(\mathbb{R}, \mathbb{Z}_{2}\right)=\{0\}
$$

of Galois group cohomology over the local field $\mathbb{R}$ is computed as the twofold Brauer group $[51,68]$

$$
\mathrm{Br}_{2}(\mathbb{R}) \hookrightarrow \operatorname{Br}(\mathbb{R})
$$

The term "Brauer group" honors Richard Dagobert Brauer (1901-1977) who made the first systematic investigation of this fundamental invariant. The only non-trivial division algebra over $\mathbb{R}$, up to isomorphism, is the four-dimensional central simple 
associative $\mathbb{R}$-algebra $\mathbb{H}$ of classical quaternions with a representation by the complex Pauli spin matrices

$$
\left\{\left(\begin{array}{ll}
1 & 0 \\
0 & 1
\end{array}\right),\left(\begin{array}{cc}
i & 0 \\
0 & -i
\end{array}\right),\left(\begin{array}{cc}
0 & 1 \\
-1 & 0
\end{array}\right),\left(\begin{array}{ll}
0 & i \\
i & 0
\end{array}\right)\right\} \hookrightarrow \operatorname{SU}(2, \mathbb{C}) .
$$

They correspond to the quaternion units which form a basis of $\mathbb{H}$ over $\mathbb{R}$. Notice that the second matrix gives rise as a traceless generator to the anaplectic group which forms a complex alternative to the metaplectic Lie group $M p(2, \mathbb{R})$ [68]. The concept of Brauer group which reads in the topologists' notation of $\mathbb{Z}_{2} \cong \mathbb{Z} / 2 \mathbb{Z}$ as

$$
\operatorname{Br}(\mathbb{R}) \cong \frac{\mathbb{Z}}{2 \mathbb{Z}} \cong \frac{1}{2} \mathbb{Z} / \mathbb{Z} \hookrightarrow \mathbb{Q} / \mathbb{Z}
$$

is able to implement the concept of quantum entanglement. The isomorphism can be thought as being an injective homomorphism $\operatorname{Br}(\mathbb{R}) \hookrightarrow \mathbb{Q} / \mathbb{Z}$ with image $\frac{1}{2} \mathbb{Z} / \mathbb{Z}$, and the twofold Brauer group $\mathrm{Br}_{2}(\mathbb{R})$ forms the spin echo kernel of multiplication by 2 within the cyclic Brauer group $\operatorname{Br}(\mathbb{R})=\mathrm{Br}_{2}(\mathbb{R})$ of order 2 .

In the context of nuclear spin magnetic resonance tomography, the spin-echo kernel acts as the identity which forms one of the two Hasse invariants of the central simple associative algebras over $\mathbb{R}$ consisting of the identity and the $\mathbb{R}$-linear involutory antiautomorphism $\varsigma: w \rightsquigarrow \bar{w}$ of $\mathbb{C}$ as the algebraic closure of the local field $\mathbb{R}$. If the groundfield is the algebraically closed field $\mathbb{C}$ of complex numbers, the only finite dimensional division algebra over $\mathbb{C} \cong \overline{\mathbb{R}}$ is $\mathbb{C}$ itself with Galois group consisting of the identity. In this case the Brauer group $\operatorname{Br}(\mathbb{C})$ reveals to be trivial $[51,68]$.

In 1977 Sir Peter Mansfield succeeded in drastically reducing the imaging times of nuclear spin magnetic resonance tomography, which at that time was typically of the order of $1 \mathrm{~h}$, by the recently discovered echo planar imaging modality to the range of a few milliseconds [47].

To illustrate the application of the spectral dual pair (Copernicus I) to the spinor approach of the classical chord-contact constrution of Poncelet's porism or closure theorem in terms of conformal involutions of a compact Riemann surface of genus one, it should be observed that the third order principle of spinor triality is able to lead to Carl Gustav Jacob Jacobi's (1804-1851) brilliant algebro-geometric discovery of 1822 of the profound relation between the existence of Poncelet's closed polygons inscribed in one smooth real conic and circumscribed about the other one generally situated in the complex projective plane $\mathbb{P}_{2}(\mathbb{C}) \hookrightarrow \mathbb{P}_{\mathbb{C}}\left(\mathfrak{L} \mathfrak{i}(\mathcal{N})^{*}\right)$, and the theory of doubly periodic elliptic functions from the vantage point of the Weierstraß $\wp$ folklore [70]. These days, the configuration of Poncelet's porism is considered as one of the most beautiful results of projective geometry [18,50].

Number theoretic prelude to the spinor triality principle Let $\mathcal{K}\left(\Lambda_{1}\right)$ denote the field of elliptic functions with respect to the period sublattice $\Lambda_{1} \hookrightarrow \mathbb{C}$ of two generators and discriminant $\Delta\left(\Lambda_{1}\right) \in \mathbb{C}$, subduced by the lattice of the compact, triality conformally triangulated, homogeneous Heisenberg nilmanifold $\Lambda \backslash \mathcal{N}$, and the constant complexvalued functions $\mathbb{C} \hookrightarrow \mathcal{K}\left(\Lambda_{1}\right)$ as a subfield. In view of the structure theorem of 
$\mathcal{K}\left(\Lambda_{1}\right)$, the isomorphic extension fields

$$
\mathcal{K}\left(\Lambda_{1}\right) \cong \mathbb{C}\left(\wp_{\Lambda_{1}}, \wp_{\Lambda_{1}}^{\prime}\right) \cong \mathbb{C}\left(\wp_{\Lambda_{1}}\right)+\mathbb{C}\left(\wp_{\Lambda_{1}}\right) \wp_{\Lambda_{1}}^{\prime}
$$

represent a three-dimensional vector space over the field $\mathbb{C}$ generated by the Weierstraß elliptic functions $\left\{\wp_{\Lambda_{1}}, \wp_{\Lambda_{1}}^{\prime}\right\}$ over the complex constants, and a two-dimensional vector space over the field $\mathbb{C}\left(\wp \Lambda_{1}\right)$ of even elliptic functions, respectively, which have a double pole at each point of the sublattice $\Lambda_{1}$, and no other poles in the complex plane $\mathbb{C}$. Thus the planar subset $\Lambda \subset \mathbb{C}$ can be characterized as the plane sublattice formed by the poles of the elliptic function $\wp_{\Lambda}[10,68,70]$.

In terms of absolutely convergent series, the Weierstraß triality representations

$$
\wp_{\Lambda_{1}}(w)=\frac{1}{w^{2}}+\sum_{v \in \Lambda_{1}-\{0\}}\left(\frac{1}{(w-v)^{2}}-\frac{1}{v^{2}}\right), \wp_{\Lambda_{1}}^{\prime}(w)=-2 \sum_{v \in \Lambda_{1}} \frac{1}{(w-v)^{3}}
$$

of double and triple poles, respectively, hold true. Clearly, the elliptic function $\wp_{\Lambda_{1}}^{\prime}$ : $w \rightsquigarrow \frac{\partial}{\partial w} \wp_{\Lambda_{1}}(w)$ as the derivative of $w \rightsquigarrow \wp_{\Lambda_{1}}(w)$ represents an odd meromorphic function with poles in $\Lambda_{1} \hookrightarrow \mathbb{C}$, so that the identities

$$
\wp_{\Lambda_{1}} \cdot \wp_{\Lambda_{1}}^{\prime}=\frac{1}{12} \wp_{\Lambda_{1}}^{\prime \prime \prime}, \quad \wp_{\Lambda_{1}}^{2}=\frac{1}{6} \wp_{\Lambda_{1}}^{\prime \prime}+5 \sum_{v \in \Lambda_{1}-\{0\}} \frac{1}{v^{4}}
$$

hold. These identities were already known to Ferdinand Gotthold Max Eisenstein in 1847, independently of Jacobi's Fundamenta Nova Theorice Functionum Ellipticarum of 1829. Eisenstein was aware of the concept of absolute convergence, but he was unaware of the concept of local uniform convergence [70]. For infinite series which are not absolutely convergent, he replaced the aforementioned summation process $\sum_{\Lambda_{1}-\{0\}}$ by the symbol $\sum_{e}^{\prime}$ to lay the foundations of elliptic functions based upon entirely original ideas without recourse to the function-theoretic innovations due to Karl Theodor Wilhelm Weierstraß (1815-1897) who ignored the Eisenstein calculus by non compelling reasons. The theta function defined by the Weierstraß product

$$
\sigma_{\Lambda_{1}}(w)=w \prod_{v \in \Lambda_{1}-\{0\}}\left(1-\frac{w}{v}\right) \cdot e^{\left[\frac{w}{v}+\frac{1}{2}\left(\frac{w}{v}\right)^{2}\right]}
$$

where $w \in \mathbb{C}$, represents an entire holomorphic function which is odd on the metaplectic coadjoint orbit $\mathcal{O}_{1} \cong \mathbb{C}$ within the real dual vector space $\mathfrak{L} \mathfrak{i}(\mathcal{N})^{*}$, and has zeros of order one at all lattice points. The Weierstraß sigma function with respect to the period sublattice $\Lambda_{1} \hookrightarrow \mathbb{C}$ satisfies the equation of degree one homogeneity

$$
\sigma_{\lambda \Lambda_{1}}(\lambda w)=\lambda \sigma_{\Lambda_{1}}(w)
$$


and the equation

$$
\wp \Lambda_{1}(v)-\wp \Lambda_{1}(w)=-\frac{\sigma_{\Lambda_{1}}(v+w)-\sigma_{\Lambda_{1}}(v-w)}{\sigma_{\Lambda_{1}}^{2}(v) . \sigma_{\Lambda_{1}}^{2}(w)}
$$

for points $v, w \in \mathbb{C}-\Lambda_{1}$. The limit $v \longrightarrow w$ yields

$$
\wp_{\Lambda_{1}}^{\prime}(w)=-\frac{\sigma_{\Lambda_{1}}(2 w)}{\sigma_{\Lambda_{1}}(w)^{4}}
$$

where $\lambda \in \mathbb{C}^{\times}$. Using Jacobi's Fundamenta notation, let

$$
q=e^{2 \pi i \tau} \quad(\Im \tau>0)
$$

where $\tau \in \mathbb{C}$ belongs to the open upper complex plane. The infinite product

$$
P(q)=\prod_{\nu \geq 1}\left(1-q^{\nu}\right)
$$

has been used by Jacobi to establish the theta series

$$
\frac{1}{2} \sum_{n \in \mathbb{Z}}(-1)^{n}(2 n+1) q^{\frac{n^{2}+n}{2}}=P(q)^{3}
$$

and

$$
\sum_{n \in \mathbb{Z}}(-1)^{n+1} q^{\frac{(n+1)(3 n+2)}{6}}=P\left(q^{\frac{1}{3}}\right)
$$

which are also results of the Eisenstein calculus. Poisson summation on the compact homogeneous Heisenberg nilmanifold $\mathbb{Z}^{3} \backslash \mathcal{N}$ gives rise to the theta expressions

$$
T_{0}=\frac{1}{2} \sum_{n \in \mathbb{Z}} q^{\frac{n^{2}+n}{2}}=P(q) \prod_{\nu \geq 1}\left(1+q^{\nu}\right)^{2}
$$

by the Fourier transform approach to theta functions, and

$$
\begin{aligned}
& T_{1}=\sum_{n \in \mathbb{Z}}(-1)^{n} q^{\frac{n^{2}}{2}}=P(q) \prod_{\nu \geq 0}\left(1-q^{\nu+\frac{1}{2}}\right)^{2}, \\
& T_{2}=\sum_{n \in \mathbb{Z}} q^{\frac{n^{2}}{2}}=P(q) \prod_{\nu \geq 0}\left(1+q^{\nu+\frac{1}{2}}\right)^{2} .
\end{aligned}
$$


Thus the triple product

$$
\prod_{0 \leq \varkappa \leq 2} T_{\varkappa}=P(q)^{3}
$$

follows. The material on theta functions is fundamental in algebraic functions and number theory.

Eisenstein succeeded in supplying new identities even for the elementary trigonometric functions by starting with the infinite series of Bernoulli numbers

$$
\pi \cot \pi x=\frac{1}{x}+\sum_{m \geq 1}\left(\frac{1}{x+m}+\frac{1}{x-m}\right)=\frac{1}{x}-\sum_{m \geq 1}(2 \pi)^{2 m} B_{m} \frac{x^{2 m-1}}{(2 m) !}
$$

with $x \in \mathbb{R}-\mathbb{Z}$. Expressing chord-contact geometrically the cosecant csc $x$ by means of the logarithmic derivative of $x \rightsquigarrow \sin \frac{x}{2}$ along the one-dimensional center $\mathcal{Z} \hookrightarrow \mathcal{N}$ yields

$$
\csc x=\frac{1}{2} \cot \frac{x}{2}-\frac{1}{2} \cot \frac{x+\pi}{2}
$$

and as a consequence of the Eisenstein identity

$$
\pi \csc \pi x=\frac{1}{x}+\sum_{e}^{\prime} \frac{(-1)^{m}}{x+m}
$$

the sinus cardinalis

$$
\operatorname{sinc} x=\prod_{e}^{\prime}\left(1+\frac{x}{m}\right) \text {. }
$$

The classical infinite product, originally discovered by Jacobi's idol Leonhard Euler (1707-1783), represents the convolution kernel of the Nyquist-Shannon sampling theorem of bandlimited signal processing and slice-selective spin excitation, saturation and refocusing in nuclear spin magnetic resonance tomography. A sinc pulse consists of several adjacent lobes of alternating polarity. The central lobe has the highest amplitude and is also twice as wide as every other lobe. On commercial nuclear spin magnetic resonance scanners, sinc pulses, especially symmetric ones, serve for spin excitation purposes with small flip angles [9].

The totality of all entire holomorphic functions of exponential type at most $\pi$ that are square integrable along the real axis $\mathbb{R}$ represents the Paley-Wiener space over $\mathbb{C}[20]$. Since the Fourier transform defines an isometry, the Paley-Wiener space is a complex Hilbert space, isometrically isomorphic to $L^{2}([-1,+1])$. Its reproducing kernel, given by the function

$$
\mathbb{C} \times \mathbb{C} \ni(w, v) \rightsquigarrow \operatorname{sinc}(w-\bar{v}),
$$


is uniquely determined and gives rise to the cardinal orthonormal basis

$$
(w \rightsquigarrow \operatorname{sinc}(w-m))_{m \in \mathbb{Z}}
$$

of the digitizing bandlimited signals.

Beyond the construction of elementary trigonometric and elliptic functions, Eisenstein was able to prove the laws of cubic and biquadratic reciprocity as well by replacing the trigonometric functions by certain elliptic functions. He prepared the route to Leopold Kronecker's (1823-1891) class field theoretical results as a number-theoretic allegro con brio [70], and also the route to the work of Kronecker's student Kurt Hensel (18611941). One of Kronecker's propositions was Mathesis et ars et scientia dicenda. In his work, he made frequent use of the Eisenstein procedures $\sum_{e}^{\prime}$ and $\prod_{e}^{\prime}$, but he had available at his disposal also the powerful Poisson summation procedure to establish his famous limit formula in terms of the function

$$
s \rightsquigarrow-1-\frac{s}{12} \log \left(\Delta\left(\Lambda_{1}\right) \bar{\Delta}\left(\Lambda_{1}\right)\right) .
$$

The limit formula depends only upon the period sublattice $\Lambda_{1} \hookrightarrow \mathbb{C}$ of the basic elliptic functions $\left\{\wp_{\Lambda_{1}}, \wp_{\Lambda_{1}}^{\prime}\right\} \hookrightarrow \mathcal{K}\left(\Lambda_{1}\right)$ and not upon the choice of the generators of the sublattice $\Lambda_{1} \hookrightarrow \Lambda$. In the light of the theory of tempered distributions it gives rise to the summatory processes of pulse sequences and for imaginary values of the argument to the Bessel functions. The Poisson summation procedure actually forms a major tool in the areas of nuclear spin magnetic resonance tomography and classical number theory.

The close relationship to the third order principle of spinor triality for a field $K$ with Galois group of type $(2,2)$ over the field $\mathbb{R}$ is a surprising aspect of the underlying half-spinor concept with strong influence on the deep number theoretic work of Johann Peter Gustav Lejeune Dirichlet (1805-1859) concerning the zeta-function $\zeta_{K}$ of $K$ and the zeta-function $\zeta=\zeta_{\mathbb{Q}}$ over the field of rational numbers $\mathbb{Q}$ and the triple of quadratic subfields $\left(k_{\varkappa}\right)_{0 \leq \varkappa \leq 2}$ of $K$ which is the compositum of any two of them in the product

$$
\zeta_{K}=\zeta \prod_{0 \leq \varkappa \leq 2} L_{\varkappa}
$$

of the associated Abelian Dirichlet $L$-functions

$$
L_{\varkappa}=\frac{\zeta \varkappa}{\zeta} \quad(0 \leq \varkappa \leq 2)
$$

over $\mathbb{Q}$ [70], and $k_{0}=\mathbb{Q}(i)$ denotes the Gaussian algebraic number field. The Abelian Dirichlet $L$-functions actually are special cases of the Hecke $L$-functions that seem to occur naturally in aithmetic.

Kronecker's limit formula permits to express the orders $\left(h_{\varkappa}\right)_{0 \leq \varkappa \leq 2}$ of the ideal class groups of $\left(k_{\varkappa}\right)_{0 \leq \varkappa \leq 2}$, and to express the product $\prod_{0 \leq \varkappa \leq 2}$ in terms of elliptic 
functions. In the context of basic local class field theory, Eisenstein summatory procedure emphasizes directly the secret link between the algebraic theory of spinors and Clifford algebras as developed by the great algebrist Claude Chevalley (1909-1984). He introduced the concept of locally compact topological group of idèles in order to complete the Legendre-Hilbert-Artin tower and use infinite Galois extensions for the formulation of the essential ideas of the algebraic aspects of class field theory [70].

To summarize the number theoretic prelude to the third order principle of spinor triality, the heart of class field theory is the description of the Abelian extensions of imaginary quadratic fields $k$ in terms of special values of elliptic modular functions and to extend this to the study of all finite Abelian extensions $K$ of an arbitrary number field, which are in correspondence to certain subgroups of the idèle group whose quotient groups are realizations of the Galois group of $K$ over the number field by virtue of the isomorphy theorem of class field theory $([17,68])$.

For two equivalent sublattices $\Lambda_{1}$ and $\Lambda_{1}^{\prime}=\lambda \Lambda_{1}$ of the complex plane $\mathbb{C}$, the following relations of double and triple homogeneity degree, respectively, hold:

$$
\wp_{\Lambda_{1}}(w)=\lambda^{2} \wp_{\lambda \Lambda_{1}}(\lambda w), \quad \wp_{\Lambda_{1}}^{\prime}(w)=\lambda^{3} \wp_{\lambda \Lambda_{1}}^{\prime}(\lambda w)
$$

The Weierstraß elliptic function $\wp_{\Lambda_{1}}$ admits with exception of the lattice points no other periods. The affine $\mathbb{C}$-basis

$$
\left\{1, \wp_{\Lambda_{1}}, \wp_{\Lambda_{1}}^{\prime}\right\}
$$

of the complex vector space consisting of the complex constants and the meromorphic functions of order two and three, respectively, with respect to the period sublattice $\Lambda_{1} \hookrightarrow \mathbb{C}$ subduced by the lattice $\Lambda$ of the compact, triality conformally triangulated, homogeneous Heisenberg nilmanilfold $\Lambda \backslash \mathcal{N}$ gives rise to the projection

$$
E(\mathbb{C}) \longrightarrow \mathbb{P}_{2}(\mathbb{C}) \hookrightarrow \mathbb{P}_{\mathbb{C}}\left(\mathfrak{L i} \mathfrak{i}(\mathcal{N})^{*}\right)
$$

of the non-singular elliptic curve of degree three over the complex numbers by generating a plane cubic curve as a projective model of $E(\mathbb{C})$ of genus one with foliation

$$
\mathbb{P}_{\mathbb{H}}\left(\mathrm{T}_{\bullet}\left(\mathbb{S}_{4}\right)\right) \hookrightarrow \mathbb{P}_{\mathbb{H}}\left(\mathfrak{L} \mathfrak{i} \mathfrak{e}(\mathcal{N})^{*}\right)
$$

where $\mathbb{S}_{4} \cong \mathbb{P}_{1}(\mathbb{H})$, and octonionic extension

$$
\mathbb{P}_{\mathbb{O}}\left(\mathrm{T}_{\bullet}\left(\mathbb{S}_{8}\right)\right) \hookrightarrow \mathbb{P}_{\mathbb{O}}\left(\mathfrak{L} \mathfrak{i e}(\mathcal{N})^{*}\right)
$$

where $\mathbb{S}_{8} \cong \mathbb{P}_{1}(\mathbb{O})$. The spectral dual pair (Copernicus I) is deep enough to lead by the construction of the great geometer Jean-Victor Poncelet (1788-1867) to the commutative field $\mathcal{K}\left(\Lambda_{1}\right)$ of elliptic functions and the collinearity condition of the 
chord-contact configuration

$$
\operatorname{det}\left(\begin{array}{ccc}
1 & \wp \Lambda_{1}(v+w)-\wp_{\Lambda_{1}}^{\prime}(v+w) \\
1 & \wp_{\Lambda_{1}}(w) & \wp_{\Lambda_{1}}^{\prime}(w) \\
1 & \wp_{\Lambda_{1}}(v) & \wp_{\Lambda_{1}}^{\prime}(v)
\end{array}\right)=0
$$

allows to parametrize the canonical line bundle of $\mathbb{C}$ by means of the linear gradient

$$
\frac{\wp_{\Lambda_{1}}^{\prime}(v)-\wp_{\Lambda_{1}}^{\prime}(w)}{\wp_{\Lambda_{1}}(v)-\wp_{\Lambda_{1}}(w)}
$$

Thus in terms of the automorphism of the incidence correspondence which consists of the composition of the pair of conformal involutions corresponding to the even or respectively odd pure spinors of spinor triality theory [10], the collinearity condition gives rise to a translation with respect to the Abelian chord-contact law of the nonsingular elliptic curve $E(\mathbb{C})$ and the Dehn twist of the quotient torus $\mathbb{C} / \Lambda_{1}$ in order to establish algebro-geometrically the Poncelet porism by the marked fixed point $O \in \mathbb{C}$ of the translation.

Expressing $\wp_{\Lambda_{1}}(v+w)=\wp_{\Lambda_{1}}(-v-w)$ and $-\wp_{\Lambda_{1}}^{\prime}(v+w)=\wp_{\Lambda_{1}}^{\prime}(-v-w)$ rationally in terms of the quadruple $\left\{\wp_{\Lambda_{1}}(v), \wp_{\Lambda_{1}}(w), \wp_{\Lambda_{1}}^{\prime}(v), \wp_{\Lambda_{1}}^{\prime}(w)\right\}$, the collinearity condition of the chord-contact configuration implies the quadratic addition formula

$$
\wp_{\Lambda_{1}}(v+w)=\frac{1}{4}\left[\frac{\wp_{\Lambda_{1}}^{\prime}(v)-\wp_{\Lambda_{1}}^{\prime}(w)}{\wp_{\Lambda_{1}}(v)-\wp_{\Lambda_{1}}(w)}\right]^{2}-\left(\wp_{\Lambda_{1}}(v)+\wp_{\Lambda_{1}}(w)\right)
$$

for arguments $v \in \mathbb{C}$ and $w \in \mathbb{C}$, where the complex numbers $v+w, v-w, v$ and $w$ do not belong to the period sublattice $\Lambda_{1} \hookrightarrow \mathbb{C}$. The obvious limiting process

$$
\lim _{v \longrightarrow w} \frac{\wp_{\Lambda_{1}}^{\prime}(v)-\wp_{\Lambda_{1}}^{\prime}(w)}{\wp \Lambda_{1}(v)-\wp_{\Lambda_{1}}(w)}=\frac{\wp^{\prime \prime} \Lambda_{1}(w)}{\wp_{\Lambda_{1}}^{\prime}(w)}
$$

provides the quadratic duplication formula associated with (Copernicus I)

$$
\wp_{\Lambda_{1}}(2 w)=\frac{1}{4}\left[\frac{\wp_{\Lambda_{1}}^{\prime \prime}(w)}{\wp_{\Lambda_{1}}(w)}\right]^{2}-2 \wp_{\Lambda_{1}}(w)
$$

where $w \in \mathbb{C}$ does not belong to the period sublattice $\Lambda_{1}$ [45]. The projective model of the non-singular cubic curve

$$
E(\mathbb{C}) \cong \mathbb{C} / \Lambda_{1}
$$


as a conformally represented Riemann surface of finite ramification points $\{\alpha, \beta, \gamma\} \hookrightarrow$ $\mathbb{C}$ given by the plane cubic curve with affine equation

$$
y^{2}=(x-\alpha)(x-\beta)(x-\gamma)
$$

and exactly one point $\infty \in \mathbb{P}_{2}(\mathbb{C})$ at infinity makes sure via the third order principle of spinor triality that for a point $\left(x^{\prime}, y^{\prime}\right) \in E(\mathbb{C})$ there exists $(x, y) \in E(\mathbb{C})$ with

$$
2(x, y)=\left(x^{\prime}, y^{\prime}\right)
$$

if and only if the linear terms $\left\{x^{\prime}-\alpha, x^{\prime}-\beta, x^{\prime}-\gamma\right\}$ of $y^{2}$ are squares. In this case, the group $E(\mathbb{C})$ under its Abelian group law is 2 divisible, the multiplication by 2 is a group endomorphism $E(\mathbb{C}) \longrightarrow E(\mathbb{C})$ so that the poimts of order two on the complex quotient torus $\mathbb{C} / \Lambda_{1}$ form the non-cyclic Klein four-group $\mathfrak{V}_{4}$ of o. For a natural number $N$ the Poncelet construction gives rise to a closed polygon of $N$ sides with arbitrary initial data by the group of $N$-division points on the complex quotient torus $E(\mathbb{C})$ of finite order $N^{2}$. It represents the direct sum of two cyclic groups of order $N$ and forms the kernel of the isogeny $N: E(\mathbb{C}) \longrightarrow E(\mathbb{C})$.

The Lorentz-Möbius Lie group $\operatorname{PSO}(1,3, \mathbb{R})$ represents the isometry group of the one-sheeted unit hyperboloid, known as the three-dimensional de Sitter space. The metaplectic Lie group $\operatorname{Mp}(2, \mathbb{R})$ involves the quantum field twisting $\mathbb{Z}_{2}$ [69], whereas the Lie algebra $\mathfrak{L} \mathfrak{i} e(\operatorname{SO}(1,3, \mathbb{R}))$ is spanned over $\mathbb{R}$ by the linear differential operators

$$
\left\{t \frac{\partial}{\partial x_{j}}+x_{j} \frac{\partial}{\partial t}, t \frac{\partial}{\partial x_{j}}-x_{j} \frac{\partial}{\partial t} \mid 1 \leq j \leq 3\right\}
$$

It follows the basic fact that the $\mathfrak{L} \mathfrak{i} e(\operatorname{PSO}(1,3, \mathbb{R}))$ action commutes with the $\mathfrak{L} \mathfrak{i} e(\operatorname{SL}(2, \mathbb{R}))$ and the $\mathfrak{L} \mathfrak{i} \mathfrak{( M p}(2, \mathbb{R}))$ action on the Schwartz space $\mathcal{S}_{\mathbb{C}}\left(\mathbb{R}^{4}\right)$, so that the spectral decomposition of one of them completely determines the spectral decomposition of the other one.

Theorem 2 The spectral dual pair (Copernicus I) along attributed quantum channels determines uniquely up to a common factor the coefficients of any real projective quadric of $1 \leq$ rank $\leq 4$ as eigenvalues of eigenspaces of an endomorphism of the four-dimensional vector space $\mathbb{R}^{(1,3)}$, independently of the Cartesian frame of the relativistic dynamics inherent to the Minkowski time-space $\mathbb{R}^{(1,3)}$.

The uniqueness of the spectral decomposition is utilized in the post-Kepplerian proof of a relativistic characterization of lightlike dynamics in terms of projective collineations of the axial future/past double light-ray cone which forms a real projective quadric characterized by rank three and rank three on the absolute hyperplane $\mathfrak{L} \mathfrak{i}(\mathcal{N})$ * of the four-dimensional Minkowski time-space $\mathbb{R}^{(1,3)}$ with real generators in terms of the associated tomographic affinization.

The affine tomographic platform $\mathbb{P}_{\mathbb{C}}\left(\mathfrak{L i} \mathfrak{i}(\mathcal{N})^{*}\right)$. The affine Lie subgroup $\operatorname{AGL}(3, \mathbb{R})$ of the Lorentz-Möbius Lie group $\operatorname{PSO}(1,3, \mathbb{R})$ is isomorphic to the semi-direct product of $\mathbb{R}^{2}$ and $\mathbb{R}_{+}^{\times}$, namely the classical group of parallel translations and dilations of 
the real plane $\mathbb{R}^{2}$ according to

$$
\operatorname{AGL}(3, \mathbb{R}) \cong(\mathbb{R} \oplus \mathbb{R}) \rtimes \mathbb{R}_{+}^{\times} \hookrightarrow \operatorname{PSO}(1,3, \mathbb{R})
$$

In terms of triangular block matrices, the isomorphy

$$
\operatorname{AGL}(3, \mathbb{R}) \cong\left\{\left(\begin{array}{cc}
1 & 0 \\
\mathfrak{x} & \left(\begin{array}{ll}
y & 0 \\
0 & y
\end{array}\right)
\end{array}\right) \in \operatorname{GL}(3, \mathbb{R}) \mid \mathfrak{x} \in \mathbb{R} \oplus \mathbb{R}, y>0\right\}
$$

arises. The Iwasawa decomposition of $\operatorname{PSO}(1,3, \mathbb{R})$ demonstrates that any element of the affine group $\operatorname{AGL}(3, \mathbb{R})$ represents in a unique way the product of an element of $\operatorname{PSO}(1,3, \mathbb{R})$ and a rotation in the compact Lie group $\mathrm{SO}(3, \mathbb{R})$. The covering epimorphism

$$
\operatorname{Spin}(3, \mathbb{R}) \longrightarrow \operatorname{SO}(3, \mathbb{R})
$$

leads to the global third order principle of spinor triality which offers an elegant proof for a relativistic characterization of the simple Lorentz-Möbius Lie group $\operatorname{PSO}(1,3, \mathbb{R})$ of isometries of the four-dimensional Minkowski time-space $\mathbb{R}^{(1,3)}$ in terms of the fundamental theorem of Alexandrov-Ovchinnikova of relativity theory or its analogous theorem for the Laguerre transformation. The proof of this "characterization of geometrical mappings under mild hypotheses" proceeds according to the geometric hierarchy of Felix Klein's famous "Erlanger Programm" of 1872 by the gradual transition from affine geometry to metric geometry in terms of the relevant projective geometry. It is known that the transition to the metric geometry is performed by the (4 - 2)-dimensional absolute quadric $\mathbb{S}_{2}^{\frac{1}{2}}$ of sphere radius $\frac{1}{2}$ within the three-dimensional hyperplane at infinity $\mathfrak{L} \mathfrak{i} \mathfrak{e}(\mathcal{N}) *$ of the four-dimensional Minkowski time-space $\mathbb{R}^{(1,3)}$. The direct transition of an affine space of dimension $\geq 2$ to the projective space transfers dilations and parallel translations onto central projective collineations, which keep fixed the absolute points and where translations adhere to their centers at infinity. If the assignment

$$
f: \mathbb{R}^{(1,3)} \longrightarrow \mathbb{R}^{(1,3)}
$$

denotes a non-identical bijectve mapping which does not necessarily need to be linear nor continuous and transforms the axial future/past double light-ray cone onto the corresponding affine double light-ray cone within the tomographic affinization of the Minkowski time-space $\mathbb{R}^{(1,3)}$, then the bijection $f \neq$ id of lightlike vectors reveals to be a multiple of an isometry in $\operatorname{PSO}(1,3, \mathbb{R})$ modulo a translation of $\mathbb{R}^{(1,3)}[1,2]$. The proof depends on the fact that a pair of parallel two-dimensional affine planes, or equivalently a real projective quadric of rank two $L \hookrightarrow \mathbb{R}^{(1,3)}$ and minimal rank one on the two-dimensional affine plane at infinity, which intersects the affine double lightray cone of the Minkowski time-space $\mathbb{R}^{(1,3)}$ in two generating lines, and both tangent to the absolute Riemann contact sphere $\mathbb{S}_{2}^{\frac{1}{2}} \hookrightarrow \mathbb{P}_{\mathbb{C}}\left(\mathfrak{L} \mathfrak{i} \mathfrak{e}(\mathcal{N})^{*}\right)$, admits as its image 
$f(L) \hookrightarrow \mathbb{R}^{(1,3)}$ a pair of intersecting affine two-dimensional planes, or equivalently a real projective quadric of rank two inside the affine vector space $\mathbb{R}^{(1,3)}$ and a real projective quadric of rank two on the two-dimensional affine plane at infinity, in conjunction with a real projective quadric with one positive eigenvalue and a real projective quadric with one positive eigenvalue at the two-dimensional affine plane at infinity. The subtending generator lines give rise to photon counting half-angles [40]. The intersection $L \cap f(L) \cong \operatorname{PSO}(1,1, \mathbb{R})$ can be considered as the axis $\ell$ of a central projective collineation of the $(2+1)$-dimensional affine space $\mathfrak{L i e}(\mathcal{N})^{*}$. As a real projective quadric of rank two, the flat spectrum $L$ in conjunction with the flat spectrum $f(L)$ closes for three photons the lightlike irreducible Cayley-Feynman 2complex of the dihedral group $\mathcal{D}_{3}$ of order 6 , as displayed in Theorem 3 infra. It is known that the preservation of collinearity of triples of collinear points is sufficient for the affinization procedure. Gluing the ruled tomographic slices by the projective collineations

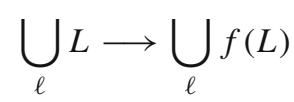

permits to turn the bijection $f$ into an affine isometry of the Minkowski time-space $\mathbb{R}^{(1,3)}$ modulo a dilation along the axis $\ell \cong \operatorname{PSO}(1,1, \mathbb{R})$. The tomographic affinization is completed by means of the polarization of the indefinite quadratic form of temporalspatial relativistic signature $(1,3)$ associated with the double light-ray cone of lightlike vectors at the origin of the four-dimensional vector space $\mathbb{R}^{(1,3)}$.

Remark 1 The contact geometry of the metaplectic coadjoint orbit model of the unitary dual $\widehat{\mathcal{N}}$ will make apparent that the tomographic affinization of the post-Kepplerian theorem of Alexandrov-Ovchinnekova by means of the axis $\ell \cong \operatorname{PSO}(1,1, \mathbb{R})$ of central projective collineations exhibits the conditions

$$
\operatorname{dim}_{\mathbb{R}} \mathfrak{L i e}(\mathcal{N})^{*}=3, \quad \operatorname{dim}_{\mathbb{R}} \mathbb{R}^{(1,3)}=4
$$

as being metaplectically appropriate. There is a collection of several counterexamples elaborated in the literature which are valid for the planar case. The same restriction of dimension is implied by the Laguerre contact geometric approach in terms of oriented spears and the contact spheres of the axial future/past double light-ray cone of lightlike vectors acting as Laguerre phase cycles. The constancy of speed of light which is basic for the principal $\operatorname{PSO}(1,3, \mathbb{R})$-Lorentz frame bundle reveals the mapping $f$ as a Laguerre contact geometric transformation of Minkowski time-space $\mathbb{R}^{(1,3)}$ corresponding the Lorentz-Möbius transformation of relativity theory.

The Blaschke-Grünwald model of Laguerre contact geometry (Fig. 3), well known from Computer Aided Geometric Design (CAGD), defines the spears as the points of a circular cylinder $\mathfrak{Z} \hookrightarrow \mathfrak{L i e}(\mathcal{N})^{*}$ and axis $\mathfrak{L i e}(\mathcal{Z})^{*}$. The Laguerre cycles are defined as the intersections of the two-dimensional planes with $\mathfrak{Z}$ which are not parallel to the axis of $\mathcal{Z}$, and hence transversal to the covariant pages of the open-book foliation in $\mathbb{P}_{\mathbb{C}}\left(\mathfrak{L i e}(\mathcal{N})^{*}\right)$ with $\mathbb{S}_{1} \cong \mathbb{T}$-binding. A spear or ray tangent is called to be in contact with a Laguere cycle if an only if the cycle is located on the intersecting 


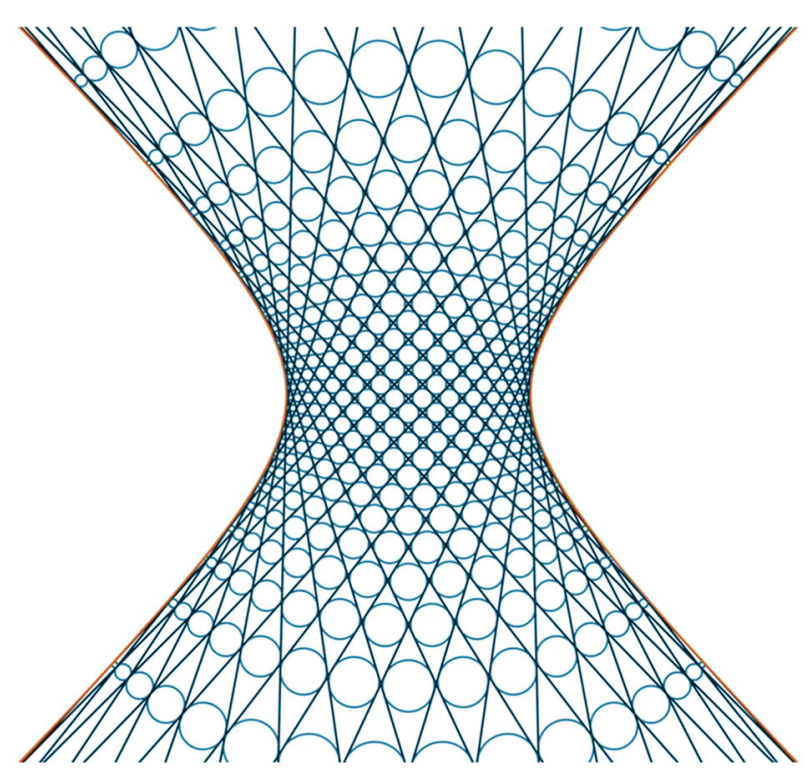

Fig. 3 Global-local duality: Laguerre contact geometry of the three-dimensional de-Sitter-space admitting the Lorentz-Möbius Lie group $\operatorname{PSO}(1,3, \mathbb{R})$ as its isometry group. The one-sheeted unit hyperboloid represents a real projective quadric of rank four inside the four-dimensional affine vector space $\mathbb{R}^{(1,3)}$ and a real projective quadric of rank three on the two-dimensional affine plane at infinity, in conjunction with a real projective quadric with two positive eigenvalues, and a real projective quadric with two positive eigenvalues at the two-dimensional affine plane at infinity

plane. A Laguerre contact geometric transformation of Minkowski time-space $\mathbb{R}^{(1,3)}$ corresponding the Lorentz-Möbius transformation of relativity theory represents a bijection of the set of cycles.

Remark 2 The ensuing Laguerre contact geometric formulation of the theorem of Alexandrov-Ovchinnikova in conjunction with the spectral duality (Copernicus I) along attributed quantum channels opens the avenue to spintronics, the experimental science of projective measurement of spinor based information transport and processing with electrons and photons [31].

An important application of the the spectral dual pair of real Lie groups (Copernicus I) is the three-dimensional extension of the dynamics of Poncelet's porism of algebraic geometry. It turns up a class of closed polyhedra in the complex projective manifold $\mathbb{P}_{\mathbb{C}}\left(\mathfrak{L} \mathfrak{i}(\mathcal{N})^{*}\right)$ which are both inscribed in and circumscribed about a pair of smooth doubly ruled quadric surfaces in general position. These configurations are even more symmetric than those arising from the explicit solution of the classical Poncelet construction in the complex plane which concerns closed polygons simultaneously inscribed in one smooth conic and circumscribed about another. Following the lead of Jacobi, it is known that the proof involves the familiar but profound fact that the elliptic curves over $\mathbb{C}$ may be uniformized so as to have a flat Riemannian metrics. Actually, the Poncelet construction and the addition theorem for non-singular elliptic functions 


$$
E(\mathbb{C}) \hookrightarrow \mathbb{P}_{1}(\mathbb{C}) \times \mathbb{P}_{1}(\mathbb{C}) \longrightarrow \mathbb{P}_{2}(\mathbb{C}) \hookrightarrow \mathbb{P}_{\mathbb{C}}\left(\mathfrak{L i e}(\mathcal{N})^{*}\right)
$$

are essentially equivalent. Indeed, in terms of Laguerre contact geometry the basic variety underlying the Poncelet construction is the incidence correspondence $E(\mathbb{C})$ of spears or ray tangents and Laguerre cycles. It represents an algebraic surface of Euler characteristic zero and genus one which can be represented by the two-sheeted covering of the Riemann sphere $\mathbb{P}_{1}(\mathbb{C}) \cong \mathbb{S}_{2} \cong \mathrm{SO}(3, \mathbb{R}) / \mathrm{SO}(2, \mathbb{R})$ so that the birational isomorphy of the quotient torus $E(\mathbb{C}) \cong \mathbb{C} / \Lambda_{1}$ holds in terms of the period sublattice $\Lambda_{1} \hookrightarrow \mathbb{C}$ of the basic theorem of the classical Abel-Jacobi theory [18]. The associated mapping

$$
E(\mathbb{C}) \longrightarrow \mathbb{C} / \Lambda_{1}
$$

represents a biholomorphic isomorphism of non-singular Riemann surfaces.

The third order principle of spinor triality via the spectral flattening procedure of the Alexandrov-Ovchinnikova theorem shows how again the three-dimensional configurations may be constructed analytically from elementary properties of the commutative group law on an elliptic curve with a marked rational point $O$ chosen as the identity of height 1 for the group law obtained from the additive structure of the complex plane $\mathbb{C}$ on which the lattice $\Lambda_{1}$ is projected. The marked origin $O$ corresponds to the point $\infty \in \mathbb{P}_{2}(\mathbb{C})$. The Poncelet properties reveal the isomorphism of the smooth doubly ruled quadric surfaces to the complex manifold $\mathbb{P}_{1}(\mathbb{C}) \times \mathbb{P}_{1}(\mathbb{C})$ which induces on the real projective space $\mathbb{P}\left(\mathfrak{L i e}(\mathcal{N})^{*}\right)$ a hyperbolic projective quadric $\Psi$ of rank four inside the four-dimensional affine vector space $\mathbb{R}^{(1,3)}$ and a real projective quadric of rank three on the two-dimensional affine plane at infinity, in conjunction with a real projective quadric with two positive eigenvalues, and a real projective quadric with two positive eigenvalues at the two-dimensional affine plane at infinity. For such a quadric and any straight line $L \hookrightarrow \mathbb{P}\left(\mathfrak{L i e}(\mathcal{N})^{*}\right)$ there will be two tangent planes to $\Psi \hookrightarrow \mathbb{P}\left(\mathfrak{L i e}(\mathcal{N})^{*}\right)$ containing $L$ if and only if $L$ meets $\Psi$. Thus if $\Psi \hookrightarrow \mathbb{P}\left(\mathfrak{L i e}(\mathcal{N})^{*}\right)$ and $\Psi^{\prime} \hookrightarrow \mathbb{P}\left(\mathfrak{L i e}(\mathcal{N})^{*}\right)$ are both hyperbolic quadrics and meet in a curve, so that every line lying on $\Psi$ meets $\Psi^{\prime}$ and vice versa, Poncelet's construction carried out in $\mathbb{P}\left(\mathfrak{L i e}(\mathcal{N})^{*}\right)$ generates a configuration of real bitangent planes in $\mathbb{P}\left(\mathfrak{L i e}(\mathcal{N})^{*}\right)$ which forms a real polyhedron in $\mathbb{P}\left(\mathfrak{L i e}(\mathcal{N})^{*}\right)$ simultaneously inscribed in and circumscribed about $\Psi$ and $\Psi^{\prime}$. The Euler characteristic of the polyhedron is zero so that the polyhedron represents a Picard torus in the three-dimensional real vector space $\mathfrak{L i e}(\mathcal{N})^{*}$.

A direct computation of the harmonic analysis of the symplectic swing-plane of Foucault's spherical pendulum device implies the central identity for the preservation of the attached Casimir invariants or squared orbital angular momentum operators

$$
\Omega_{(2,2)}=\Omega_{(1,3)} \quad \text { (Casimir) }
$$

in the centers of the universal enveloping Lie algebras $\mathfrak{Z} \mathfrak{U}(\mathfrak{L i e}(\operatorname{Mp}(2, \mathbb{C})))$ and $\mathfrak{Z U}(\mathfrak{L i v}(\mathrm{SO}(1,3, \mathbb{C})))$, respectively [41]. It follows for the inherent finite Coxeter symmetry: 
Theorem 3 The irreducible spherical diagram underlying the identity (Casimir) is given by the lightlike irreducible Cayley-Feynman 2-complex of the dihedral group $\mathcal{D}_{3}$ of order 6.

The spectral dual pair (Copernicus I) is fundamental for the processes of dynamic quantization, kinematic relativization and visuospatial dynamics of artificial intelligence. The central invariant or squared orbital angular momentum operator (Casimir) identity can be conceived as a spectral version of the third order principle of spinor triality to afford a rigorous mathematical treatment of the Foucault's spherical pendulum experiment of dual contact holonomy in terms of the orbital angular momenta via the de Coriolis alternating trilinear form of the ratio orbium to afford the $\varphi_{0}$-latitudinal phase angle displacement $\Delta \theta$ of the of the spin echo-stabilized, symplectic swingplane. The Cayley-Dickson construction suggests to use the spectral dual pair of real Lie groups with mutual commutant actions

$$
(\operatorname{Mp}(2, \mathbb{R}), \operatorname{PSO}(1,9, \mathbb{R}))
$$

to deterimine the full automorphism group of spinor triality. In the context of commutants, the pairing is in the sense of von Neumann $L^{2}$-algebras.

In the field of computational neuroscience, the stage is set for

Theorem 4 The third order principle of spinor triality determines conformally equivariants of navigational information processing of the entorhinal-hippocampal cortex cerebri at long terms and large ranges within the octonion projective manifold $\mathbb{P}_{\mathbb{O}}\left(\mathfrak{L i} \mathfrak{i}(\mathcal{N})^{*}\right)$ of visuospatial states under readout by visuospatial memory performance of the hippocampal microcircuitry.

The profoundness of the spectral duality (Copernicus I) goes into the simultaneous development of symplectic contact geometry via the spin structure on the tangent bundle $\mathrm{TS}_{2}$ of the supporting oriented Riemann contact sphere $\mathbb{S}_{2}^{\frac{1}{2}} \hookrightarrow \mathbb{P}_{\mathbb{C}}\left(\mathfrak{L i e}(\mathcal{N})^{*}\right)$ of radius $\frac{1}{2}$, and the orthogonal geometry of temporal-spatial relativistic signature $(1,3)$ and Witt index 4 which intervenes the hidden symmetries defined by the third order principle of spinor triality superseding the symmetries of the spectral duality (Copernicus I). It should be observed that trialities are much rarer and by far subtler than linear dualities and pairings of real projective quadrics because they come from the well categorized spectrum of non-associative alternative normed division algebras over the real number field $\mathbb{R}$. To no surprise, they are playing a role in the supersymmetry investigations of nuclear physics [6].

In clinical nuclear spin magnetic resonance tomography, insertion of the diffusion tensor into the action of the Lorentz-Möbius Lie group $\operatorname{PSO}(1,3, \mathbb{R})$ can be conceived as an extension strategy going analogously from special to general relativity theory. Due to the inclusion of kinematic relativization

$$
\operatorname{PSO}(1,3, \mathbb{R}) \hookleftarrow \operatorname{PSO}(1,1, \mathbb{R}),
$$

a unique combination of hardware, pulsed gradient spin echo trains, magnetic field monitoring and image reconstruction modalities opens up in terms of diffusion ten- 
sor nuclear spin magnetic resonance tomography a new route into microstructural properties in the living human brain, such as the myelin sheats of nerve axons [21,32].

Remark 3 The impact of the third order principle of spinor triality via the theorem of Alexandrov-Ovchinnikova on the dynamics of Poncelet's porism of algebraic geometry infers an explanation of Schrödinger's quantum mechanical cat problem via the closure configuration inside the affine plane $\mathbb{R} \oplus \mathbb{R}$.

The universal covering group $\widetilde{\operatorname{Mp}}(2, \mathbb{R})$ of the Lie group $\operatorname{Sp}(2, \mathbb{R}) \cong \operatorname{SL}(2, \mathbb{R})$ admits the exact sequence

$$
\{1\} \longrightarrow \mathrm{SO}(2, \mathbb{R}) . \mathrm{id} \hookrightarrow \widetilde{\mathrm{Mp}}(2, \mathbb{R}) \longrightarrow \mathrm{Sp}(2, \mathbb{R}) \longrightarrow\{1\}
$$

where $\mathrm{SO}(2, \mathbb{R}) \cong \mathrm{U}(1, \mathbb{C}) \cong \mathbb{S}_{1}$ implements the phase factor of the diurnal rotation of the compact spin manifold $\mathbb{S}_{2}$, and $\operatorname{Mp}(2, \mathbb{R})$ is a closed subgroup of the connected Lie group $\widetilde{\mathrm{Mp}}(2, \mathbb{R})$.

Octonion backstage inspection. The metaplectic group $\operatorname{Mp}(2, \mathbb{R})$ is a connected Lie group which forms a non-trivial two-fold covering group of the symplectic group $\operatorname{Sp}(2, \mathbb{R}) \cong \operatorname{SL}(2, \mathbb{R}) \cong \operatorname{SU}(1,1, \mathbb{C})$ in accordance with the exact sequence

$$
\{1\} \longrightarrow \mathbb{Z}_{2} \cdot \mathrm{id} \hookrightarrow \operatorname{Mp}(2, \mathbb{R}) \stackrel{\mu}{\longrightarrow} \operatorname{Sp}(2, \mathbb{R}) \longrightarrow\{1\}
$$

It sets the backstage for the Cayley-Dickson duplication strategy of improving the resolution of encoded information, and the third order principle of triality of the algebraic theory of spinors and associative Clifford/Graßmann algebra models of orbital angular momenta or torques [10]. The structure identification of the strategy relevant associative Clifford algebra and associated Graßmann algebra models produces a skew-derivation $\delta_{\bullet}$, homogeneous of degree -1 , with the properties

$$
\delta_{\bullet}^{2}=0, \quad \delta_{\bullet} \circ \delta_{\bullet}^{\prime \prime}+\delta_{\bullet}^{\prime \prime} \circ \delta_{\bullet^{\prime}}=0
$$

well-known from de Rham cohomology groups of Borel-Weil theory, and the theory of supersymmetry [6]. The functorial property is in correspondence to the local or infinitesimal version of the third order principle of spinor triality, and its calculus actually forms the cornerstone of the symplectic spinor methodology of ordo orbium via the third Kepplerian spinor law $\mathrm{H}^{3}$ of planetary dynamics. The importance of the contact pairing

$$
\left(\delta_{\bullet}, \bullet \wedge\right)
$$

which consists of the uniquely determined skew-derivation and the symplectic form so that the identity

$$
\bullet \wedge \bullet^{\prime}=-\frac{1}{4}[\bullet, \bullet ']
$$


holds, derives from the fact that it exhibits a linear representation $\rho$ of $\operatorname{Spin}(4, \mathbb{R}) \cong$ $\mathbb{S}_{3} \times \mathbb{S}_{3} \cong \operatorname{Spin}(3, \mathbb{R}) \times \operatorname{Spin}(3, \mathbb{R}) \cong \operatorname{SU}(2, \mathbb{C}) \times \operatorname{SU}(2, \mathbb{C})$ via the associative Clifford algebra of the quadratic form of temporal-spatial relativistic signature $(1,3)$ and Witt index 4. This Clifford algebra reveals to be a central simple algebra. If $\mathbb{O}$ denotes the non-associative alternative normed division algebra of octonions of dimension

$$
\operatorname{dim}_{\mathbb{R}} \mathbb{O}=\operatorname{dim}_{\mathbb{R}} \mathbb{H} \oplus \mathbb{H}=2^{3}=8,
$$

and

$$
\mathfrak{S}=\mathfrak{S}_{-} \oplus \mathfrak{S}_{+}
$$

denotes the real vector space of spinors under its chiral decomposition for the Witt index 4 , then $\mathfrak{S}$ has dimension

$$
\operatorname{dim}_{\mathbb{R}} \mathfrak{S}=2 \operatorname{dim}_{\mathbb{R}} \mathbb{O}=\left(\# \mathfrak{V}_{4}\right)^{2}=2^{4}=16
$$

In this context $\mathfrak{V}_{4}$ presents the non-cyclic Klein four-group which is associated by the ratio orbium of the third order principle of spinor triality to the hexagonal tessellation.

The real vector space

$$
\mathbb{P}_{\mathbb{O}}\left(\mathfrak{L} \mathfrak{i} \mathfrak{e}(\mathcal{N})^{*}\right) \cong \mathbb{P}_{\mathbb{R}}\left(\left(\mathbb{R}^{2} \oplus \mathbb{R}\right) \oplus \mathbb{O} \oplus(\mathbb{O} \oplus \mathbb{O})\right)
$$

admits by the half-spinor space splitting the foliation

$$
\mathbb{P}_{\mathbb{O}}\left(\mathrm{T}_{\bullet}\left(\mathbb{S}_{2}\right)\right) \hookrightarrow \mathbb{P}_{\mathbb{O}}\left(\mathfrak{L i e}(\mathcal{N})^{*}\right)
$$

The real vector space of spinors $\mathfrak{S}$ for the Witt index 4 is decomposed into the direct sum of the non-isotropic vector subspaces of odd pure half-spinors $\mathfrak{S}_{-}$and even pure half-spinors $\mathfrak{S}_{+}$, which have the same dimension 8 as the non-associative alternative normed division algebra of octonions $\mathbb{O} \hookrightarrow \mathbb{P}_{\mathbb{O}}\left(\mathfrak{L i e}(\mathcal{N})^{*}\right)$. The faithful spin representation $\rho$ on $\mathfrak{S}$ gives rise to the construction of a real vector space automorphism of the octonion extension

$$
\mathfrak{L i e}(\mathcal{N})^{*} \otimes_{\mathbb{R}} \mathbb{O}
$$

or equivalently of an $\mathbb{R}$-linear projectivity $j$ of order 3 of the real vector space $\mathbb{P}_{\mathbb{O}}\left(\mathfrak{L} \mathfrak{i}(\mathcal{N})^{*}\right)$, which in conjunction with the underlying quadratic form $\omega$ on the extended vector space

$$
\mathbb{P}_{\mathbb{O}}\left(\mathfrak{L} \mathfrak{i} \mathfrak{e}(\mathcal{N})^{*}\right) \hookleftarrow \mathbb{P}_{\mathbb{C}}\left(\mathfrak{L} \mathfrak{i} \mathfrak{e}(\mathcal{N})^{*}\right)
$$

implements by an extension of the post-Kepplerian theorem of AlexandrovOvchinnikova the global symmetry principle of spinor triality at the highest possible level $3 \cdot 2^{3}$ over its octonionic platform [10]. 
The tensor product $\mathfrak{S} \otimes_{\mathbb{R}} \mathfrak{S}$ of the split real vector space of spinors for the Witt index 4 by itself can be identified with the associated associative Clifford/Graßmann algebra model of real dimension $2^{8}=256$. It gives rise to the central norm homomorphism $\lambda$ via the key notion of bilinear invariant $\beta\left(\bullet, \bullet^{\prime}\right)$ of the spin representation $\rho$ on the product space $\mathfrak{S} \times \mathfrak{S}$, where the equations

$$
\beta(\rho . \bullet, \bullet ')=\lambda . \beta(\bullet, \bullet ')
$$

and

$$
\beta\left|\left(\mathfrak{S}_{-} \times \mathfrak{S}_{+}\right)=\beta\right|\left(\mathfrak{S}_{+} \times \mathfrak{S}_{-}\right)=0
$$

hold. In this framework, the spin representation $\rho$ transforms the real vector subspaces of pure half-spinors into the real vector subspace of octonions $\mathbb{O} \hookrightarrow \mathbb{P}_{\mathbb{O}}\left(\mathfrak{L i e}(\mathcal{N})^{*}\right)$. The half-spinor representations are irreducible and inequivalent.

In conjunction with the simple Lorentz-Möbius Lie group $\operatorname{PSO}(1,3, \mathbb{R}) \hookrightarrow$ $\operatorname{PGL}(1,3, \mathbb{R})$, which includes the temporal aspects of projective optics, the Lie group $\widetilde{\mathrm{Mp}}(2, \mathbb{R})$ implements the angular gauge function of relativistic gravitation in the framework of dynamic quantization [25] under the inherent longitudinal, or axial action of the Witt spinor norm group

$$
\left.\mathrm{WS}(\mathbb{R}) \cong \operatorname{SO}(4, \mathbb{R}) / \operatorname{PSO}(1,3, \mathbb{R}) \cong \mathbb{R}^{\times} / \mathbb{R}^{\times 2} \hookrightarrow \mathrm{H}^{1}(\mathbb{R}, \operatorname{Spin}(3, \mathbb{R}))\right)
$$

of order 2 over the central projective collineations $\mathbb{R} \cong \operatorname{PSO}(1,1, \mathbb{R})$. Thus the action of WS $(\mathbb{R})$ is compatible with the third order principle of spinor triality outlined in terms of the largest of the four normed division algebras, the non-associative alternative division algebra of octonions $\mathbb{O}$, and the associated free Abelian Witt group

$$
\mathrm{WQ}(\mathbb{R}) \cong \mathbb{Z}
$$

permits the application of the Leibniz principle of projective analysis to iteratively create a hierarchy of fields of quantum states, the ordo orbium, within the complex projective manifold $\mathbb{P}_{\mathbb{C}}\left(\mathfrak{L i} \mathfrak{e}(\mathcal{N})^{*}\right)$ of quantum field theory.

In the context of the dual contact holonomy of Foucault's spherical pendulum experiment, the Witt spinor norm group WS $(\mathbb{R})$ and the proof of Hopf's Umlaufsatz for the rotation index $\{ \pm 1\} \cong \mathbb{Z}_{2}$ of a simple plane loop [61] give rise to the concept of real-valued angular gauge function $t \rightsquigarrow \theta(t)$, which is unique up to an additive constant and defines a horizontal parametrization of the parallel transport dynamics of the spin echo-stabilized, symplectic swing-planes caused by the spinning earth and the reduced covariance of the oscillating spherical pendulum device.

The Witt spinor norm group WS $(\mathbb{R})$ over the field of central projective collineations $\operatorname{PSO}(1,1, \mathbb{R}) \cong \mathbb{R}$ is able to clear within the category of free Abelian groups or free $\mathbb{Z}$-modules with basis $\{1\}$ the severe lack of gravitational foundation from which the incipient Copernican heliocentric cosmology suffered. What it actually lacked was a quantum field theoretic approach based on the dual pair (Copernicus I) supra, 
and (Copernicus II) as will be noted infra. In terms of the category of free Abelian groups, quantization associates with the Witt spinor norm group WS $(\mathbb{R})$ the Cartesian product group $\mathbb{Z} \times \mathbb{Z}$ under coordinatewise addition with respect to the canonical basis $\{(1,0),(0,1)\}$ of rank two.

Interpretations of the Copernican revolution have been hampered for a long time by an unexamined refusing of the orbit methodology of contact holonomy. The purpose of the present paper is to elucidate the spherically calibration of the orthogonal contact geometric quantization procedure of rotation-sensing technologies which are intrinsic to the Hopf principal circle bundle [42]

$$
\mathbb{S}_{1} \hookrightarrow \mathbb{S}_{3} \stackrel{\eta_{2}}{\longrightarrow} \mathbb{S}_{2}
$$

implementing the Hopf submersion $\eta_{2}$ over the principal circle bundle

$$
\mathrm{SO}(3, \mathbb{R}) \longrightarrow \mathrm{SO}(3, \mathbb{R}) / \mathrm{SO}(2, \mathbb{R})
$$

over the two-dimensional oriented round sphere $\mathbb{S}_{2} \cong \operatorname{SO}(3, \mathbb{R}) / \operatorname{SO}(2, \mathbb{R}) \cong \mathbb{P}_{1}(\mathbb{C})$ under its spin manifold structure. In terms of the skew-field of ordinary or classical quaternions $q \in \mathbb{H}$, the Hopf submersion admits the form

$$
\eta_{4}: \mathbb{H} \hookleftarrow \mathbb{S}_{3} \ni q \rightsquigarrow q i \bar{q} \in \mathbb{S}_{2} \hookrightarrow \Im(\mathbb{H})
$$

In view of the fact that $\Im(\mathbb{H})$ represents a three-dimensional Euclidean vector space, it follows for the projective group $\operatorname{PSO}(4, \mathbb{R})$ the direct product splitting into simple groups

$$
\operatorname{PSO}(4, \mathbb{R}) \cong \operatorname{SO}(3, \mathbb{R}) \times \operatorname{SO}(3, \mathbb{R}) \cong \operatorname{Aut}(\mathbb{H}) \times \operatorname{Aut}(\mathbb{H})
$$

In other words, the Lie groups $\mathrm{SO}(4, \mathbb{R})$ and $\mathbb{S}_{3} \times \mathrm{SO}(3, \mathbb{R})$ are non-isomorphic twofold covering groups of the direct product group $\mathrm{SO}(3, \mathbb{R}) \times \mathrm{SO}(3, \mathbb{R})$.

To put the spherically calibrated contact geometry in the less concise language than the Copernican cosmology could have reestablished from the Pseudo-Aristotelean treatise Qucestiones mechanica, it is the law of ratio orbium which discharges later on into the contact geometry of the third Kepplerian spinor law $\mathrm{H}^{3}$ of planetary dynamics.

The phase framing gyroscopic contact torus of revolution $\mathbb{S}_{1} \times \mathbb{S}_{1}$ consists of the $\eta_{2}$-preimage of a reference circle on the two-dimensional compact spin manifold or canonical complex line bundle

$$
\mathbb{S}_{2} \cong \mathrm{O}(3, \mathbb{R}) / \mathrm{O}(2, \mathbb{R}) \cong \mathrm{SO}(3, \mathbb{R}) / \mathrm{SO}(2, \mathbb{R}) \cong \mathbb{P}_{1}(\mathbb{C})
$$

at latitude direction angle $\varphi \in] 0, \pi$ [ in the very round sphere model

$$
\mathbb{S}_{3} \cong \mathrm{O}(4, \mathbb{R}) / \mathrm{O}(3, \mathbb{R}) \cong \operatorname{SO}(4, \mathbb{R}) / \mathrm{SO}(3, \mathbb{R}) \cong \operatorname{Spin}(3, \mathbb{R}) \cong \mathrm{SU}(2, \mathbb{C})
$$

of rotation-sensing technologies. The Clifford immersion of the flat torus $\mathbb{S}_{1} \times \mathbb{S}_{1}$ into the very round sphere model $\mathbb{S}_{3} \hookrightarrow \mathbb{R}^{4} \cong \mathbb{C}^{2}$ gives rise to the supplementary open- 
book foliation in the sense of contact topology with covariant pages and vertically operating free Abelian Witt group WQ(R) over the real number field field of central projective collineations $\operatorname{PSO}(1,1, \mathbb{R}) \cong \mathbb{R}$. It realizes projectively the inscription

Terræ Motor Solis Cælique Stator

which stands on the base of the Copernicus monument in Thorn since 1853 and corresponds literally to the formula of abjunction that was imposed upon Galilei by the Roman censorship in 1633 to joit the solar system. Due to the principle of circular inertia, the basic idea of Foucault's spherical pendulum holonomy is the dynamics of its spin echo-stabilized, symplectic swing-planes horizontally parametrized by the parallel transport of the covariant pages of the open-book foliation with $\mathbb{S}_{1} \cong \mathbb{T}$-binding, which actually forms a geometric interpretation of the unitarily induced representation mechanism in terms of the theory of Schwartz kernels.

With respect of the duality aspect of the inscription, it is important to observe that Copernicus did not arrive at the standpoint of the relativity of motion. The postCopernican standpoint of kinematic relativization has been achieved by Leibniz in his highly sophisticated treatise entitled Tentamen de motuum colestium causis of 1689. In view of the fact that the Lorentz-Möbius Lie group $\operatorname{PSO}(1,3, \mathbb{R})$ implements the temporal-spatial aspect of kinematic relativization, the unexpected impact of the Tentamen essay on the causes of celestial motions is the introduction of the concept of temporal-spatial relativistic signature of symmetric bilinear forms. This cunning Promemoria implicitly gives rise via Hermann Minkowski's indefinite quadratic form approach to kinematic relativization to the notion of Witt spinor norm group WS $(\mathbb{R}) \cong$ $\mathbb{R}^{\times} / \mathbb{R}^{\times 2}$ and its inherent projective stellarization action along the gyroscopic axis of the spinor gauge of relativistic gravitation. The signature isomorphism of the free Abelian Witt group WQ( $\mathbb{R})$ onto the additive group $\mathbb{Z}$ of rational integers permits to establish the dynamic spin echo-stability of the Foucault spherical pendulum device. The modern theory of quadratic forms, which has been opened by Ernst Witt (19111991) admits a new application in the quantization of the Foucault spherical pendulum.

\section{Copernican spherical holonomy and Kepplerian calibrated contact geometry}

Copernicus wrote in the dedicatory foreword of his opus magnum of 1543 De revolutionibus orbium colestium. Prafatio ad Paulum III: "When I had pondered for a long time this uncertainty of the traditional mathematics in connection with the calculation of the motions of the round spheres, it came to seem scandalous that in spite of such painstaking investigation of the most trivial data regarding that circular motion, the philosophers had not arrived at a more dependable idea of the movement of the world's machinery, even though this was constructed on our behalf by the best and most trustworthy Master Builder of everything".

Although the work of the neo-Pythagorean Copernicus was predominantly scientific, its phenomenological consequences, of course, extended far beyond science generating universal metaphors like that of man's "removal from the center of the cos- 
mos". However, Copernicus is not, for Immanuel Kant (1724-1804) at least, one of those real transformers of scientific reasoning, because he changed the world-model, not the methodology for setting up and handling models. Of course, the skándalon referred to by Copernicus occurred much earlier than Galilei's contact topological paradigm of supplementary open-book foliation started to be known as a temporal storage medium "so that the reading of the gorgeous concepts that are written in the pages of the foliation would not be completed by merely seeing the splendor of the sun and the stars and their rising and setting, but also by the studies of hundreds of hundreds of the most acute minds" inside the covariant pages, and by the discovery of the very round sphere in the space $\mathbb{R}^{4} \cong \mathbb{C}^{2}$, which revealed to be an active ingredient of the mathematical treatment of the Copernican cosmology. Nevertheless, the Copernican novel claim to cosmic truth was of incomparable importance for Keppler's subsequent development of the incipient mathematical astrophysics and its cosmological models.

In the context of the inscription of the Copernicus monument in Thorn since 1853, the two-dimensional complexified sphere $\mathbb{S}_{2}^{\mathbb{C}} \cong \operatorname{SO}(3, \mathbb{C}) / \mathrm{SO}(2, \mathbb{C})$ gives rise to the central driving invariant or squared orbital angular momentum operator (Casimir) by means of the Poincaré-Birkhoff-Witt theorem, which identifies the universal enveloping graded Lie algebra of the symplectic group $\operatorname{Sp}(2, \mathbb{C}) \cong$ $\operatorname{SL}(2, \mathbb{C})$ with the symmetric algebra $\operatorname{Sym}_{\mathbb{C}}(\mathfrak{L} \mathfrak{i} \mathfrak{e}(\operatorname{Sp}(2, \mathbb{C})))$ of $\mathfrak{L} \mathfrak{i} \mathfrak{e}(\operatorname{Sp}(2, \mathbb{C}))$ over the field $\mathbb{C}$ of complex numbers. The adjoint representation reveals to be the group epimorphism $\mathrm{SL}(2, \mathbb{C}) \longrightarrow \mathrm{SO}(3, \mathbb{C})$ corresponding to the Lie algebra homomorphism $\mathfrak{L} \mathfrak{i} e(\operatorname{SL}(2, \mathbb{C})) \longrightarrow \mathfrak{L i e}(\operatorname{SO}(3, \mathbb{C}))$. In this way, to wit the transitive action of the special orthogonal Lie group $\mathrm{SO}(3, \mathbb{C})$ on the complexified circle bundle $\mathrm{SO}(3, \mathbb{C}) \longrightarrow \mathrm{SO}(3, \mathbb{C}) / \mathrm{SO}(2, \mathbb{C})$, the Kepplerian planetary contact holonomy remediates the Copernican cosmology of the lack of gravitational foundation that Kant has criticized.

Keppler was to be the first to comprehend the fact that the logic of his radical Copernicanism entailed not only reversing the relation of appearance and reality in the relation of the starry heavens to the earth but also adapting the energy relationship to this inversive geometry. He recognized this reversal when he made the dynamics of the spinning system emanate from the center of the universe, to wit, when he made it proceed from the inside outward. Thus Keppler's claim to cosmic truth is based on a higher level of explanatory accomplishment and reveals the task that Newton later on will set for himself in order to confirm the Copernican reform by means of the causal relationships within it. It is the Hopf fibration over the two-dimensional homogeneous manifold $\mathbb{S}_{2} \cong \operatorname{SO}(3, \mathbb{R}) / \mathrm{SO}(2, \mathbb{R})$, and the supplementary open-book foliation, affiliated with the complex numbers, which allow to unify conformally the various aspects of the Copernican cosmology.

The spinor approach to the Kepplerian claim to cosmic truth has been accomplished at the higher level of explanatory accomplishment by the third order principle of spinor triality. It reveals that even the task that Newton set for himself in order to confirm the Copernican heliocentric system by means of the causal relationships within it deserves deeper spinorial insight. Thus the magnificent definition of spinor due to Élie Cartan (1865-1951), who changed the model and the method in 1925, and the key concept of control by the Witt norm have their cosmic origin in De revolutionibus orbium colestium almost four centuries earlier. The lack of a mathematically rigorous concept 
of a real vector space $\mathfrak{S}$ of spinors, which permits the creation of a suitable symmetry principle of spinor triality, prevented the chief work De revolutionibus... to establish the role of a standard reference of mathematical astrophysics alike Newton's Philosophice Naturalis Principia Mathematica of 1687, which presents his fundamental law of universal gravitation. The associated quadratic form is given by the square of the distance between the masses.

In a comparison that is hardly surpassable in terms of the scientific influence it has exercised, Kant's analysis of the Copernican cosmology emphasized that it is only in connection with the annual motion that is makes sense to speak of the sun's revolving around the spinning earth being replaced by the spinning earth's revolving around the sun. It is true that the spinning earth's axial eigen-motion takes responsibility for the sun's diurnal motion across the sky, but it does not allow to state that the reverse is the case: the spinning earth revolves around the sun. Thus the initial Copernican reform is concerned with the consistently narrow thesis of the spinning earth's axial eigen-motion of the diurnal rotation.

Under the Newtonian perspective of the Copernican cosmology, there could not exist a free arrangement of forces, because for Newton the configuration of the bodies in the planetary system was an expression of the highest wisdom so that the ordo orbium therefore could not be the outcome of a stochastic process. On the contrary, it was a sophisticated calculus that intended, by means of a gravitational fall from a certain distance, an orthogonal deflection to one side and a duplication of the gravitational force of the central body to the other side, to cause the planet-to-be to swivel into the orbit provided for it, that is, into the orbit established in accordance with the third Kepplerian spinor law $\mathrm{H}^{3}$ of planetary dynamics with its roots in the non-trivial twofold covering group $\operatorname{Mp}(2, \mathbb{R})$ of the symplectic group $\operatorname{Sp}(2, \mathbb{R}) \cong \operatorname{SL}(2, \mathbb{R})$, and the free Abelian Witt group WQ $(\mathbb{R}) \hookrightarrow \operatorname{PSO}(1,3, \mathbb{R})$ over the field of central projective collineations $\operatorname{PSO}(1,1, \mathbb{R}) \cong \mathbb{R}$.

\section{Prequantization: the metaplectic coadjoint orbit model of the unitary dual $\widehat{\mathcal{N}}$}

Denote by $\mathcal{N}$ the $(2+1)$-dimensional connected and simply connected Lie group consisting of the upper triangular unipotent matrices

$$
\left\{\left(\begin{array}{llc}
1 & w & \frac{v}{2 i} \\
0 & 1 & \bar{w} \\
0 & 0 & 1
\end{array}\right)=\left.\exp \left(\begin{array}{llc}
0 & w & \frac{z}{2 i} \\
0 & 0 & \bar{w} \\
0 & 0 & 0
\end{array}\right)|w \in \mathbb{C}, v=z+i| w\right|^{2}, z \in \mathbb{R}\right\}
$$

with one-dimensional center $\mathcal{Z} \hookrightarrow \mathcal{N}$, and real Lie algebra $\mathfrak{L} \mathfrak{i}(\mathcal{N})$ with center $\mathfrak{L i} \mathfrak{e}(\mathcal{Z}) \hookrightarrow \mathfrak{L} \mathfrak{i}(\mathcal{N})$. As a group extension, $\mathcal{N} \hookrightarrow \operatorname{GL}(3, \mathbb{R})$ appears in the form of the injective homomorphism

$$
\mathbb{R} \rtimes \mathcal{N} \longrightarrow \mathbb{R} \oplus \mathbb{R} \cong \mathbb{C}
$$


or the short exact sequence

$$
\{0\} \longrightarrow \mathbb{R} \longrightarrow \mathcal{N} \longrightarrow \mathbb{C} \longrightarrow\{0\}
$$

Together with a 2-cocycle $\mathbb{C} \times \mathbb{C} \longrightarrow \mathbb{R}$ it gives rise to the second Galois cohomology $\mathrm{H}^{2}(\mathbb{C}, \mathbb{R})$ of the central group extension $\mathbb{C} \mid \mathbb{R}$. The normal subgroup $\mathbb{R}$ is represented the set of matrices

$$
\mathfrak{L} \mathfrak{i} e(\mathcal{Z})=\left\{\left(\begin{array}{lll}
0 & 0 & z \\
0 & 0 & 0 \\
0 & 0 & 0
\end{array}\right) \mid z \in \mathbb{R}\right\} \cong \mathbb{R},
$$

so that the exponential mapping

$$
\exp : \mathfrak{L i e}(\mathcal{N}) \longrightarrow \mathcal{N}
$$

is a diffeomorphism which respects the group law of $\mathcal{N}$. In terms of the skew bilinear Lie bracket or commutation bracket of $\mathfrak{L} \mathfrak{i}(\mathcal{N})$, the homogeneous triality condition

$$
[[\mathfrak{L} \mathfrak{i} \mathfrak{e}(\mathcal{N}), \mathfrak{L} \mathfrak{i} \mathfrak{e}(\mathcal{N})], \mathfrak{L} \mathfrak{i} \mathfrak{e}(\mathcal{N})]=0,
$$

and the basic Heisenberg uncertainty principle read

$$
[\mathfrak{L} \mathfrak{i} \mathfrak{e}(\mathcal{N}) / \mathfrak{L} \mathfrak{i} e(\mathcal{Z}), \mathfrak{L} \mathfrak{i} e(\mathcal{N}) / \mathfrak{L} \mathfrak{i} e(\mathcal{Z})]=\mathfrak{L} \mathfrak{i} e(Z) .
$$

The structure of the Heisenberg unipotent Lie group $\mathcal{N}$ is determined by the semi-direct product of a two-dimensional closed normal Abelian subgroup with a one-dimensional closed subgroup. Their real parametrizations $\left(0, \bullet^{\prime}, \bullet\right)$ and $\left(\bullet^{\prime \prime}, 0,0\right)$, respectively, of the supplementary open book-foliation are determined by the geometric interpretation of the inducing mechanism of unitary linear group representations [25].

Consider the real dual vector space

$$
\mathfrak{L i e}(\mathcal{N})^{*}=\operatorname{Hom}_{\mathbb{R}}(\mathfrak{L} \mathfrak{i} \mathfrak{e}(\mathcal{N}), \mathbb{R})
$$

of the real nilpotent Heisenberg algebra $\mathfrak{L} \mathfrak{i}(\mathcal{N})$ over the real number field field of central projective collineations $\operatorname{PSO}(1,1, \mathbb{R}) \cong \mathbb{R}$ as a hyperplane of the exceptional four-dimensional real vector space $\mathbb{R}^{4} \cong \mathbb{R}^{2} \oplus \mathbb{R}^{2}$, and let $\widehat{\mathcal{N}}$ be the unitary dual of $\mathcal{N}$ consisting of the equivalence classes of its irreducible unitary linear representations. Then the metaplectic Lie group $\operatorname{Mp}(2, \mathbb{R})$ performs a natural horizontal action on the spectrum $\widehat{\mathcal{N}}$ of equivalence classes by the covariance identity of the symplectic group $\operatorname{Sp}(2, \mathbb{R})$ with well defined unitary actions on the complex projective space $\mathrm{PL}_{\mathbb{C}}^{2}(\mathbb{R})$. In the context of $\mathrm{PL}_{\mathbb{C}}^{2}(\mathbb{R})$, it is to be noted that the action of an ordinary unitary linear representation gives rise to a projective representation by just looking at the action on lines. Cooordinatization of the irreducible unitary linear representations of $\mathcal{N}$ reveals an open-book foliation along the center $\mathcal{Z} \hookrightarrow \mathcal{N}$ with $\mathbb{S}_{1} \cong \mathbb{T}$-binding. 
Originally the orthodox interpretation of quantum mechanics dominated with its strict distinction between the classical microscopic world and the microscopic quantum realm. However, the mainstream attitude of the physics community concerning the interface between quantum and classical world changed with the emphasis that actually there is no need for denying quantum physics to hold even macroscopically, and with the understanding of the metaplectic coadjoint orbit model of the unitary dual $\widehat{\mathcal{N}}$ and the supplementary open-book foliation. Information theory of quantum channels has given a new boom to a geometric study of the concept of quantum entanglement in terms of entangled dimetric quantum states [37], and then to engross in a study of the interface between quantum and classical physics. The profoundness of the metaplectic coadjoint orbit model relies on the mapping

$$
\kappa: \mathfrak{L i e}(\mathcal{N})^{*} / \operatorname{CoAd}(\mathcal{N}) \longrightarrow \widehat{\mathcal{N}}
$$

which denotes the natural bijection between the spherical orthogonal contact orbit space

$$
\mathfrak{L i e}(\mathcal{N})^{*} / \operatorname{CoAd}(\mathcal{N})
$$

in the three-dimensional real spherically quadratic vector space dual $\mathfrak{L} \mathfrak{i}(\mathcal{N})^{*}$ of $\mathfrak{L i e}(\mathcal{N})$ under the coadjoint action

$$
\operatorname{CoAd}(\bullet)={ }^{\mathrm{t}} \operatorname{Ad}\left((\bullet)^{-1}\right)
$$

of the real vector space $\mathcal{N}$, and the unitary dual $\widehat{\mathcal{N}}$ of $\mathcal{N}$ acting as the spectrum of $\mathcal{N}$. A generic metaplectic coadjoint orbit $\mathcal{O}_{\bullet} \in \mathfrak{L} \mathfrak{i} e(\mathcal{N})^{*} / \operatorname{CoAd}(\mathcal{N})$ is an algebraic variety in $\mathfrak{L} \mathfrak{i e}(\mathcal{N})^{*}$ homeomorphic either to a single point or to a symplectic plane. The siting of the single point orbits conceived as Dirac point measures collapsing on a homogeneous plane is the singular coadjoint orbit $\mathcal{O}_{\infty} \hookrightarrow \mathfrak{L i e}(\mathcal{N})^{*}$ of projective measurement of spinor based information transport and processing along the attributed non-classical quantum channels.

Embedded into the affine tomographic platform $\mathbb{P}_{\mathbb{C}}\left(\mathfrak{L} \mathfrak{i} \mathfrak{e}(\mathcal{N})^{*}\right)$ of spinor triality, the concept of projective quadrics defines the metaplectic coadjoint orbit model $\mathfrak{L i e}(\mathcal{N})^{*} / \operatorname{CoAd}(\mathcal{N})$ of the unitary dual $\widehat{\mathcal{N}}$ as follows:

Theorem 5 Conceiving the vectors of the three-dimensional real affine space as the central projective collineations of the projective space $\mathbb{P}_{\mathbb{C}}\left(\mathfrak{L i e}(\mathcal{N})^{*}\right)$ having all their fixed points in the plane of infinity, the metaplectic coadjoint orbit model $\mathfrak{L i e}(\mathcal{N})^{*} / \operatorname{CoAd}(\mathcal{N})$ of the unitary dual $\widehat{\mathcal{N}}$ represents modulo $\mathcal{O}_{\infty} \hookrightarrow \mathfrak{L i e}(\mathcal{N})^{*}$ as a real projective quadric of rank two with rank one of the trace on the two-dimensional affine plane at infinity, in conjunction with a real projective quadric with one positive eigenvalue and a real projective quadric with one positive eigenvalue at the affine two-dimensional plane at infinity.

The basic Heisenberg uncertainty principle excludes in terms of the commutation bracket of the $(2+1)$-dimensional real nilpotent Heisenberg Lie algebra $\mathfrak{L i k}(\mathcal{N})$ the 


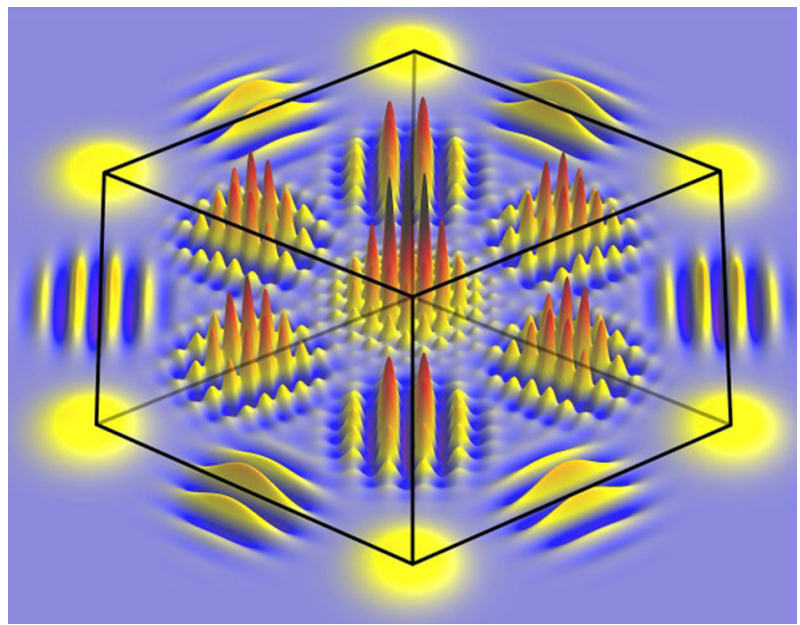

Fig. 4 Lightlike fringe visibility of classical and quantum optical symmetries in terms of central projective collineations of interferometry: Cubic fringe ruling $\mathcal{D}_{3}$ of three photons generated by bosonic $\mathbb{Z}_{2}$-twisted modes that are calibrated by the optomechanical oscillation spectrum of an ultra-thin and small high-stress silicon nitride membrane $\mathrm{Si}_{3} \mathrm{~N}_{4}$ under the interaction of radiation-pressure of cubic shape and the nonlinearity of photon counting. The membrane, embedded in a $10 \times 10 \mathrm{~mm} \mathrm{Si-frame,} \mathrm{is} \mathrm{mounted} \mathrm{in} \mathrm{a} \mathrm{way} \mathrm{that}$ allows optical access from both sides. The quantum interference fringes are generated via two-port photon counting on two Mach-Zehnder type interferometer outputs which project the field inside the interferometer onto a path-entangled photon number state. The high resolution quantum drum over the affine tomographic platform has been sampled by a laser beam to distinguish classical oscillation states and quantum optical states. The photons are reflected either by the device itself or a mirror so that by this artifice only a single photon could traverse simultaneously both optical trajectories to produce the ruled cubic interference fringe pattern. The orbital angular momenta of the photons are in correspondence to a basic triple of octonions in the sense of the third order principle of spinor triality. The mathematical origin of the lightlike dimetric and trimetric poise projection states of Schrödinger's quantum mechanical cat lies in Johannes Keppler's great treatise entitled "Harmonices mundi. Libri V" of 1619. The affine double light-ray cone configuration is in accordance with the fundamental theorem of Alexandrov-Ovchinnikova of relativity theory and its spectral flattening procedure of tomographic affinization by means of the axis $\operatorname{PSO}(1,1, \mathbb{R})$ of central projective collineations to display the central quantum compass state

existence of a quantum state that corresponds precisely to a phase space point. Nevertheless, quantum entanglement highlights the phenomenon of collapse of quantum state in a specially subtle way because it involves pairs of projective measurements on the output of attributed non-classical quantum channels with at least two degrees of freedom or components [34,38,39], (Fig. 4).

Corollary Quantum state collapses occur on the singular homogeneous double plane $\mathcal{O}_{\infty} \hookrightarrow \mathfrak{L i e}(\mathcal{N})^{*}$ as a real projective quadric of rank one with rank one of the trace on the two-dimensional affine plane at infinity, in conjunction with a real projective quadric with one positive eigenvalues and a real projective quadric with one positive eigenvalue at the two-dimensional affine plane at infinity.

Every irreducible unitary linear representation of the Heisenberg unipotent Lie group $\mathcal{N}$ is unitarily equivalent to either a unitarily induced representation or to a unitary character. These representations of $\mathcal{N}$ are evidently pairwise inequivalent. Each 
symplectic plane $\mathcal{O}_{\bullet} \in \mathfrak{L i e}(\mathcal{N})^{*} / \operatorname{CoAd}(\mathcal{N})$ can be viewed as a two-dimensional universal hyperbolic space which admits a contragredient planar copy under the twistor action [5]. The generic metaplectic coadjoint orbit $\mathcal{O} \bullet \hookrightarrow \mathbb{P}_{\mathbb{C}}\left(\mathfrak{L i e}(\mathcal{N})^{*}\right)$ is the operational platform of tomographic filter bank construction and the isotropic symbolic calculus of quantum field theory. It represents the functional analytic counterpart of the arithmetic theory of quadratic forms with its long and tangled history. It is noteworthy that the orthogonal contact geometry in $\mathbb{P}_{\mathbb{C}}\left(\mathfrak{L i e}(\mathcal{N})^{*}\right)$ of Witt's inspiring work on the orthogonal calculus of real quadratic vector spaces $[5,10]$ can be traced back to its roots in mathematical astrophysics. His work is actually able to extend the Copernican reform idea to the emphasis on treating the spinning earth as like another star. The systematic principle of projective stellarization of the spinning earth within the octonion manifold $\mathbb{P}_{\mathbb{O}}\left(\mathfrak{L i e}(\mathcal{N})^{*}\right)$ needs to go deeply into the structure of the projective manifold on which the spinor triality is defined.

Remark 4 The $(2+1)$-dimensional real Heisenberg unipotent and hence exponential solvable Lie group $\mathcal{N}$ does not in any sense correspond to a group of invariances of the physical configuration and its action does not commute with any non-zero Hamiltonian operator; there are no quantum states invariant under the action of $\mathcal{N}$. Quantum information theory, however, provides in terms of nuclear spin magnetic resonance tomography a useful illustration of the metaplectic coadjoint orbit model of the unitary dual $\widehat{\mathcal{N}}$ of equivalence classes of irreducible unitary linear representations of $\mathcal{N}$. According to the great number theorist André Weil (1906-1998), the symplectic group $\mathrm{Sp}(2, \mathbb{R})$ forms the deus ex machina of the theory of quadratic forms due to the investigations of Carl Ludwig Siegel (1896-1981) [69]. The passage from $\widehat{\mathcal{N}}$ with the covariantly acting central extension of the symplectic group $\operatorname{Sp}(2, \mathbb{R})$, which is the metaplectic Lie group $M p(2, \mathbb{R})$, to the central simple associative $\mathbb{R}$-algebras with the Brauer group $\operatorname{Br}(\mathbb{R})$ of distinct isomorphy classes of central simple $\mathbb{R}$-division algebras, however, allows to refer to Galois cohomology which plays a very basic role in class field theory.

As a result, the metaplectic coadjoint orbit model will offer a perspective to the duality of the idele/adele formalism of global-local class field theory to deal with the basic entangling symmetries inherent to the non-invasive modality of nuclear spin magnetic resonance tomography in terms of the third order principle of spinor triality.

\section{The main structure theorem}

The aforementioned geometric transfer summarizes the central aspects of the harmonic analysis on the $(2+1)$-dimensional real Heisenberg unipotent Lie group $\mathcal{N}$ in terms of local class field theory. It prepares the way to the ultrasensitive interferometry of the quantized Foucault spherical pendulum device which illustrates the important concept of cyclic Galois extension of the groundfield $\mathbb{R}$ by factor classes.

Scholium 1. The transfer of the equivalence classes of irreducible unitary linear representations of $\mathcal{N}$ onto the Brauer group $\operatorname{Br}(\mathbb{R})$ of classes of finite dimensional central simple associative algebras over $\mathbb{R}$ and identified with the equivalent Abelian group $\mathrm{H}(\mathbb{R})$ of factor-classes of $\mathbb{R}$ endowed with its Hasse invariants in the twofold Brauer 
groups $\mathrm{Br}_{2}(\mathbb{C}) \hookrightarrow \mathrm{Br}_{2}(\mathbb{R})$ gives rise to the third Galois cohomology of the metaplectic coadjoint orbit model of the unitary dual $\widehat{\mathcal{N}}$ of $\mathcal{N}$ within the dual $\mathfrak{L} \mathfrak{i}(\mathcal{N}) *$ of the $(2+1)$-dimensional real nilpotent Heisenberg algebra $\mathfrak{L} \mathfrak{i}(\mathcal{N})$. Thus the Brauer correspondence links the machinery of Galois group cohomology with nilpotent harmonic analysis.

From the point of view of motion energy it reveals to be of interest that the generic metaplectic coadjoint orbit $\mathcal{O}_{\bullet} \in \mathfrak{L i e}(\mathcal{N})^{*} / \operatorname{CoAd}(\mathcal{N})$ is a symplectic linear variety if, and only if $\kappa\left(\mathcal{O}_{\bullet}\right)$ defines a square integrable representation of $\mathcal{N} / \operatorname{ker}\left(\kappa\left(\mathcal{O}_{\bullet}\right)\right)$. This statement reduces to the strong Stone-von Neumann theorem of the $(2+1)$ dimensional Heisenberg unipotent Lie group $\mathcal{N}$ with associated $(2+1)$-dimensional real nilpotent Heisenberg algebra $\mathfrak{L i e}(\mathcal{N})$ of vanishing Killing form, and the tomographic foliation $\mathfrak{L} \mathfrak{i}(\mathcal{N})^{*} / \operatorname{CoAd}(\mathcal{N})$ longitudinally parametrized along the real line $\mathfrak{L i} \mathfrak{i}(\mathcal{Z}) \hookrightarrow \mathfrak{L} \mathfrak{i}(\mathcal{N})$ [45]. The first integral homology class of the logarithmic symbol associated with $\widehat{\mathcal{N}}$ on the punctured center $\mathcal{Z}-\{0\} \hookrightarrow \mathcal{N}$ points the direction for proceeding further to the deformation of algebras in order to better understand the first Kepplerian spinor law.

Theorem 6 The symplectic form of the generic metaplectic coadjoint orbit $\mathcal{O}_{\bullet} \in$ $\mathfrak{L i e}(\mathcal{N})^{*} / \operatorname{CoAd}(\mathcal{N})$ transforms the symplectic plane $\mathcal{O} \bullet \hookrightarrow \mathbb{P}_{\mathbb{C}}\left(\mathfrak{L i e}(\mathcal{N})^{*}\right)$ into a hyperbolic plane.

The hyperbolic plane is actually the simplest and most important example of an isotropic quadratic space with trivial radical $\{0\}$. The exposition of the mathematical foundations of the Foucault spherical pendulum experiment of dual contact holonomy exploits Hopf-Rinow type minimizing geodesic trajectories which appear as inertial traces designed by spin echo-stabilized, symplectic swing-planes on the terrestrial circular floor panel which is situated beneath the spherical pendulum and where the linear traces are designing diameters of the spin structure induced Poincaré conformal open disc model of the hyperbolic plane.

The Lie group $\mathrm{SU}(1,1, \mathbb{C})$ consisting of the complex matricess

$$
\left\{\left.\left(\begin{array}{cc}
\alpha & \beta \\
\bar{\beta} & \bar{\alpha}
\end{array}\right)|| \alpha\right|^{2}-|\beta|^{2}=1\right\}
$$

is conjugate to $\operatorname{SL}(2, \mathbb{R})$ within $\operatorname{SL}(2, \mathbb{C})$ :

$$
\left(\begin{array}{ll}
1 & i \\
i & 1
\end{array}\right) \operatorname{SU}(1,1, \mathbb{C})\left(\begin{array}{ll}
1 & i \\
i & 1
\end{array}\right)^{-1}=\operatorname{SL}(2, \mathbb{R})
$$

Here $\operatorname{SU}(1,1, \mathbb{C}) \cong \operatorname{SL}(2, \mathbb{R})$ acts on the open unit disc $\{w \in \mathbb{C}|| w \mid<1\}$ via the prescription

$$
w \rightsquigarrow\left(\begin{array}{cc}
\alpha & \beta \\
\bar{\beta} & \bar{\alpha}
\end{array}\right)\left(\begin{array}{l}
w \\
1
\end{array}\right)=\frac{\alpha w+\beta}{\bar{\beta} w+\bar{\alpha}}
$$

leaving the boundary circle $\mathbb{S}_{1} \hookrightarrow \mathbb{C}$ invariant. The action by linear fractional transformations on the unit disc performs in the same way that $\operatorname{SL}(2, \mathbb{R})$ acts on the open 
upper conplex half-plane $\{w \in \mathbb{C} \mid \mathfrak{s} w>0\}$. Under the conjugation, the subgroup of $\mathrm{SU}(1,1, \mathbb{C})$ consisting of the matrices

$$
\left(\begin{array}{cc}
e^{\pi i t} & 0 \\
0 & e^{-\pi i t}
\end{array}\right) \quad(t \in \mathbb{R})
$$

is a maximal compact subgroup which operates on the open unit disc by the rotation $\mathbb{R} \ni t \rightsquigarrow e^{2 \pi i t} w$, where $w \in \mathbb{C}$ and $|w|<1$, and defines the one-dimensional unitary linear representation $\mathbb{C} \ni w \rightsquigarrow e^{2 \pi i t} w \in \mathbb{C}$, where $t \in \mathbb{R}$. On the diagonal are placed complex conjugate unitary characters which give rise to the tempered Schwartz kernels of the Fourier cotransform $\overline{\mathcal{F}}_{\mathbb{R}}$ and Fourier transform $\mathcal{F}_{\mathbb{R}}$ acting as filters on the complex Hilbert space $L^{2}(\mathbb{R})$ of square integrable signals [45]. For pure quantum states the position and momentum probability distributions are Fourier transforms of one another. Thus the bimodal probability dstributions generated by the superposition of identity and parallel displacement operators give rise to the interference fringes in the platform position quadrature.

The minimizing property of geodesics appears with respect to the Poincaré metric of the open disc model. The inverse stereographic projection from the two-sheeted conformal anti-de-Sitter space-time allows to consider the Lorentz-Möbius Lie group

$$
\operatorname{PSO}(1,2, \mathbb{R}) \cong \operatorname{PSL}(2, \mathbb{R}) \cong \operatorname{SL}(2, \mathbb{R}) / \mathbb{Z}_{2} \cong \operatorname{SU}(1,1, \mathbb{C}) / \mathbb{Z}_{2}
$$

as isomorphic to the group of orientation-preserving hyperbolic isometries of the conformal open disc model, where $\mathbb{R} \cong \operatorname{PSO}(1,1, \mathbb{R})$ holds.

It follows for the inertially caused Foucault spherical pendulum experiment of dual contact holonomy:

Corollary The progressively changing, symplectic swing-plane as the spinning earth rotates equals the parallel transport of the initial spin echo-stabilized swing-plane which is passing through diameters of the spin structure induced conformal open disc around the field of tangent planes $\mathrm{T}_{\varphi} \mathbb{S}_{2} \subset \mathrm{TS}_{2}$ at latitude direction angle $\left.\varphi \in\right] 0, \pi[$ of the terrestrial globe $\mathbb{S}_{2} \cong \mathrm{SO}(3, \mathbb{R}) / \mathrm{SO}(2, \mathbb{R}) \cong \mathbb{P}_{1}(\mathbb{C})$.

The group of real algebra automorphisms of $\mathfrak{L} \mathfrak{i}(\mathcal{N})$ which act trivially on the one-dimensional center $\mathfrak{L i} \mathfrak{i}(\mathcal{Z})$ identifies with the $\operatorname{symplectic}$ group $\operatorname{Sp}(2, \mathbb{R})$. As one knows, $\operatorname{Sp}(2, \mathbb{R})$ is isomorphic to the special linear group $\operatorname{SL}(2, \mathbb{R}) \cong \operatorname{SU}(1,1, \mathbb{C})$, and the symplectic complexification $\operatorname{Sp}(2, \mathbb{C})$ is isomorphic to the special linear group $\operatorname{SL}(2, \mathbb{C})$. The linear Lie groups $\operatorname{SL}(2, \mathbb{R})$ and $\operatorname{SL}(2, \mathbb{C})$ are simple groups in the sense that they admit no normal subgroup distinct from the unit element and from the whole group itself. The projective unimodular group of two dimensions PSL $(2, \mathbb{C})$ over the field $\mathbb{C}$ of complex numbers reveals to be isomorphic to the simple Lorentz-Möbius Lie group $\operatorname{PSO}(1,3, \mathbb{R}) \hookrightarrow \operatorname{PGL}(1,3, \mathbb{R})$. In symbols, the complex realization

$$
\operatorname{PSO}(1,3, \mathbb{R}) \cong \operatorname{PSL}(2, \mathbb{C}) \cong \operatorname{SL}(2, \mathbb{C}) / \mathbb{Z}_{2}
$$

of the Lorentz-Möbius Lie group $\operatorname{PSO}(1,3, \mathbb{R})$ in the spectral duality (Copernicus I) holds along the attributed quantum channel. Its temporal-spatial signature $(1,3)$ 
demonstrates that the involvement of the time scale from the view point of relativity infers the transition to the field of complex numbers $\mathbb{C}$. Due to the orientation of the generic metaplectic coadjoint orbits $\mathcal{O}_{\bullet} \in \mathfrak{L i e}(\mathcal{N})^{*} / \operatorname{CoAd}(\mathcal{N})$, it follows

Theorem 7 The contact configuration formed by the metaplectic coadjoint orbit model of the unitary dual $\widehat{\mathcal{N}}$ of the $(2+1)$-dimensional Heisenberg unipotent Lie group $\mathcal{N}$ admits the Witt index 4 in the real quadratic vector space dual $\mathfrak{L} \mathfrak{i}(\mathcal{N})^{*}$.

It is known that the 2-cocycle of the metaplectic Lie group $\operatorname{Mp}(2, \mathbb{R})$ over the symplectic group $\operatorname{Sp}(2, \mathbb{R}) \cong \operatorname{SL}(2, \mathbb{R})$ can be calculated ted in terms of the half-spinor Maslov index as differential $\mu_{*}$ of the finite covering epimorphism $\mu: \operatorname{Mp}(2, \mathbb{R}) \longrightarrow$ $\operatorname{Sp}(2, \mathbb{R})$ evaluated at the Fourier transform $\mathcal{F}_{\mathbb{R}}$ [25]. In agreement with the notion of half-spinor Maslov index, the Hopf principal circle bundle $\mathbb{S}_{1} \hookrightarrow \mathbb{S}_{3} \stackrel{\eta_{2}}{\longrightarrow} \mathbb{S}_{2}$ together with the bundle homomorphism

$$
\mathbb{S}_{3} \stackrel{\mathrm{Ad}}{\longrightarrow} \mathrm{SO}(3, \mathbb{R})
$$

gives rise to the following statement:

Corollary The spin structure on the symplectic tangent bundle $\mathrm{TS}_{2}$ implements the Witt index 2 in the complex projective manifold $\mathbb{P}_{\mathbb{C}}\left(\mathfrak{L i e}(\mathcal{N})^{*}\right)$.

In fact, the circular assignment $\rho: \mathbb{S}_{1} \ni e^{i t} \rightsquigarrow e^{2 i t} \in \mathbb{S}_{1}$ defines at the plane bundle level for the time scale $t \in \mathbb{R}$ a two-fold covering of the planar rotation group $\operatorname{SO}(2, \mathbb{R})$ by the spin group $\operatorname{Spin}(2, \mathbb{R})$, which is not universal.

Contiguously to the generic metaplectic coadjoint orbit $\mathcal{O}_{\bullet} \in \mathfrak{L} \mathfrak{i} \mathfrak{e}(\mathcal{N})^{*} / \operatorname{CoAd}(\mathcal{N})$, the set of single points of $\mathfrak{L} \mathfrak{i}(\mathcal{N})^{*} / \operatorname{CoAd}(\mathcal{N})$ constitute a two-sided homogeneous plane $\mathcal{O}_{\infty} \hookrightarrow \mathbb{P}_{\mathbb{C}}\left(\mathfrak{L i e}(\mathcal{N})^{*}\right)$ with radical

$$
\operatorname{rad} \mathcal{O}_{\infty}=\{0\}
$$

within the real quadratic vector space dual $\mathfrak{L} \mathfrak{i}(\mathcal{N})^{*}$, where the singletons are in bijective correspondence to zero-dimensional coadjoint orbits of $\mathcal{N}$ in $\mathfrak{L} \mathfrak{i}(\mathcal{N})^{*}$. The symplectic geometry of the hyperbolic plane $\mathcal{O}_{\infty}$ is associated with a different orthogonal geometry, refined by an additional quadratic form, which is defined by the supporting oriented Riemann contact sphere $\mathbb{S}_{2}^{\frac{1}{2}} \hookrightarrow \mathfrak{L} \mathfrak{i}(\mathcal{N})^{*}$ of radius $\frac{1}{2}$ and its equatorial great circle contained in the two-sided homogeneous plane $\mathcal{O}_{\infty}$. Ignorant of quantum field theory, the preeminent reference plane $\mathcal{O}_{\infty}$ was a supposition underlying all of Keppler's astrophysical theories [56]. In diffusion nuclear spin magnetic resonance tomography, $\mathcal{O}_{\infty} \hookrightarrow \mathbb{P}_{\mathbb{C}}\left(\mathfrak{L i} \mathfrak{i}(\mathcal{N})^{*}\right)$ separates spatially the inserted pairs of dephasing and rephasing gradients of the Stejskal-Tanner diffusion spectroscopy filter bank construction.

All hyperbolic planes in the quadratic vector space $\mathfrak{L i e}(\mathcal{N})^{*}$ are isometric and universal and admit the trivial radical $\{0\}$. As expected, the disclosed singletons of $\mathfrak{L} \mathfrak{i}(\mathcal{N})^{*}$ are one-dimensional characters of the $(2+1)$-dimensional real nilpotent Heisenberg Lie algebra $\mathfrak{L} \mathfrak{i}(\mathcal{N})$, exponentially induced by the correspondence $\kappa$ displayed supra, and therefore annihilate the commutation bracket of $\mathfrak{L i} \mathfrak{i}(\mathcal{N}) \hookleftarrow \mathfrak{L i} \mathfrak{e}(\mathcal{Z})$. 
In the context of the one-dimensional characters of $\mathfrak{L} \mathfrak{i}(\mathcal{N})$, Sagnac's effect of two counterpropagating cavity resonance modes in the two-sided hyperbolic plane $\mathcal{O}_{\infty} \hookrightarrow \mathbb{P}_{\mathbb{C}}\left(\mathfrak{L i e}(\mathcal{N})^{*}\right)$ defines the origin of all laser optic gyroscope technologies ([?]) basically used for navigation purposes.

Remark 5 The universal enveloping algebra of the real Heisenberg nilpotent Lie algebra $\mathfrak{L} \mathfrak{i}(\mathcal{N})$ is isomorphic to the symplectic Clifford algebra with trivial center. The Poincaré-Birkhoff-Witt theorem affords an isomorphism to the symmetric algebra $\operatorname{Sym}_{\mathbb{R}}(\mathfrak{L i} \mathfrak{i}(\mathcal{N}))$ over the field of central projective collineations $\operatorname{PSO}(1,1, \mathbb{R}) \cong \mathbb{R}$.

Summing up, the asset of harmonic analysis of the $(2+1)$-dimensional Heisenberg unipotent Lie group $\mathcal{N}$ and kinematic relativization in the complex projective manifold $\mathbb{P}_{\mathbb{C}}\left(\mathfrak{L} \mathfrak{i} \mathfrak{e}(\mathcal{N})^{*}\right)$ by the spectral duality (Copernicus I) is that they provide an approach to the visualization of odd and even pure half-spinors by means of the actions of Witt groups.

Theorem 8 The metaplectic coadjoint orbit model of the unitary dual $\widehat{\mathcal{N}}$ of the $(2+$ 1)-dimensional Heisenberg unipotent Lie group $\mathcal{N}$ affords in conjunction with the Cayley-Dickson duplication strategy the ladder of imbedded projective manifolds

$$
\mathbb{P}_{\mathbb{R}}\left(\mathfrak{L i e}(\mathcal{N})^{*}\right) \hookrightarrow \mathbb{P}_{\mathbb{C}}\left(\mathfrak{L i e}(\mathcal{N})^{*}\right) \hookrightarrow \mathbb{P}_{\mathbb{H}}\left(\mathfrak{L i e}(\mathcal{N})^{*}\right) \hookrightarrow \mathbb{P}_{\mathbb{O}}\left(\mathfrak{L i e}(\mathcal{N})^{*}\right)
$$

which permits a spinor triality construction in dimensions $3 \cdot 2^{m}$, where $m \in\{0,1,2,3\}$, for $\mathbb{R}=\operatorname{Cliff}(0), \mathbb{C}=\operatorname{Cliff}(1), \mathbb{H}=\operatorname{Cliff}(2)$, and the split case $\mathbb{H} \oplus \mathbb{H}=\operatorname{Cliff}(3)$.

At the level $m=3$ of the Cayley-Dickson duplication strategy, the question inevitably arises as to whether one can draw octonion conclusions from the doubling of the gravitation of the central body on the swiveling into the planetary orbit established in accordance with the third Kepplerian spinor law $\mathrm{H}^{3}$ of planetary motion.

Theorem 9 In the inclinatio contact geometry, the supporting two-dimensional oriented Riemann contact sphere $\mathbb{S}_{2}^{\frac{1}{2}} \hookrightarrow \mathbb{P}_{\mathbb{C}}\left(\mathfrak{L i} \mathfrak{i}(\mathcal{N})^{*}\right)$ of radius $\frac{1}{2}$ gives rise to the non-degenerate quadratic form $\omega$ which underlies the third order principle of spinor triality on the extended vector space $\mathbb{P}_{\mathbb{O}}\left(\mathfrak{L} \mathfrak{i}(\mathcal{N})^{*}\right)$. The quadratic form $\omega$ is invariant under the action of the a real vector space triality automorphism $\mathrm{j}$ of order 3 of $\mathbb{P}_{\mathbb{O}}\left(\mathfrak{L} \mathfrak{i} \mathfrak{e}(\mathcal{N})^{*}\right)$ with $\operatorname{dim}_{\mathbb{R}} \mathbb{P}_{\mathbb{O}}\left(\mathfrak{L} \mathfrak{i} \mathfrak{e}(\mathcal{N})^{*}\right)=24-1$.

Remark 6 The eight-dimensional non-associative alternative normed division algebra of octonion $\mathbb{O}$ is non-associative and therefore does not give rise to the structure of a Clifford algebra. The vector product

$$
\bullet \bar{\wedge} \bullet^{\prime}=\frac{1}{2}\left[\bullet, \bullet^{\prime}\right]
$$

turns $\Im(\mathbb{H})$ into a real Lie algebra, but not $\Im(\mathbb{O})$.

In the context of diagnostic visualization, a striking application of spinor theory is the mathematical foundation of the discipline of clinical nuclear spin magnetic resonance 
tomography [44]. There, and in the whole field of nuclear spin magnetic resonance technology, the spin echo-stabilizing notion of spin echo finds its appropriate symplectic explanation.

\section{The unique structure of the exceptional Jordan algebra}

Notice that the non-associative alternative normed division algebra $\mathbb{O}$ is equipped with a trilinear map, called the associator, which measures the failure of associativity within the division algebra $\mathbb{O}[6]$. Due to the Stone-von Neumann theorem, the octonion projective manifold $\mathbb{P}_{\mathbb{O}}\left(\mathfrak{L} \mathfrak{i} \mathfrak{e}(\mathcal{N})^{*}\right)$ gives rise to the exceptional Jordan algebra $\mathfrak{h}_{3}(\mathbb{O})$ under the pervasive spectral influence of spinor triality:

$$
\widehat{\mathcal{N}} \hookrightarrow \mathbb{P}_{\mathbb{O}}\left(\mathfrak{L i e}(\mathcal{N})^{*}\right) \cong \mathfrak{h}_{3}(\mathbb{O})
$$

In terms of Veronese coordinates [42], the exceptional Jordan algebra $\mathfrak{h}_{3}(\mathbb{O})$ over $\mathbb{R}$ consists of the Hermitian block matrices

$$
A=\left(\begin{array}{ccc}
a & \mathfrak{z} & \overline{\mathfrak{y}} \\
\overline{\mathfrak{z}} & b & \mathfrak{x} \\
\mathfrak{y} & \overline{\mathfrak{x}} & c
\end{array}\right)
$$

with triples $(a, b, c) \in \mathbb{R}^{3}$ on the diagonal, and the nilpotent block

$$
\left(\begin{array}{lll}
0 & 0 & 0 \\
a & 0 & 0 \\
c & b & 0
\end{array}\right)
$$

represents the real dual vector space $\mathfrak{L i} \mathfrak{i}(\mathcal{N})^{*}$, where $c \in \mathbb{R}$ corresponds to an element of the one-dimensional center of the Heisenberg Lie algebra $\mathfrak{L i} \mathfrak{i}(\mathcal{N})$. Veronese coordinates for the planes over $\mathbb{R}, \mathbb{C}$, and $\mathbb{H}$ may be obtained simply by restriction to these subfields of $\mathbb{O}$.

The Jordan algebra $\mathfrak{h}_{3}(\mathbb{O})$ is commutative under the product

$$
(A, B) \rightsquigarrow \frac{1}{2}(A B+B A)
$$

and alternative but not associative. The triple of octonions $(\mathfrak{x}, \mathfrak{y}, \mathfrak{z}) \in \mathbb{O}^{3}$ admits the conjugate octonions $(\overline{\mathfrak{x}}, \overline{\mathfrak{y}}, \overline{\mathfrak{z}}) \in \mathbb{O}^{3}$ by means of the standard involution of $\mathbb{O}$, and the triality automorphism permutes the triples $(\mathfrak{x}, \mathfrak{y}, \mathfrak{z})$ and $(\overline{\mathfrak{x}}, \overline{\mathfrak{y}}, \overline{\mathfrak{z}})$ cyclically. The existence of such an automorphism actually constitutes the third order principle of spinor triality and gives rise to the general concept of ternary field.

Motivated by the linear Weingarten mapping or shape operator of the unit normal bundle of classical differential geometric surface theory, which provides the GaußKronecker curvature and the mean curvature of an oriented surface by the determinant 
and half of the trace, respectively [61], the determinant of a matrix $A \in \mathfrak{h}_{3}(\mathbb{O})$ is well defined by

$$
\operatorname{det} A=a b c-\left(a\|\mathfrak{x}\|^{2}+b\|\mathfrak{y}\|^{2}+c\|\mathfrak{z}\|^{2}\right)+2 \mathfrak{R}(\mathfrak{x} \mathfrak{y} \mathfrak{z}),
$$

although the laws of the normed division algebra $\mathbb{O}$ are non-commutative and nonassociative. It can be expressed in terms of the trace and the product via

$$
\operatorname{det} A=\frac{1}{3} \operatorname{Tr}\left(A^{3}\right)-\frac{1}{2} \operatorname{Tr}\left(A^{2}\right) \operatorname{Tr}(A)+\frac{1}{6} \operatorname{Tr}(A)^{3}
$$

where the calculations remain unspoiled by neither non-commutativity nor nonassociativity. The determinant mapping $A \rightsquigarrow \operatorname{det} A$ is a cubic form on $\mathfrak{h}_{3}(\mathbb{O})$ so there is a unique trilinear form on the triple product

$$
F: \mathfrak{h}_{3}(\mathbb{O}) \times \mathfrak{h}_{3}(\mathbb{O}) \times \mathfrak{h}_{3}(\mathbb{O}) \longrightarrow \mathbb{R}
$$

such that the identity

$$
F(A, A, A)=\operatorname{det} A
$$

holds for $A \in \mathfrak{h}_{3}(\mathbb{O})$. As a non-associative motivation ex post of the Kepplerian third spinor law $\mathrm{H}^{3}$ of planetary dynamics, the volume form $F$ gives rise to the cross product

$$
\times: \mathfrak{h}_{3}(\mathbb{O}) \times \mathfrak{h}_{3}(\mathbb{O}) \longrightarrow \mathfrak{h}_{3}(\mathbb{O})^{\star}
$$

Explicitly it is given by the trilinear assignment

$$
A \times B: C \rightsquigarrow F(A, B, C) .
$$

Despite its name, the cross product is commutative. The non-degenerate trace pairing

$$
(A, B) \rightsquigarrow \operatorname{Tr}(A B)
$$

provides the self-duality

$$
\mathfrak{h}_{3}(\mathbb{O}) \cong \mathfrak{h}_{3}(\mathbb{O})^{*}
$$

of the exceptional Jordan algebra $\mathfrak{h}_{3}(\mathbb{O})$. The duality isomorphism turns out to map points to lines and therefore to set up a bijective correspondence between points and lines of $\mathfrak{h}_{3}(\mathbb{O})$. Moreover, the self-duality provides a motivation expost of the following result of the Cayley-Dixon construction: The cross product

$$
\times: \mathfrak{h}_{3}(\mathbb{O}) \times \mathfrak{h}_{3}(\mathbb{O}) \longrightarrow \mathfrak{h}_{3}(\mathbb{O})
$$


gives rise to the induced projective geometry of the octonion projective plane or, in current terminology, Moufang plane $\mathbb{P}_{2}(\mathbb{O})$. It can be parametrized in terms of Veronese coordinates by the Hermitian matrices

$$
\left(\begin{array}{ccc}
\|\mathfrak{x}\|^{2} & \mathfrak{x} \overline{\mathfrak{y}} & \mathfrak{x} \\
\mathfrak{y} \overline{\mathfrak{x}} & \|\mathfrak{y}\|^{2} & \mathfrak{y} \\
\overline{\mathfrak{x}} & \overline{\mathfrak{y}} & 1
\end{array}\right)
$$

where

$$
\operatorname{det}\left(\begin{array}{cc}
\|\mathfrak{x}\|^{2} & \mathfrak{x} \overline{\mathfrak{y}} \\
\mathfrak{y} \overline{\mathfrak{x}} & \|\mathfrak{y}\|^{2}
\end{array}\right)=0
$$

and $(\mathfrak{x}, \mathfrak{y}) \in \mathbb{O} \times \mathbb{O}$. The embedding

$$
\mathbb{P}_{2}(\mathbb{O}) \hookrightarrow \mathbb{P}_{\mathbb{O}}\left(\mathfrak{L i e}(\mathcal{N})^{*}\right)
$$

is performed by the choice of a Veronese coordinate frame

$$
(\mathfrak{x}, \mathfrak{y}) \rightsquigarrow \mathbb{R}\left(\|\mathfrak{y}\|^{2},\|\mathfrak{x}\|^{2}, 1 ; \mathfrak{x}, \overline{\mathfrak{y}}, \mathfrak{y} \overline{\mathfrak{x}}, 1\right)
$$

which corresponds to homogeneous coordinates of the projective completion of the affine plane $\mathbb{O} \times \mathbb{O}[6,42]$. For the compact, connected manifold $\mathbb{P}_{2}(\mathbb{O})$ with an atlas of three charts, it follows

$$
\operatorname{dim}_{\mathbb{R}} \mathbb{P}_{2}(\mathbb{O})=16
$$

The lines in the non-Desarguesian projective plane $\mathbb{P}_{2}(\mathbb{O})$ are copies of $\mathbb{P}_{1}(\mathbb{O})$ and thus 8-dimensional compact spheres. For any two distinct points in $\mathbb{P}_{2}(\mathbb{O})$ there exists a unique line on which they both lie. For any two distinct lines in $\mathbb{P}_{2}(\mathbb{O})$ there is a unique point on both of them. There exists a projective duality correspondence that maps points to lines and vice versa while preserving the incidence relation. Since the space of all points lying on any given line is a copy of $\mathbb{P}_{1}(\mathbb{O})$, so is the space of all lines containing a given point. In view of the splitting

$$
\mathfrak{h}_{3}(\mathbb{O}) \cong \mathfrak{h}_{2}(\mathbb{O}) \oplus(\mathbb{O} \oplus \mathbb{O})
$$

it follows by restriction to spinors of norm one and restriction to the subfield $\mathbb{C} \hookrightarrow \mathbb{O}$ :

Theorem 10 The 4-dimensional real Jordan algebra $\mathfrak{h}_{2}(\mathbb{C})$ implements the submersion $\eta_{2}: \mathbb{S}_{3} \longrightarrow \mathbb{S}_{2}$ of the Hopf principal circle bundle $\mathbb{S}_{1} \hookrightarrow \mathbb{S}_{3} \stackrel{\eta_{2}}{\longrightarrow} \mathbb{S}_{2}$, where $\mathbb{S}_{2} \cong \mathbb{P}_{1}(\mathbb{C})$.

The Jordan algebra $\mathfrak{h}_{2}(\mathbb{O})$ is isomorphic to the $\mathbb{R}$-algebra

$$
\mathfrak{S}_{0}(\mathbb{O} \oplus \mathbb{R}) \cong \mathbb{O} \oplus \mathbb{R} \oplus \mathbb{R}
$$


where the octonion spin factor

$$
\mathfrak{S}_{0}(\mathbb{O}) \cong \mathbb{O} \oplus \mathbb{R}
$$

is invariant under the natural action of the Lorentz-Möbius group $\operatorname{PSO}(1,8, \mathbb{R})$. Notice that the Jordan algebra $\mathfrak{S}_{0}(\mathbb{O})$ is freely generated by $\mathbb{O}$ modulo the square relations

$$
\mathfrak{x}^{2}=\mathfrak{x} \mathfrak{x}=\mathfrak{x} \overline{\mathfrak{x}}=\|\mathfrak{x}\|^{2},
$$

and inherits its multiplication by polarization of the scalar product.

Scholium 2. The Jordan algebra $\mathfrak{h}_{2}\left(\mathbb{O} \hookrightarrow \mathfrak{h}_{3}(\mathbb{O})\right.$ suggests to realize the dynamic quantization by the spectral embedding of the metaplectic coadjoint orbit model of $\widehat{\mathcal{N}}$ into the exceptional Jordan algebra $\mathfrak{h}_{3}(\mathbb{O})$.

The quaternion Hopf principal sphere bundle over the compact sphere $\mathbb{S}_{4} \cong$ $\mathrm{O}(5, \mathbb{R}) / \mathrm{O}(4, \mathbb{R}) \cong \mathrm{SO}(5, \mathbb{R}) / \mathrm{SO}(4, \mathbb{R})$ or canonical quaternion line bundle reads

$$
\mathbb{S}_{3} \hookrightarrow \mathbb{S}_{7} \longrightarrow \mathbb{S}_{4}
$$

where $\mathbb{S}_{4} \cong \mathbb{P}_{1}(\mathbb{H})$. Similarly, the octonion Hopf principal sphere bundle over the compact sphere $\mathbb{S}_{8} \cong \mathrm{O}(9, \mathbb{R}) / \mathrm{O}(8, \mathbb{R}) \cong \mathrm{SO}(9, \mathbb{R}) / \mathrm{SO}(8, \mathbb{R})$ reads

$$
\mathbb{S}_{7} \hookrightarrow \mathbb{S}_{15} \longrightarrow \mathbb{S}_{8},
$$

where $\mathbb{S}_{8} \cong \mathbb{P}_{1}(\mathbb{O})$. A fiber of the Hopf submersion is the intersection of the sphere $\mathbb{S}_{15} \hookrightarrow \mathbb{R}^{16}$ with a line through the origin of the affine plane $\mathbb{O} \times \mathbb{O}$. Every such line represents an 8 -dimensional $\mathbb{R}$-linear vector subspace of $\mathbb{O} \times \mathbb{O} \cong \mathbb{R}^{16}$, and its intersection with $\mathbb{S}_{15}$ is the unit sphere of this vector subspace. Thus the fibers of the octonion Hopf submersion are given by pairwise linked 7-dimensional compact spheres inside $\mathbb{S}_{15}$.

The real dimension of the automorphism group $\operatorname{Aut}\left(\mathfrak{h}_{3}(\mathbb{O})\right)$ of the exceptional Jordan algebra $\mathfrak{h}_{3}(\mathbb{O})$ satisfies

$$
\operatorname{dim}_{\mathbb{R}} \operatorname{Aut}\left(\mathfrak{h}_{3}(\mathbb{O})\right)=\operatorname{dim}_{\mathbb{R}} \operatorname{Spin}(9, \mathbb{R})+\operatorname{dim}_{\mathbb{R}} \mathbb{P}_{2}(\mathbb{O})=36+16=52,
$$

so that the isomorphy 


$$
\operatorname{Aut}\left(\mathfrak{h}_{3}(\mathbb{O})\right) \cong \mathbb{P}_{\mathbb{O}}\left(\mathfrak{L i e}(\mathcal{N})^{*}\right) \times \mathbb{Z}_{2}
$$

holds.

\section{The open-book foliation with covariant pages as an even pure half-spinor}

In the framework of classical geometric algebra, let $\operatorname{GL}(4, \mathbb{R})$ denote the general linear group, which is a normal subgroup of the group of collineations $\Gamma L(4, \mathbb{R})$ of the exceptional real vector space $\mathbb{R}^{4}$ under its orthogonal contact geometry. The dilations and transvections with respect to the hyperplane $\mathfrak{L} \mathfrak{i}(\mathcal{N})^{*} \hookrightarrow \mathbb{R}^{4}$ form together with the identical application a subgroup $\mathcal{D}\left(\mathfrak{L i e}(\mathcal{N})^{*}\right)$ of $\operatorname{GL}(4, \mathbb{R}) \hookrightarrow \Gamma L(4, \mathbb{R})$. The transvections of the hyperplane $\mathfrak{L} \mathfrak{i} \mathfrak{e}(\mathcal{N})^{*}$ and the identical application form a normal Abelian subgroup $\mathcal{T}\left(\mathfrak{L i e}(\mathcal{N})^{*}\right) \hookrightarrow \mathcal{D}\left(\mathfrak{L} \mathfrak{i}(\mathcal{N})^{*}\right)$ which is isomorphic to the additive group $\mathfrak{L i e}(\mathcal{N})^{*} \cong \mathbb{R}^{3}$. The factor group $\mathcal{D}\left(\mathfrak{L i e}(\mathcal{N})^{*}\right) / \mathcal{T}\left(\mathfrak{L i e}(\mathcal{N})^{*}\right)$ is isomorphic to the multiplicative group $\mathbb{R}^{\times}$of the punctured line. In the complex projective manifold $\mathbb{P}_{\mathbb{C}}\left(\mathfrak{L} \mathfrak{i} \mathfrak{e}(\mathcal{N})^{*}\right)$ with the absolute plane corresponding to $\mathfrak{L i} \mathfrak{e}(\mathcal{N})^{*} \hookrightarrow \mathbb{P}_{\mathbb{C}}\left(\mathfrak{L} \mathfrak{i} \mathfrak{e}(\mathcal{N})^{*}\right)$, the group $\mathcal{D}\left(\mathfrak{L} \mathfrak{i} \mathfrak{e}(\mathcal{N})^{*}\right)$ reveals to be the group of affine transformations of $\mathbb{R}^{3} \hookrightarrow \mathbb{R}^{4}$, which transforms each affine line into a parallel line, and $\mathcal{T}\left(\mathfrak{L i e}(\mathcal{N})^{*}\right)$ identifies with the group of translations in $\mathbb{R}^{3}$. The projectivized coadjoint action $\operatorname{PCoAd}(\bullet)$ of the Heisenberg unipotent Lie group $\mathcal{N}$ cuts a horizontal line from the absoliute plane, which is orthogonal to the non-isotropic line in the spinorial gauge kernel $\mathrm{PO}^{\prime}(4, \mathbb{R}) \cong \mathrm{O}^{\prime}(4, \mathbb{R})$ containing the gyroscopic axis in $\mathbb{P}_{\mathbb{C}}\left(\mathfrak{L i e}(\mathcal{N})^{*}\right)$ of the Hopf principal circle bundle $\mathbb{S}_{1} \hookrightarrow \mathbb{S}_{3} \stackrel{\eta_{2}}{\longrightarrow} \mathbb{S}_{2}$ over the two-dimensional compact spin manifold $\mathbb{S}_{2} \cong \mathbb{P}_{1}(\mathbb{C})$.

Let $\mathrm{O}(4, \mathbb{R}) \hookrightarrow \mathrm{GL}(4, \mathbb{R})$ denote the orthogonal subgroup of the general linear group. It generates the symmetries of the complex projective manifold $\mathbb{P}_{\mathbb{C}}\left(\mathfrak{L} \mathfrak{i}(\mathcal{N})^{*}\right)$ and admits the special orthogonal group $\mathrm{SO}(4, \mathbb{R}) \hookrightarrow \mathrm{O}(4, \mathbb{R})$ of rotations of $\mathbb{R}^{4}$ as a normal subgroup of index

$$
(\mathrm{O}(4, \mathbb{R}): \mathrm{SO}(4, \mathbb{R}))=2
$$

over the field of central projective collineations $\operatorname{PSO}(1,1, \mathbb{R}) \cong \mathbb{R}$. Denote by $\mathbb{R}^{\times 2}$ the multiplicative Abelian group generated by the squares of the multiplicative group

$$
\mathbb{R}^{\times} \cong \mathcal{D}\left(\mathfrak{L} \mathfrak{i} \mathfrak{e}(\mathcal{N})^{*}\right) / \mathcal{T}\left(\mathfrak{L} \mathfrak{i} \mathfrak{e}(\mathcal{N})^{*}\right)
$$

The canonical group epimorphism of the exceptional orthogonal group onto the free Abelian Witt group WQ $(\mathbb{R})$ over the field of central projective collineations $\operatorname{PSO}(1,1, \mathbb{R}) \cong \mathbb{R}$

$$
\mathrm{O}(4, \mathbb{R}) \longrightarrow \mathbb{R}^{\times} / \mathbb{R}^{\times 2} \cong \mathrm{WS}(\mathbb{R})
$$


admits the spinorial gauge kernel $\mathrm{O}^{\prime}(4, \mathbb{R}) \hookrightarrow \mathrm{SO}(4, \mathbb{R})$. Due to the square factor of $\mathbb{R}^{\times 2}$, it restricts to the canonical spinorial norm isomorphism

$$
\text { det }: \operatorname{WS}(\mathbb{R}) \longrightarrow \mathbb{R}^{\times} / \mathbb{R}^{\times 2}
$$

of order $\left[\mathbb{R}^{\times}: \mathbb{R}^{\times 2}\right]=2$ over $\mathbb{R} \cong \operatorname{PSO}(1,1, \mathbb{R})$.

The spinorial norm isomorphism det implements the isometries belonging to the orthogonal group $\mathrm{O}(4, \mathbb{R})$ by the product of at most four symmetries of the metaplectic coadjoint orbit model associated with the quadratic form of temporal-spatial relativistic signature $(1,3)$ and Witt index 4 . In this context, symmetry means a linear representation of the Lie group $\mathrm{O}(4, \mathbb{R})$ which is an involution in the sense that it reverses a line and leaves every vector in the hyperplane orthogonal to this line fixed. And every symmetry, being an involution, it its own inverse.

The discriminant is - 1 , not a square in the real number field $\mathbb{R}(?)$. The existence of isotropic vectors affords the chain of group isomorphisms

$$
\mathrm{O}^{\prime}(4, \mathbb{R}) \cong \mathrm{PO}^{\prime}(4, \mathbb{R}) \cong \operatorname{PSL}(2, \mathbb{R}(\sqrt{-1})) \cong \operatorname{PSL}(2, \mathbb{C}) \cong \operatorname{PSO}(1,3, \mathbb{R}),
$$

and due to the underlying isotropy of the fundamental spectral dual pair (Copernicus I), the isomorphism

$$
\mathrm{SO}(4, \mathbb{R}) / \mathrm{O}^{\prime}(4, \mathbb{R}) \cong \mathbb{R}^{\times} / \mathbb{R}^{\times 2}
$$

of order 2 over $\mathbb{R} \cong \operatorname{PSO}(1,1, \mathbb{R})$.

The unitary dual group or orthogonal character group $\widehat{\mathrm{WQ}}(\mathbb{R}) \cong \mathrm{SO}(2, \mathbb{R}) \cong$ $\mathrm{U}(1, \mathbb{C}) \cong \mathbb{S}_{1}$ gives rise to the signature isomorphism

$$
\text { sign }: \mathrm{WQ}(\mathbb{R}) \longrightarrow \mathbb{Z}=\mathbb{N} \cup-\mathbb{N}
$$

onto the zooming action of the free Abelian group $(\mathbb{Z},+)$ with basis $\{1\}$. The doubling by the signature isomorphism was already known to Newton who used it for an explanation of the central body's double gravitation applied to the orbital swiveling process in accordance with the third Kepplerian spinor law $\mathrm{H}^{3}$ of the planetary dynamics of the heliocentric system and the third order principle of spinor triality.

The wideband Witt group over the field of central projective collineations $\operatorname{PSO}(1,1, \mathbb{R})$ $\cong \mathbb{R}$

$$
\mathrm{WQ}(\mathbb{R}) \cong \widehat{\widehat{\mathrm{WQ}}}(\mathbb{R}),
$$

dual to the character group $\widehat{\mathrm{WQ}}(\mathbb{R})$, consists of the isometry classes of non-degenerate symmetric bilinear forms over $\mathbb{R}$, the law of composition being the "fermionic" orthogonal sum $\perp$. Thus the dual pair

$$
\widehat{\widehat{W Q}}(\mathbb{R}), \mathrm{WQ}(\mathbb{R})) \quad(\text { Copernicus II) }
$$


arises as a structure group pair of the Kantian cosmology. The even pure spinors basically establish the connection between the concept of time in terms of the phase factor in $\mathbb{S}_{1} \cong \mathrm{SO}(2, \mathbb{R}) \cong \mathrm{U}(1, \mathbb{C})$ and the contact process of successively counting ladder rungs over the two-sided hyperbolic plane $\mathcal{O}_{\infty} \hookrightarrow \mathbb{P}_{\mathbb{C}}\left(\mathfrak{L i} \mathfrak{e}(\mathcal{N})^{*}\right)$. The dual pairs (Copernicus I) \& (Copernicus II) are in correspondence to the higher level of explanatory accomplishment of the devout Copernican Keppler by relating the metaplectic representations of the Lie group $\operatorname{Mp}(2, \mathbb{R})$ in terms of the Witt spinor norm group WS $(\mathbb{R})$ to the half-spin representations of the special Clifford group associated to $\operatorname{PSO}(1,3, \mathbb{R})[10]$ and acting on the vector spaces of odd and even pure half-spinors of $\mathbb{P}_{\mathbb{C}}\left(\mathfrak{L i e}(\mathcal{N})^{*}\right)$.

The constructive generation of WQ $(\mathbb{R})$ in accordance with Witt's cancellation theorem of the orthogonal calculus of real quadratic vector spaces bears the Genesis metaphor of two-fold Jacob's ladder in mind. Due to its integral rungs, the ladder gains access to the ordo orbium nuclear spin magnetic resonance tomographic analysis within the complex projective manifold $\mathbb{P}_{\mathbb{C}}\left(\mathfrak{L} \mathfrak{i} e(\mathcal{N})^{*}\right)$ of quantum field theory. The neutral element of WQ $(\mathbb{R})$ is the isometry class of hyperbolic vector subspaces of $\mathfrak{L} \mathfrak{i}(\mathcal{N})^{*}$ which can be conceived as a system of reference rigidly fixed to the earth. The inverse elements of WQ $(\mathbb{R})$ is defined by the isometry classes of inverse symmetric bilinear forms. The important point of orthogonal geometry is that the Witt cancellation law holds for the isometry classes of non-degenerate symmetric bilinear forms over the field of central projective collineations $\operatorname{PSO}(1,1, \mathbb{R}) \cong \mathbb{R}$ and affords the identification of the monoid arising from these isometry classes with the wideband Witt group WQ $(\mathbb{R})$ over $\mathbb{R} \cong \operatorname{PSO}(1,1, \mathbb{R})$.

An equivalent formulation of Witt's fundamental cancellation theorem, called Witt's extension theorem of the orthogonal calculus of real quadratic vector spaces, deals with the prolongation of isometries of non-singular metric vector spaces of finite dimension over a commutative field to orthogonal automorphisms. Inversive orthogonal geometry of real quadratic vector spaces then involves the zooming procedure referred to the spinor gauge supra.

Remark 7 More general, the signature isomorphism holds for all ordered fields, in which each positive element is a square. Using the the orthogonal sum as addition operation and the tensor product as multiplicative operation, WQ $(\mathbb{R})$ forms a commutative ring with unit element. It is called the Witt ring over the field of central projective collineations $\mathbb{R} \cong \operatorname{PSO}(1,1, \mathbb{R})[43]$.

Taking into account of Witt's cancellation theorem and the two orientations associated with the equatorial great circle group $\operatorname{SO}(2, \mathbb{R}) \cong \mathbb{S}_{1}$ of the supporting oriented Riemann contact sphere $\mathbb{S}_{2} \hookrightarrow \mathfrak{L} \mathfrak{i e}(\mathcal{N})^{*}$, the zooming action of the singular coadjoint orbit $\mathcal{O}_{\infty} \hookrightarrow \mathfrak{L} \mathfrak{i}(\mathcal{N})^{*}$ of $\mathcal{N}$ finds its expression in $\mathbb{P}_{\mathbb{C}}\left(\mathfrak{L i e}(\mathcal{N})^{*}\right)$ by the projective limit of metaplectic coadjoint orbits

$$
\mathcal{O}_{\infty}=\frac{1}{2} \text { proj. } \lim _{\downarrow \mathrm{WQ}(\mathbb{R})} \mathcal{O}_{\bullet}
$$

inherently projected along the axial action of the Witt spinor norm group WS $(\mathbb{R}) \cong$ $\mathbb{R}^{\times} / \mathbb{R}^{\times 2}$ of order 2 . 
Theorem 11 Supplementary to the Hopf principal circle bundle $\mathbb{S}_{1} \hookrightarrow \mathbb{S}_{3} \stackrel{\eta_{2}}{\longrightarrow} \mathbb{S}_{2}$ with Hopf submersion $\eta_{2}$, the binding $\mathbb{S}_{1} \cong \mathbb{T} \cong \widehat{\mathrm{WQ}}(\mathbb{R})$ of the open-book foliation acts on its covariant pages along the Witt ring $\mathrm{WQ}(\mathbb{R}) \cong \mathbb{Z}$ over the field of central projective collineations $\operatorname{PSO}(1,1, \mathbb{R}) \cong \mathbb{R}$ by the periodic operation of temporal unfolding.

As an application of the projective choice of angular gauge function of relativistic gravitation by the fixed star attachment of Foucault's spherical pendulum swivel at the choice of spinor gauge, integral split reduction of equivalence classes of real quadratic vector spaces under the inherent action of the Witt group WQ(R) over the field of central projective collineations $\operatorname{PSO}(1,1, \mathbb{R}) \cong \mathbb{R}$ is zooming stepwise the two-sheeted conformal anti-de-Sitter world along the stellarization ladders $\pm \mathbb{N}$ onto hyperbolic planes which are orthogonal to the gyroscopic axis of the supplementary open-book foliation in $\mathbb{P}_{\mathbb{C}}\left(\mathfrak{L} \mathfrak{i} \mathfrak{e}(\mathcal{N})^{*}\right)$ and admit radial geodesic trajectories with respect to the hyperbolic metric of the Poincaré open disc model. Its traceless symplectic matrices in $\operatorname{Sp}(2, \mathbb{R}) \cong \operatorname{SL}(2, \mathbb{R})$ or torsion elements of the module group $\operatorname{SL}(2, \mathbb{Z}) \hookrightarrow \operatorname{SL}(2, \mathbb{R})$ over the commutativer ring $\mathbb{Z}$,

$$
J=\left(\begin{array}{cc}
0 & -1 \\
+1 & 0
\end{array}\right), \quad J^{-1}={ }^{t} J=-J=\left(\begin{array}{cc}
0 & +1 \\
-1 & 0
\end{array}\right)
$$

of the two-sided homogeneous plane $\mathcal{O}_{\infty} \hookrightarrow \mathbb{P}_{\mathbb{C}}\left(\mathfrak{L} \mathfrak{i} \mathfrak{e}(\mathcal{N})^{*}\right)$ are given by the pair of commuting Fourier cotransform $\overline{\mathcal{F}}_{\mathbb{R}}$ and Fourier transform $\mathcal{F}_{\mathbb{R}}$ acting as filters on the complex Hilbert space $L^{2}(\mathbb{R})$ of square integrable signals. The group-theoretic embodiment of the filter actions is at the roots of the calculus of pseudodifferential operators and gives rise to the polarized symbol of the Schwartz kernel. It is a consequence of the first maxim of geometric quantization: Never look at the orbits of the adjoint action, rather always look at the orbits of the coadjoint action. The true reason is that the metaplectic coadjoint orbits are naturally quantized and therefore satify certain symmetry principles such as the sophisticated principle of spinor triality.

\section{Symbolic calculus on metaplectic coadjoint orbits of the $(2+$ 1)-dimensional Heisenberg unipotent Lie group $\mathcal{N} \hookrightarrow \mathrm{GL}(3, \mathbb{R})$}

The matrix $J \in \operatorname{Sp}(2, \mathbb{R})$ itself represents a planar rotation by $90^{\circ}$ counterclockwise, or a multiplication by the complex unit $i$ in the field of complex numbers $\mathbb{C}$ so that the inversion

$$
J^{-1}={ }^{t} J=-J=\left.\frac{1}{\pi} \frac{\mathrm{d}}{\mathrm{d} t}\right|_{t=0} \exp (\pi J t)
$$

represents the spin echo-stabilizing infinitesimal generator of the Hopf circular fiber $\mathbb{S}_{1} \cong \mathrm{SO}(2, \mathbb{R}) \cong \mathrm{U}(1, \mathbb{C}) \cong \widehat{\mathrm{WQ}}(\mathbb{R})$. In the context of the $\mathbb{Z}_{2}$-twist $J \rightsquigarrow-J$, the mapping exp is the exponential diffeomorphism from $\mathcal{O}_{1} \hookrightarrow \mathfrak{L i e}(\mathcal{N})^{*}$ to the Heisenberg unipotent Lie group $\mathcal{N}$. Interpreted as an $\mathbb{R}$-linear transformation $J \in$ 
$\operatorname{SL}(2, \mathbb{R})$, it defines a complex structure on the metaplectic coadjoint orbit $\mathcal{O}_{1} \in$ $\mathfrak{L i} \mathfrak{e}(\mathcal{N})^{*} / \operatorname{CoAd}(\mathcal{N})$ :

$$
\left(\mathcal{O}_{1}, J\right) \cong \mathcal{O}_{1} \otimes_{\mathbb{R}} \mathbb{C}=\mathcal{O}_{\mathbb{C}}
$$

The complexification implies the extension of symplectic groups

$$
\operatorname{SL}(2, \mathbb{R}) \cong \operatorname{Sp}\left(\mathcal{O}_{1}\right) \hookrightarrow \operatorname{Sp}\left(\mathcal{O}_{\mathbb{C}}\right) \cong \operatorname{SL}(2, \mathbb{C})
$$

It is this aspect which is of importance for the complexification of the metaplectic coadjoint orbits of $\mathcal{N}$ within the projective manifold $\mathbb{P}_{\mathbb{C}}\left(\mathfrak{L i e}(\mathcal{N})^{*}\right)$. The standard metaplectic form of the planar coadjoint orbit $\mathcal{O}_{1} \in \mathfrak{L} \mathfrak{i} e(\mathcal{N})^{*} / \operatorname{CoAd}(\mathcal{N}) \operatorname{reads}$

$$
\left(w, w^{\prime}\right) \rightsquigarrow\left(w \mid J w^{\prime}\right) .
$$

The concept of twisted convolution may be regarded as an integrated version of a twisted translation, which is derived from ordinary translation on the $(2+1)$ dimensional Heisenberg unipotent Lie group $\mathcal{N}$. It defines a structure of associative algebra on the complex Schwartz space $\mathcal{S}_{\mathbb{C}}\left(\mathcal{O}_{1}\right)$, and by the symplectic Fourier transform of order 2 instead of order 4, the Weyl calculus of isotropic symbol [37]. Because the symplectic Fourier transform refers to Lebesgue meaure $\frac{1}{2} \mathrm{~d} w$ on $\mathcal{O}_{1}$, which is selfdual with respect to the symplectic form, the polarized symbol and the isotropic symbol of the theory of pseudodifferential operators are quite closely related, differing only by normalizations. The ensuing Weyl transform

$$
\mathcal{S}_{\mathbb{C}}\left(\mathcal{O}_{1}\right) \longrightarrow \mathcal{S}_{\mathbb{C}}(\mathbb{R} \oplus \mathbb{R})
$$

which represents an isomorphism of complex algebras with involution, takes the twisted convolution product onto the composition of integral operators. The bundletheoretic construction process of unitarily induced linear representations affords the following statement on the process of dynamic quantization:

Theorem 12 The open-book foliation with covariant pages and $\mathbb{S}_{1} \cong \mathbb{T}$-binding, driven by the symplectic Fourier tranform of order two, attaches a complex Hilbert bundle with square integrable cross sections of finite energy on which the equivalence classes of unitary linear representations, which are constituting the unitary dual $\widehat{\mathcal{N}}$ of the Heisenberg unipotent Lie group $\mathcal{N}$, are realized; in fact $\mathcal{N}$ moves by rotations the fibers of the Hilbert bundle around the central axis $\mathcal{Z} \hookrightarrow \mathcal{N}$ and real Lie algebra center $\mathfrak{L} \mathfrak{i} \mathfrak{e}(\mathcal{Z}) \hookrightarrow \mathfrak{L} \mathfrak{i} \mathfrak{e}(\mathcal{N})$.

It is known that a finite dinensional quadratic space admitting a matrix with a zero on the diagonal is isotropic [43]. The direct sum of orthogonal planar hyperbolic generators

$$
\left\langle\left(\begin{array}{ll}
0 & 1 \\
1 & 0
\end{array}\right)\right\rangle \oplus\left\langle\left(\begin{array}{cc}
0 & -1 \\
-1 & 0
\end{array}\right)\right\rangle^{\perp}
$$


with isotropic coordinate axes in the two-sided hyperbolic plane $\mathcal{O}_{\infty}$ of discriminant - 1 shows that these axes are actually the only isotropic axes in $\mathcal{O}_{\infty} \hookrightarrow \mathbb{P}_{\mathbb{C}}\left(\mathfrak{L i e}(\mathcal{N})^{*}\right)$. The corresponding one-dimensional characters of the $(2+1)$-dimensional real nilpotent Heisenberg Lie algebra $\mathfrak{L} \mathfrak{i}(\mathcal{N})$ form the characteristic gyroscopic square in $\mathcal{O}_{\infty}$, which is invariant under the action of the non-cyclic Klein four-group $\mathfrak{V}_{4}$ of pure half-spinors.

All hyperbolic planes have discriminant - 1. They give rise to the free Abelian Witt group $\mathrm{WQ}(\mathbb{R})$ over the field of central projective collineations $\operatorname{PSO}(1,1, \mathbb{R}) \cong \mathbb{R}$ which is isomorphic to the additive group $(\mathbb{Z},+)$ with basis $\{1\}$. The unitary dual group $\widehat{\mathrm{WQ}}(\mathbb{R}) \cong \mathbb{S}_{1}$ of $\mathrm{WQ}(\mathbb{R})$ generates via the gyroscopic contact torus of revolution $\mathbb{S}_{1} \times \mathbb{S}_{1} \cong \mathbb{R}^{\times 2}$ the supplementary open-book foliation. Finally, it affords the Sagnac spherical velocity-cone calibration of the parallel transport facility of laser ring gyroscopes inside hyperbolic contact planes in $\mathbb{P}_{\mathbb{C}}\left(\mathfrak{L} \mathfrak{i} \mathfrak{e}(\mathcal{N})^{*}\right)$. Sagnac's effect of two counterpropagating cavity resonance modes in the two-sided hyperbolic plane $\mathcal{O}_{\infty}$ defines the origin of all laser optic gyroscope technologies.

To transform the language of Copernicus's opus magnum De revolutionibus... into the terminology of contact topology, the ordo orbium retains the time scale as the Copernican ordering criterion of the projective stellarization of the spinning earth. Relativistic timing analysis of the spinor gauge yields by the action of the non-Abelian simple Lorentz-Möbius Lie group $\operatorname{PSO}(1,3, \mathbb{R})$ on the supplementary open-book foliation by Poisson cumulative superposition.

Theorem 13 The periodicity of axial rotation granted to the action of the Witt ring $\mathrm{WQ}(\mathbb{R})$ in $\mathbb{P}_{\mathbb{C}}\left(\mathfrak{L i e}(\mathcal{N})^{*}\right)$ establishes the existence of relativistic gravitational wavelets and the orthogonal wave front sets of the relativistic gravitational wave packet emission.

A filter bank detectable radio astrophysicalization of the Witt ring $\mathrm{WQ}(\mathbb{R})$ is performed by the high precision pulse times-of-arrival data sets acquired by radio pulsar telescopes $[26,27]$. It was Kant again who predicted tellingly the generation of gravitational wave packets by energy loss in terms of "the process that spreads in concentric waves and disorganizes the same world-material over and over again."

\section{Pulsar astrophysics: the half-spin down luminosity spectrum}

As a pulsar astrophysical application, the recording of the post-Kepplerian driving spinor warping within the open-book foliation, supplementary to the inversive geometry of the Kepplerian planetary contact holonomy, deals with the relativistic measurement protocol of the cumulative period shift of periastron time in seconds. The protocol of about 40 years of observations is based on relativistic gravitational radiation damping causing orbital decay by the loss of energy, known as the half-spin down luminosity. The underlying geometry of parallel transport dynamics forms the link to the paradigmatic Foucault spherical pendulum experiment.

The relativistic gravitational radiation emission manifests in terms of the accumulating shift in epoch of periastron, and in accordance with the third order principle of 
spinor triality in terms of Veronese coordinates of a smooth surface within the projective manifold which is contained in the octonion manifold $\mathbb{P}_{\mathbb{O}}\left(\mathfrak{L i e}(\mathcal{N})^{*}\right) \cong \mathfrak{h}_{3}(\mathbb{O})$ by admittance of the absolute plane as a supporting tangent plane. It illustrates the generation of the cumulative periastron shift of the relativistic binary radio pulsar system PSR B1913+16, while the Arecibo timing measurements are marked by data points, with error bars mostly too small to seen. The high quality observation data provide conclusive and most elegant evidence for the existence of relativistic gravitational wave packet emission. The several-year hiatus of the regular timing and post-Kepplerian parameter measurements is due to the various Arecibo radio telescope upgrading projects and which have been interpolated by use of a wideband filter bank [73].

The relativistic gravitational wave packets encode the ordo orbium by the action of the free Abelian group $\mathrm{WQ}(\mathbb{R}) \cong \mathbb{Z}$. The unitary dual group $\widehat{\mathrm{WQ}}(\mathbb{R}) \cong \cong \mathbb{T} \cong \mathbb{S}_{1}$ identifies the spinor gauge of relativistic gravitation by the resonance tomographic phase imaging facility of the supplementary open-book foliation of $\mathbb{P}_{\mathbb{C}}\left(\mathfrak{L i \mathfrak { e }}(\mathcal{N})^{*}\right)$. High image resolution in radio astrophysical validation has demonstrated the energy loss by orbital shrinkage of the relativistic binary radio pulsar system PSR B1913+16 [46]. Using the Arecibo Observatory $305 \mathrm{~m}$ antenna at $18^{\circ}, 20^{\prime}, 39^{\prime \prime} \mathrm{N}, 66^{\circ}, 45^{\prime}, 10^{\prime \prime}$ $\mathrm{W}$, its pulsed radio emission was discovered in the constellation Aquila at a distance of 20870 light years by Russell Alan Hulse and Joseph Hooton Taylor, Jr. in 1974. Their discovery of the binary system and ordo orbium nuclear spin magnetic resonance tomographic analysis of it earned them the 1993 Nobel prize in physics "for the discovery of a new type of pulsar, a discovery that has opened up new possibilities for the study of gravitation."

It is known that radio pulsars are highly magnetized, spinning neutron stars which emit a narrow radio beam along the magnetic dipole axis. The weak radio source of relativistic binary radio pulsar system PSR B1913+16 consists of two neutron stars, one is an observed pular, orbiting in a very tight, highly eccentric orbit. It is to be noted that in such a binary star system, observed from a reference frame where one of the stars is at rest phase position, the secondary star moves in an elliptical orbit. The point of closest approach between the two stars is called the periastron. Equivalently, the secondary star is at periastron when the two stars admit their smallest separation. It follows that an application of the nuclear spin magnetic resonance tomographic phase imaging modality of non-invasive ultra-high field nuclear spin magnetic resonance tomography $[44,74]$ provides the high image resolution appropriate for recording the post-Kepplerian driving spinor warping. The phase controlled records on the covariant pages of the supplementary open-book foliation in $\mathbb{P}_{\mathbb{C}}\left(\mathfrak{L} \mathfrak{i} \mathfrak{e}(\mathcal{N})^{*}\right)$ are caused by the tomographic slices as planar axial cross-sections of the four-dimensional doublepoint free ruled quadric without center, consisting of accumulating shifts in the epoch diagram of periastron. To justify this statement, it needs to examine the Veronese vectors

$$
\mathbb{R}\left(\|\mathfrak{y}\|^{2},\|\mathfrak{x}\|^{2}, 1 ; \mathfrak{x}, \overline{\mathfrak{y}}, \mathfrak{y} \overline{\mathfrak{x}}\right)
$$

of points and 


$$
\left(1,\|\mathfrak{u}\|^{2},\|\mathfrak{v}\|^{2} ; \overline{\mathfrak{u}} \mathfrak{v},-\overline{\mathfrak{v}},-\mathfrak{u}\right)^{\perp}
$$

of lines in the projective manifold $\mathbb{P}_{\mathbb{C}}\left(\mathfrak{L} \mathfrak{i} \mathfrak{e}(\mathcal{N})^{*}\right)$, respectively, which arise by restriction downstairs the Cayley-Dickson construction to the rank four configuration of the classical Hopf fibration. Inspection of the Veronese vectors gives rise to the following

Theorem 14 The sequence of epochs of the half-spin down luminosity spectrum of rank four over $\mathbb{R}$ are generated via parallel transport dynamics by the ruled quadric of a hyperbolic paraboloid. It represents a real projective quadric of rank four inside the four-dimensional affine vector space $\mathbb{R}^{(1,3)}$ and a real projective quadric of rank two on the two-dimensional affine plane at infinity, in conjunction with a real projective quadric with two positive eigenvalues, and a real projective quadric with one positive eigenvalue at the two-dimensional affine plane at infinity. Its contact point with the tangent plane at infinity of the projective space $\mathbb{P}_{\mathbb{R}}\left(\mathfrak{L} \mathfrak{i}(\mathcal{N})^{*}\right) \cong \mathbb{P}_{3}(\mathbb{R})$ is the central pole $\infty \in \mathbb{P}_{\mathbb{R}}(\mathfrak{L i} \mathfrak{i}(\mathcal{Z}))$.

Scholium 3. The classification of real projective quadrics of $1 \leq \operatorname{rank} \leq 4$ in the space $\mathbb{P}_{3}(\mathbb{R})$ comprises exactly 17 prototypes modulo affine equivalences. The hyperbolic paraboloid has maximal rank four and is shift invariant due to the skew ruling configuration of parallel translation surfaces in $\mathbb{P}_{3}(\mathbb{R})$. The spectral dual pair of real Lie groups (Copernicus I) supra along attributed quantum channels supports the post-Kepplerian theorem of Alexandrov-Ovchinnikova of relativity theorem [1,2].

The Veronese coordinatization implies the following non-holonomy result.

Corollary The half-spin down luminosity spectrum is tomographically acyclic in the sense that no real plane cuts in a circle.

Like other pulsar systems, the relativistic binary radio pulsar PSR B1913+16 emits short, periodic bursts of radio emission which emulate the ticks of a high-precision clock. Its radial velocity varies from a minimum of $75 \mathrm{~km} / \mathrm{sec}$ to a maximum of 400 $\mathrm{km} / \mathrm{sec}$. The orbit is elliptical with a large eccentricity of about 0.617 , a major axis of only 6.4 light seconds, and a minor axis of 5 light seconds. It is oriented such that periastron occurs nearly perpendicular to the line of sight to the orbiting radio pulsar. Unfortunately, the line of sight passes through a complex region of the Galaxy, and due to the phenomenon of gravitational lensing one has to be very careful, when derives the value of the distance to the pulsar from the dispersion measure.

For the prototype relativistic system PSR B1913+16, the binary radio pulsar's orbital period of $7 \frac{3}{4} \mathrm{~h}$ is remarkably short. The pulsar orbit has been fully specified up to a rotation about the line of sight by filter bank techniques and signal averagers. The most recently gathered, high quality observation data have achieved $13 \mu$ s timeof-arrival measurements in each of four $100 \mathrm{MHz}$ bands, using 5 minute integrations [71-73].

Theorem 15 The inherent axial action of the wideband Witt group WQ(R) over the field of central projective collineations $\operatorname{PSO}(1,1, \mathbb{R}) \cong \mathbb{R}$ at a projective choice of spinor gauge of relativistic gravitation affords a spectral splitting $\perp$ of the spectrum $\widehat{\mathcal{N}}$ of the Heisenberg unipotent Lie group $\mathcal{N}$ into orthogonal hyperbolic planes of $\mathbb{P}_{\mathbb{C}}\left(\mathfrak{L i e}(\mathcal{N})^{*}\right)$. 
It is known from geometric algebra that every hyperbolic plane is universal. Therefore the Copernican principle of projective analysis is able by the Witt group WQ $(\mathbb{R})$ over the field of central projective collineations $\operatorname{PSO}(1,1, \mathbb{R}) \cong \mathbb{R}$ to iteratively create fields of quantum states.

\section{The de Coriolis alternating trilinear form of the ratio orbium}

The natural metaplectic structure of the generic coadjoint orbit $\mathcal{O}_{\bullet} \in \mathfrak{L i e}(\mathcal{N})^{*} / \operatorname{CoAd}$ $(\mathcal{N})$ with traceless matrix $J^{-1}={ }^{t} J=-J=\left(\begin{array}{cc}0 & 1 \\ -1 & 0\end{array}\right)$ defines the standard exterior or wedge product $\wedge$ of the exterior plane square $\bigwedge^{2}\left(\mathcal{O}_{\bullet}\right) \hookrightarrow \wedge\left(\mathcal{O}_{\bullet}\right)$ over $\mathbb{R}$. The realvalued alternating bilinear forms on the Cartesian square $\mathcal{O}_{\bullet} \times \mathcal{O}_{\bullet}$ constitute the real line $\bigwedge^{2}\left(\mathcal{O}_{\bullet}\right)$ and are uniquely determined up to a factor $\pm 1 \in \mathbb{Z}_{2}$ by every orthonormal dual basis of $\mathcal{O}_{\bullet} \hookrightarrow \mathfrak{L} \mathfrak{i}(\mathcal{N})^{*}$. The exterior product is associated by harmonic analysis on the Heisenberg unipotent Lie group $\mathcal{N}$ to the Fourier cotransform $\overline{\mathcal{F}}_{\mathbb{R}}$ with filter action on the complex Hilbert space $L^{2}(\mathbb{R})$ of square integrable signals.

For an application to the non-invasive imaging modality of nuclear spin magnetic resonance tomography, the real-valued alternating or anti-symmetric trilinear forms on the Cartesian triple product $\mathfrak{L} \mathfrak{i} \mathfrak{e}(\mathcal{N})^{*} \times \mathfrak{L} \mathfrak{i} \mathfrak{e}(\mathcal{N})^{*} \times \mathfrak{L} \mathfrak{i}(\mathcal{N})^{*}$ of the three-dimensional oriented real dual vector space $\mathfrak{L} \mathfrak{i}(\mathcal{N})^{*}$ constitute the singletons

$$
\bigwedge^{3} \mathfrak{L i e}(\mathcal{N})^{*} \cong \mathbb{R}^{\circ} \hookrightarrow \mathbb{R}
$$

of oriented voxel forms on $\Im(\mathbb{H})$ over $\mathbb{R}$, and are uniquely determined up to a factor $\pm 1 \in \mathbb{Z}_{2}$ by every orthonormal frame of $\mathfrak{L i e}(\mathcal{N})^{*}$. It gives rise to the vector product $\bar{\wedge}$ by the trilinear extension of the wedge product $\wedge$ of real-valued biforms of the exterior square $\Lambda^{2}\left(\mathcal{O}_{\bullet}\right) \hookrightarrow \bigwedge\left(\mathcal{O}_{\bullet}\right)$. It is known that the notion of vector product $\bar{\wedge}$ of two real-valued trilinear forms of $\bigwedge\left(\mathfrak{L i e}(\mathcal{N})^{*}\right)$ is $\operatorname{PSO}(3, \mathbb{R})$-specific in the sense that it cannot be defined in the vector spaces with dimensions other than three or those admitting relativistic temporal-spatial signature $(1,3)$. In terms of the Lie bracket of the three-dimensional real Lie algebra $\Im(\mathbb{H})$ of the normed division algebra of real quaternions, it follows for the vector product. The oriented volume form allows to deal with inertial force fields. A direct extension of the volume form from $\Im(\mathbb{H})$ to the non-associative alternative normed division algebra of octonions $\mathbb{O}$ is not possible, because $\Im(\mathbb{O})$ reveals to be not a Lie algebra.

One of its first physical descriptions of inertial force fields can be traced back to the work of Gaspard Gustave de Coriolis (1792-1843). It follows for the geodesic curvature in terms of the de Coriolis alternating trilinear form:

Theorem 16 Let $\gamma$ denote the unit-velocity parametrization of a smooth loop in the two-dimensional compact spin manifold $\mathbb{S}_{2} \hookrightarrow \mathbb{P}_{\mathbb{C}}\left(\mathfrak{L} \mathfrak{i}(\mathcal{N})^{*}\right)$, then the geodesic curvature form $k_{\gamma}$ with input parameter on the time scale reads in terms of the oriented 
real duality $\left(\mathfrak{L i e}(\mathcal{N})^{*}, \mathfrak{L i e}(\mathcal{N})\right)$

$$
k_{\gamma}: t \rightsquigarrow\left(\gamma(t) \bar{\wedge} \gamma^{\prime}(t) \mid \gamma^{\prime \prime}(t)\right)
$$

with respect to the outward-pointing orientation of the Riemann contact sphere $\mathbb{S}_{2} \hookrightarrow$ $\mathbb{P}_{\mathbb{C}}\left(\mathfrak{L i e}(\mathcal{N})^{*}\right)$.

The de Coriolis alternating trilinear form gives rise to a measurement of the signed lengths of the tangential acceleration vectors and therefore to the de Coriolis inertial force field associated to the smooth loop $\gamma$ in the supporting oriented Riemann contact sphere $\mathbb{S}_{2} \hookrightarrow \mathbb{P}_{\mathbb{C}}\left(\mathfrak{L i e}(\mathcal{N})^{*}\right)$.

Triality backstage inspection. The alternating trilinear form $k_{\gamma}$ of geodesic curvature suggests to look at the symmetrization of odd pure half-spinors in $\mathbb{P}_{\mathbb{C}}\left(\mathfrak{L i e}(\mathcal{N})^{*}\right)$ by the action of the Witt spinor norm group WS $(\mathbb{R})$ in order to replace $k_{\gamma}$ by the symmetric trilinear form $\Phi$ of the third order principle of spinor triality. The sophisticated triality principle of the classical algebraic theory of spinors and associative Clifford/Graßmann algebra models over $\Phi$ was discovered by Élie Cartan and made more transparent Chevalley in 1954 along the Cayly-Dickson duplication strategy [10]. The third order principle of spinor triality is concerned with the spherical contact geometric calibration $\frac{3}{2}$-power ratio orbium

$$
\frac{\text { cubic determinant form }}{\text { quadratic form }}
$$

(Keppler III)

where the numerator form on $\mathbb{P}_{\mathbb{O}}\left(\mathfrak{L i e}(\mathcal{N})^{*}\right)$, the denominator form $\omega$ on $\mathbb{P}_{\mathbb{O}}\left(\mathfrak{L i e}(\mathcal{N})^{*}\right)$, the symmetric trilinear form $\Phi$ on the triple product space $\mathbb{P}_{\mathbb{O}}\left(\mathfrak{L i e}(\mathcal{N})^{*}\right) \times$ $\mathbb{P}_{\mathbb{O}}\left(\mathfrak{L i e}(\mathcal{N})^{*}\right) \times \mathbb{P}_{\mathbb{O}}\left(\mathfrak{L i e}(\mathcal{N})^{*}\right)$ are left invariant under the action of the real vector space automorphism the non-associative alternative normed division algebra of octonions $\mathbb{O} \hookrightarrow \mathbb{P}_{\mathbb{O}}\left(\mathfrak{L i e}(\mathcal{N})^{*}\right)$. The faithful spin representation $\rho$ on $\mathfrak{S}$ gives rise to the construction of a real vector space automorphism of the octonion extension $\mathfrak{L i e}(\mathcal{N})^{*} \otimes_{\mathbb{R}} \mathbb{O}$ or equivalently of an $\mathbb{R}$-linear projectivity $j$ of order 3 of the real vector space $\mathbb{P}_{\mathbb{O}}\left(\mathfrak{L i e}(\mathcal{N})^{*}\right)$. The point is that $\Phi$ endowes $\mathbb{P}_{\mathbb{O}}\left(\mathfrak{L i e}(\mathcal{N})^{*}\right)$ with a nonassociative and commutative real algebra structure [10]. It is to be noted that the a real vector space automorphism $j$ permutes cyclically the two isomorphic subspaces of odd and even pure half-spinors of the split vector space of spinors $\mathfrak{S}=\mathfrak{S}_{-} \oplus \mathfrak{S}_{+}$ for the Witt index 4 so that the series of interchanges

$$
j^{-1}(\mathbb{O})=\mathfrak{S}_{-}, \quad j^{-1}\left(\mathfrak{S}_{-}\right)=\mathfrak{S}_{+}, \quad j^{-1}\left(\mathfrak{S}_{+}\right)=\mathbb{O}
$$

holds. Both pure half-spinor spaces admit the same dimension $8=\operatorname{dim}_{\mathbb{R}} \mathfrak{S}_{-}=$ $\operatorname{dim}_{\mathbb{R}} \mathfrak{S}_{+}=\operatorname{dim}_{\mathbb{R}} \mathbb{O}$ of the non-associative alternative normed division algebra of octonions $\mathbb{O}$ under its Cayley-Dickson duplication construction. The conjugate of any one of the non-isotropic vector spaces

$$
\left\{\mathfrak{S}_{-}, \mathfrak{S}_{+}, \mathbb{O}\right\}
$$


is the direct sum of the other one [6]. It is to be noted that the existence of such a real vector space automorphism of order 3 , which actually is a swiveling algebra automorphism, sets the backstage for the third order principle of spinor triality, in conjunction with the identification of $\mathbb{O}$ with the eight-dimensional real vector space $\mathfrak{S}_{\text {- }}$ of odd pure half-spinors under a second order automorphism of $\mathbb{P}_{\mathbb{O}}\left(\mathfrak{L i e}(\mathcal{N})^{*}\right)$ which leaves the cubic form as well as the quadratic form $\omega$ invariant. The ratio (Keppler III) permits to derive the third Kepplerian spinor law $\mathrm{H}^{3}$ of planetary dynamics. Since trialities only occur in dimensions $3 \cdot 2^{m}$, where $m \in\{0,1,2,3\}$ due to the Cayley-Dickson duplication strategy, spinors and the invariants (Casimir) of the third order principle of spinor triality are capable to emphasize the profoundness of the pure half-spinor approach to the dynamics of Kepplerian planetary motion and its visualization by the Foucault's spherical pendulum experiment of dual contact holonomy which need to go deeply into the structure of the projective manifold on which they are defined.

\section{Covariance of the global angular gauge function}

The canonical spinorial norm isoomorphism $\mathrm{SO}(4, \mathbb{R}) \longrightarrow \mathrm{WS}(\mathbb{R})$ on the metaplectic open-book foliation which is supplementary to the Hopf principal circle bundle $\mathbb{S}_{1} \hookrightarrow \mathbb{S}_{3} \stackrel{\eta_{2}}{\longrightarrow} \mathbb{S}_{2}$ in the complexification $\mathbb{P}_{\mathbb{C}}\left(\mathfrak{L i e}(\mathcal{N})^{*}\right)$. The binding of the open-book foliation with covariant pages is given by the typical circular fiber $\mathbb{S}_{1} \cong \operatorname{SO}(2, \mathbb{R})$ of the Hopf fibration. The covariant action of the Witt spinor norm group WS $(\mathbb{R}) \cong \mathbb{R}^{\times} / \mathbb{R}^{\times 2}$ on the spin echo-stabilized, symplectic swing-planes of the Foucault spherical pendulum holonomy experiment can be horizontally parametrized in terms of the real-valued angular gauge function $t \rightsquigarrow \theta(t)$ and a smooth unit-velocity loop $\gamma$ in the supporting oriented Riemann contact sphere $\mathbb{S}_{2} \hookrightarrow \mathbb{P}_{\mathbb{C}}\left(\mathfrak{L i e}(\mathcal{N})^{*}\right)$ by the field of velocity vectors

$$
v: t \rightsquigarrow \cos \theta(t) \gamma^{\prime}(t)+\sin \theta(t) J \gamma^{\prime}(t)
$$

and the symplectic structure of the two-sided homogeneous plane $\mathcal{O}_{\infty} \hookrightarrow \mathbb{P}_{\mathbb{C}}$ $\left(\mathfrak{L i e}(\mathcal{N})^{*}\right)$. Then $v$ is a unit-length vector field around the loop $\gamma$.

Theorem 17 The spinor gauge parametrization by the real-valued angular gauge function $t \rightsquigarrow \theta(t)$ categorizes parallelity in the sense that for every parallel vector field $t \rightsquigarrow v(t)$ around the smooth unit-velocity loop $\gamma$ in the two-dimensional compact spin manifold $\mathbb{S}_{2}$ the functional equation

$$
\gamma^{\prime}(t)=\cos \left(\theta(t)+\theta_{0}\right) v(t)+\sin \left(\theta(t)+\theta_{0}\right) J v(t)
$$

holds, where $\theta_{0} \in[0,2 \pi[$ denotes a phase shift.

In terms of the geodesic curvature function $k_{\gamma}$ of the loop $\gamma$, it follows for the symplectic structure $J$ and the covariant derivative of the transposed symplectic structure ${ }^{t} J$ applied to the projection of $t \rightsquigarrow v^{\prime}(t)$ onto the two-sided homogeneous plane $\mathcal{O}_{\infty} \hookrightarrow \mathbb{P}_{\mathbb{C}}\left(\mathfrak{L i e}(\mathcal{N})^{*}\right):$ 
Theorem 18 The covariance action of the metaplectic Lie group $\mathrm{Mp}(2, \mathbb{R})$ implies the identities

$$
\nabla \gamma^{\prime}=k_{\gamma} J v, \quad \nabla^{t} J v=k_{\gamma} \gamma^{\prime}
$$

for the unit-length parallel vector field $t \rightsquigarrow v(t)$ around the unit-velocity loop $\gamma$ with derivative $\gamma^{\prime}$.

Due to the metaplectic correspondence, the relation of the reduced covariance identities to the Fourier cotransform $\overline{\mathcal{F}}_{\mathbb{R}}$ and the Fourier transform $\mathcal{F}_{\mathbb{R}}$ over the field of central projective collineations $\operatorname{PSO}(1,1, \mathbb{R}) \cong \mathbb{R}$ becomes obvious through projection along the Witt group WQ $(\mathbb{R})$ onto the singular coadjoint orbit $\mathcal{O}_{\infty} \hookrightarrow$ $\mathbb{P}_{\mathbb{C}}\left(\mathfrak{L} \mathfrak{i}(\mathcal{N})^{*}\right)$ of the unipotent Heisenberg Lie group $\mathcal{N}$.

Theorem 19 Due to the covariant action of the Witt group WQ(R) over $\mathbb{R} \cong$ $\operatorname{PSO}(1,1, \mathbb{R})$, it is the real-valued angular gauge function $t \rightsquigarrow \theta(t)$ on which the spinor gauge approach of the Foucault spherical pendulum holonomy is based. If $k_{\gamma}$ denotes the geodesic curvature of the smooth loop $\gamma$, the global angular gauge function admits the temporal representation

$$
\theta: t \rightsquigarrow \theta_{0}-\int_{t_{0}}^{t} k_{\gamma}(s) \mathrm{d} s
$$

where $\theta_{0} \in \mathbb{R}$ indicates the initial position of the symplectic swing-plane at time $t_{0} \in \mathbb{R}$.

Thus the derivative $\theta^{\prime}$ of the real-valued angular gauge function $t \rightsquigarrow \theta(t)$ is the skew-derivative of the geodesic curvature $k_{\gamma}$. It ensures that for every unit-length parallel vector field $v$ around the loop $\gamma$ the derivative $\gamma^{\prime}$ with an approriate phase shift $\theta_{0} \in\left[0,2 \pi\right.$ [ included, admits the covariant derivative $\nabla \gamma^{\prime}=0$ [61]. The geodesics $\gamma$ on the two-dimensional oriented smooth manifold $\mathcal{M}=\mathbb{S}_{2} \hookrightarrow \mathbb{P}_{\mathbb{C}}\left(\mathfrak{L i e}(\mathcal{N})^{*}\right)$ are great circles, which means the intersection of the two-dimensional compact spin manifold $\mathbb{S}_{2}$ with a plane through the origin. For the equatorial great circle, this plane is $\mathcal{O}_{\infty}$ at latitude direction angle $\varphi=\frac{1}{2} \pi$. Then $k_{\gamma}=0$ implies that the angular gauge function $t \rightsquigarrow \theta(t)$ is a constant function. In particular, the derivative $\gamma^{\prime}$ defines a parallel vector field around the loop $\gamma$ on the two-dimensional compact spin manifold $\mathbb{S}_{2} \cong \mathbb{P}_{1}(\mathbb{C})$.

\section{Calibrated contact geometry and spherical holonomy}

The supplementary open-book foliation gives rise to the phase framing gyroscopic contact torus of revolution $\mathbb{S}_{1} \times \mathbb{S}_{1}$ of cross-sectional discs contained in the spin echo-stabilized, symplectic swing-plane through the initial axis and the central circle of nutation over the radially scaled, complementary disc is fibered by pairs of $\varphi$ inclined Villarceau circular fibers of opposite orientations at the interface of quantum 
field theory of the Heisenberg unipotent Lie group $\mathcal{N}$ with nilpotent Heisenberg Lie algebra $\mathfrak{L} \mathfrak{i}(\mathcal{N})$, and system relativity. A cross-sectional disc for the corresponding solid torus meets every Villarceau circular fiber inside the flat torus just once. The complementary disc meets each fiber outside the torus once. The circular fibers on the torus meet each disc once in its bounding circle and provide a diffeomorphism between these two circles. Thus the manifold of fibers is made up of two discs glued together by a diffeomorphism of their boundaries, which is just the two-dimensional compact homogeneous spin manifold $\mathbb{S}_{2} \cong \mathrm{SO}(3, \mathbb{R}) / \mathrm{SO}(2, \mathbb{R}) \cong \mathbb{P}_{1}(\mathbb{C})$.

The canonical spherical coordinate chart

$$
\sigma:] 0,2 \pi[\times] 0, \pi\left[\longrightarrow \mathbb{S}_{2}\right.
$$

of the supporting oriented Riemann contact sphere $\mathbb{S}_{2} \cong \operatorname{SO}(3, \mathbb{R}) / \mathrm{SO}(2, \mathbb{R})$ at a point $(\theta, \varphi) \in] 0,2 \pi[\times] 0, \pi[$ reads

$$
\sigma:(\theta, \varphi) \rightsquigarrow(\sin \varphi \cos \theta, \sin \varphi \sin \theta, \cos \varphi) .
$$

The image of the chart $\sigma$ attains all points of the two-dimensional compact spin manifold $\mathbb{S}_{2}$ except the north and south poles which are the opposite contact points of the metaplectic coadjoint orbits in the real dual vector space $\mathfrak{L i e}(\mathcal{N})^{*}$, and the spin-echo meridian between them along the $\varphi$-latitudinal direction angle $\theta=0$ of the real-valued angular gauge function $t \rightsquigarrow \theta(t)$ of the spin echo-stabilized, symplectic swing-plane of the Foucault spherical pendulum holonomy experiment. The Jacobian matrix

$$
\mathrm{D} \sigma=\left(\begin{array}{cc}
-\sin \varphi \sin \theta & \cos \varphi \cos \theta \\
\sin \varphi \cos \theta & \cos \varphi \sin \theta \\
0 & -\sin \varphi
\end{array}\right)
$$

associated to the differential $\mathrm{D} \sigma \in \mathfrak{L} \mathfrak{i}(\mathrm{SO}(3, \mathbb{R}))$ has rank two. The vector product $\sigma_{\theta} \bar{\wedge} \sigma_{\varphi}$ of the two orthogonal column vectors, which constitute the differential $\mathrm{D} \sigma$, yields the extended alternating trilinear identity

$$
\sigma_{\theta} \bar{\wedge} \sigma_{\varphi}=\left(-\sin ^{2} \varphi \cos \theta,-\sin ^{2} \varphi \sin \theta,-\sin \varphi \cos \varphi\right)
$$

for those points of the supporting oriented Riemann contact sphere $\mathbb{S}_{2}$ which are encoded by the rectangular coordinates $(\theta, \varphi) \in] 0,2 \pi[\times] 0, \pi[$. In particular, the de Coriolis alternating trilinear form implies that with respect to the outward-pointing orientation of the two-dimensional compact spin manifold $\mathbb{S}_{2}$, the geodesic curvature form $k_{\gamma}$ of the temporally parametrized $\varphi$-latitudinal smooth loop

$$
\gamma:] 0,2 \pi \sin \varphi\left[\ni t \rightsquigarrow \sigma\left(\frac{1}{\sin \varphi} t, \varphi\right)\right.
$$

is constant at

$$
k_{\gamma}=\cot \varphi
$$


where $\varphi \in] 0, \pi$ [ denotes the latitudinal direction angle of the swivel. The real-valued piecewise smooth angular gauge function $t \rightsquigarrow \theta(t)$ of the smooth loop $\gamma$ satisfies the skew-differential equation of $\varphi$-latitudinal dynamics

$$
\theta^{\prime}=-k_{\gamma}
$$

with phase shift $\theta_{0}=0$, and $\varphi$-latitudinal phase angle displacement

$$
\Delta \theta=-\int_{0}^{2 \pi \sin \varphi} \theta^{\prime}(s) \mathrm{d} s
$$

beneath the swivel and above the terrestrial circular floor panel $\mathcal{O}_{\infty}$ at the $\varphi$-latitudinal direction angle $\varphi \in] 0, \pi$ [. The Laguerre contact geometry affords for the dynamics of the Foucault spherical pendulum:

Theorem 20 Let $\gamma$ denote the unit-velocity parametrization of the $\varphi$-latitudinal smooth loop enclosing the spherical contact cap surface $\mathcal{O}_{\varphi}$ which is contained in the two-dimensional compact spin manifold $\mathbb{S}_{2}$ of the complex projective manifold $\mathbb{P}_{\mathbb{C}}\left(\mathfrak{L} \mathfrak{i}(\mathcal{N})^{*}\right)$. The $\varphi$-latitudinal phase angle displacement $\Delta \theta$ of the spin echo-stabilized, symplectic swing-plane of Foucault's spherical pendulum holonomy experiment around the loop $\gamma$ is given in terms of the constant geodesic curvature form at $k_{\gamma}=\cot \varphi$ with respect to the outward-pointing orientation of the two-dimensional compact homogeneous spin manifold $\mathbb{S}_{2} \cong \mathrm{SO}(3, \mathbb{R}) / \mathrm{SO}(2, \mathbb{R})$ by the coboundary relation of the double diurnal rotation tracking of the projectively stellarized spinning earth

$$
\Delta \theta=\int_{0}^{2 \pi \sin \varphi} k_{\gamma}(s) \mathrm{d} s=2 \pi-\operatorname{area}\left(\mathcal{O}_{\varphi}\right)=2 \pi \cos \varphi
$$

where $\varphi \in] 0, \pi[$ denotes the $\varphi$-latitudinal direction angle of the swivel at the projective choice of spinor gauge of relativistic gravitation in the circular contact cone calibration of navigation.

As a conclusion the following statements pop up::

Corollary 1 The contact holonomy around the unit-velocity $\varphi$-latitudinal smooth loop $\gamma$ and marked by the diameter of the spin structurized conformal hyperbolic open disc model, is equal to a clockwise rotation of the tangent plane $\mathrm{T}_{\gamma(\bullet)} \mathbb{S}_{2}$ supporting the twodimensional compact spin manifold $\mathbb{S}_{2} \hookrightarrow \mathbb{P}_{\mathbb{C}}\left(\mathfrak{L} \mathfrak{i}(\mathcal{N})^{*}\right)$ by the $\varphi$-latitudinal phase angle displacement $\Delta \theta$ of the parallel transport dynamics of spin echo-stabilized, symplectic swing-planes.

The pair of circular contact cones of nutation in the complex projective manifold $\mathbb{P}_{\mathbb{C}}\left(\mathfrak{L} \mathfrak{i} \mathfrak{e}(\mathcal{N})^{*}\right)$ defines the paradigma of contact calibration of parallel transport or parallel translation of the initial, symplectic swing-plane around a $\varphi$-inclined Villarceau circular fiber $\gamma$ on the phase framing gyroscopic contact torus of revolution $\mathbb{S}_{1} \times \mathbb{S}_{1}$ of genus one with central circle of nutation inside the solid torus. It gives rise to a contact 
holonomy theorem of projective Ambrose-Singer type in the complex projective manifold $\mathbb{P}_{\mathbb{C}}\left(\mathfrak{L i} \mathfrak{i}(\mathcal{N})^{*}\right)$ in terms of the real-valued angular gauge function $t \rightsquigarrow \theta(t)$ and the geodesic curvature form $k_{\gamma}$ at latitude direction angle $\left.\varphi \in\right] 0, \pi$ [ of the principal circle bundle $\mathrm{SO}(3, \mathbb{R}) \longrightarrow \mathrm{SO}(3, \mathbb{R}) / \mathrm{SO}(2, \mathbb{R})$ over the two-dimensional compact spin manifold $\mathbb{S}_{2} \hookrightarrow \mathbb{P}_{\mathbb{C}}\left(\mathfrak{L i e}(\mathcal{N})^{*}\right)$, whereas the metrical version of the classical holonomy theorem of Ambrose and Singer describes the relation between holonomy and curvature of Riemannian manifolds.

Corollary 2 In accordance with the Witt index 2, parallel velocity vector fields around the equatorial great circle contained in the two-sided homogeneous plane at infinity $\mathcal{O}_{\infty} \hookrightarrow \mathbb{P}_{\mathbb{C}}\left(\mathfrak{L i e}(\mathcal{N})^{*}\right)$ at latitude drection angale $\varphi=\frac{1}{2} \pi$ imply the vanishing $\varphi$-latitudinal phase angle displacement $\Delta \theta=0$ of the spin echo-stabilized, symplectic swing-plane of the supplementary open-book foliation of the Foucault spherical pendulum holonomy experiment.

The calculation of the base version of the covariant derivative $-k_{\gamma} \gamma^{\prime}$ of the Hopf principal circle bundle $\mathbb{S}_{1} \hookrightarrow \mathbb{S}_{3} \stackrel{\eta_{2}}{\longrightarrow} \mathbb{S}_{2}$ around $\gamma$ in the supporting oriented Riemann contact sphere $\mathbb{S}_{2} \hookrightarrow \mathbb{P}_{\mathbb{C}}\left(\mathfrak{L} \mathfrak{i} \mathfrak{e}(\mathcal{N})^{*}\right)$ uses the fact that the real-valued angular gauge function $t \rightsquigarrow \theta(t)$ satisfies the skew-differential equation $-k_{\gamma}=\theta^{\prime}$ which describes $\gamma^{\prime}$ in terms of a parallel vector field. It means that the geodesic curvature form $k_{\gamma}$ measures the rate of turning of its velocity vector, reckoned with respect to a parallel vector field, annihilated by the covariant derivative operator $\nabla$, along $\gamma$. Applied to the restriction

$$
\left.\sigma_{\varphi} \mid \gamma:\right] 0,2 \pi \sin \varphi\left[\ni t \rightsquigarrow \sigma_{\varphi}\left(\frac{1}{\sin \varphi} t, \varphi\right),\right.
$$

the covariant derivative $\nabla\left(\sigma_{\varphi} \mid \gamma\right)$ of the velocity yields the orthogonal vector

$$
-k_{\gamma} \gamma^{\prime} \perp \sigma_{\varphi} \mid \gamma
$$

of the supplementary open-book foliation. If the unit-velocity curve $\gamma$ is a geodesic trajectory in a two-dimensional oriented connected complete smooth manifold $\mathcal{M}$, then $k_{\gamma}=0$ so that the real-valued angular gauge function $t \rightsquigarrow \theta(t)$ is constant. Thus a unit-length vector field along $\gamma$ is parallel if and only if it maintains a constant angle with the tangent vector field $\gamma^{\prime}$ on the manifold $\mathcal{M}$. In particular, the vector field $\gamma^{\prime}$ is parallel on $\mathcal{M}$. In case of the supporting oriented Riemann contact sphere $\mathcal{M}=\mathbb{S}_{2} \hookrightarrow \mathbb{P}_{\mathbb{C}}\left(\mathfrak{L i e}(\mathcal{N})^{*}\right)$, the restriction $\sigma_{\varphi} \mid \gamma$ defines a parallel vector field of unit length if and only if the coordinate $\varphi=\frac{1}{2} \pi$ is the latitude direction angle of the equatorial great circle of the two-dimensional compact spin manifold $\mathbb{S}_{2} \cong \mathbb{P}_{1}(\mathbb{C})$.

Remark 8 The preceding theorem on contact cone calibration of the double diurnal rotation tracking of the projectively stellarized spinning earth is in analogy to the second Kepplerian spinor law of the contact geometry of planetary dynamics. The focal position of the immobile sun is defined by the orbital ellipse which is definitely not a circular orbit in the complex projective manifold $\mathbb{P}_{\mathbb{C}}\left(\mathfrak{L i \mathfrak { e }}(\mathcal{N})^{*}\right)$. 
As a consequence of the contact holonomy theorem of projective Ambrose-Singer type, the projective choice of spinor gauge of relativistic gravitation together with the real-valued angular gauge function $t \rightsquigarrow \theta(t)$ complete the intrinsic description of the diurnal rotation per siderial day of the spinning earth and its projective stellarization within the complex projective manifold $\mathbb{P}_{\mathbb{C}}\left(\mathfrak{L} \mathfrak{i} \mathfrak{e}(\mathcal{N})^{*}\right)$ of quantum field theory.

Corollary 3 Due to the Witt index 2 , the spin group $\operatorname{Spin}(2, \mathbb{R})$ forms the two-fold covering of the holonomy group $\mathrm{SO}(2, \mathbb{R})$ consisting of the $\varphi$-latitudinal parallel transport maps $\Delta \theta$ around the piecewise smooth loops $\gamma$ of the two-dimensional compact homogeneous spin manifold $\mathbb{S}_{2} \cong \mathrm{SO}(3, \mathbb{R}) / \mathrm{SO}(2, \mathbb{R})$.

The contact holonomy of the tangent bundle of the standard Riemannian metric on the two-dimensional compact spin manifold $\mathbb{S}_{2}$ is the group $\operatorname{SO}(2, \mathbb{R})$. It follows that signature isomorphism WQ $(\mathbb{R}) \longrightarrow \mathbb{Z}$ of the wideband Witt group over the field of central projective collineations $\operatorname{PSO}(1,1, \mathbb{R}) \cong \mathbb{R}$ is the natural spectral companion of the embedding spin group $\operatorname{Spin}(3, \mathbb{R}) \cong \mathbb{S}_{3}$ which reveals the procedure of contact cone calibration to be equivalent to the Plancherel version of the Poisson summation formula of Abelian Fourier analysis.

The two-fold fibration of the phase framing gyroscopic contact torus of revolution $\mathbb{S}_{1} \times \mathbb{S}_{1}$ by pairs of coherent Villarceau phase circles $\widehat{\mathrm{WQ}}(\mathbb{R})$ under opposite orientation proves by Sagnac interference:

Theorem 21 The monodromy group of the principal circle bundle $\mathrm{SO}(3, \mathbb{R}) \longrightarrow$ $\mathrm{SO}(3, \mathbb{R}) / \mathrm{SO}(2, \mathbb{R})$ over the simply connected compact spin manifold $\mathbb{S}_{2} \hookrightarrow$ $\mathbb{P}_{\mathbb{C}}\left(\mathfrak{L i} \mathfrak{i}(\mathcal{N})^{*}\right)$ is trivial.

One of the founders of contact topology has been the cartographer Johann Heinrich Lambert (1728-1777) who succeeded in the mathematical construction of an equiareal mapping from the round sphere to its contact cylinder [61]. Specifically, the Lambert projector of the earth is obtained by projecting the terrestrial globe onto the cylinder via equiareally shadowing, and then unwrapping the cylinder into a flat rectangular lattice designed by the horizontal action of the free Abelian Witt group $\mathrm{WQ}(\mathbb{R}) \cong \mathbb{Z}$ over the field of central projective collineations $\operatorname{PSO}(1,1, \mathbb{R}) \cong \mathbb{R}$. Keppler orbital ellipses centered at the grid points help to visualize the separate vertical and horizontal expansion-contraction ratios of the equiareal cartographic projection. The orbital ellipses are images of equalized circles under the derivative of the projection from globe to map at a lattice of equally spaced points along the mapping.

In addition to his cartographic work, the circling of Villarceau circles within circles, the perfect functioning of a clocklike flat torus device in the very round sphere model $\mathbb{S}_{3} \hookrightarrow \mathbb{R}^{4}$, was first described "in quarto dimensions" by Lambert within the "Cosmological Letter" sent to Kant in 1765. Lambert's projective approach to the ordo orbium proposed to Kant "the very same theory of the systematic constitution of the worldedifice as a whole". Due to the projective functor $\mathbb{P}(\bullet)$, space had gained depth but had lost structure. Lambert's treatise Perspectiva problemata inversa of 1756 actually can be viewd as a reconstruction guide for the underlying Witt group WQ(R) and its stellarization ladder which is zooming the spinor gauge by the signature isomorphism $\mathrm{WQ}(\mathbb{R}) \longrightarrow \mathbb{Z}$. His repeated reflection that "perhaps we are not sufficiently Copernican" sums up man's "removal from the center of the universe" and the endeavour 
to projectively subsume spinning super solar systems like galaxies into more comprehensive relativistic systems that are less constrained by man's initial point of view and extended by the projective geometry of invariance group $\operatorname{PSO}(1,3, \mathbb{R})$.

\section{By way of conclusion}

Despite significant recent progress, the preparation of quantum superposition states remains outstanding due to weak coupling and thermal decoherence phenomena. When applied to a low temperature state, the affine tomographic platform of the post-Kepplerian theorem of Alexandrov-Ovchinnikova highlights the generation of quantum compass states, which are superpositions of four coherent states at cardinal positions in phase space. These highly non-classical quantum states have very sharp phase-space features and are of considerable interest for quantum information and sensing applications to the outcome of quantum channels [37].

Due to the equivalence principle of gravitational and inertial mass, the rotationsensing technologies of ring laser gyroscopes and miniaturized fiber optic gyroscopes support inertial navigation strategies. Taking advantage of the development of optical fiber communication technologies, the fiber optic gyroscope started to be investigated in the mid-1970s, opening the way for a fully solid-state rotation sensor. The fiber optic gyroscope was firstly seen as dedicated to medium-grade applications of communication channels, but today, it reaches strategic-grade performance and surpasses its well-established competitor, the ring laser gyroscope, in terms of bias noise and longterm spin echo-stability. Further progress remains possible, the challenge being the ultimate inertial navigation performance of one nautical mile per month corresponding to a long-term spin echo-stability.

Acknowledgements Open Access funding provided by Projekt DEAL. The author is grateful to the ARC Centre of Excellence for Engineered Quantum Systems of the University of Queensland, Australia, for providing Figure 4 supra.

Open Access This article is licensed under a Creative Commons Attribution 4.0 International License, which permits use, sharing, adaptation, distribution and reproduction in any medium or format, as long as you give appropriate credit to the original author(s) and the source, provide a link to the Creative Commons licence, and indicate if changes were made. The images or other third party material in this article are included in the article's Creative Commons licence, unless indicated otherwise in a credit line to the material. If material is not included in the article's Creative Commons licence and your intended use is not permitted by statutory regulation or exceeds the permitted use, you will need to obtain permission directly from the copyright holder. To view a copy of this licence, visit http://creativecommons.org/licenses/by/4.0/.

\section{References}

1. Alexandrov, A.D., Ovchinnikova, V.V.: Notes on the foundations of relativity theory. Vestnik Leningrad. Univ. 111, 95-110 (1953)

2. Alexandrov, A.D.: Mappings of spaces with families of cones and space-time transformations. Annali di Matematica Pura ed Applicata 193, 229-257 (1975)

3. Amaral, D.G., Witter, M.P.: The three-dimsensional organization of the hippocampal formation: a review of anatomical data. Neuroscience 31, 571-591 (1989) 
4. Andersen, P., Morris, R., Amaral, D., Bliss, T., O’Keefe, J. (eds.): The Hippocampus Book. Oxford University Press, Oxford, New York (2007)

5. Artin, E.: Geometric Algebra. Interscience Publishers, New York (1957)

6. Baez, J.C.: The octonions. Bull. (New Ser.) Am. Math. Soc. 39, 145-205 (2001)

7. Barton, S., Kean, M., Harvey, S., Yang, J.: Advanced neuroimaging and pediatric epilepsy surgery. MAGNETOM Flash 73(2), 49-57 (2019)

8. Basser, P.J., Pajevic, S., Pierpaoli, C., Duda, J., Aldroubi, A.: In vivo fiber tractography using DT-MRI data. Magn. Reson. Med. 44(4), 625-632 (2000)

9. Bernstein, M.A., King, K.F., Zhou, X.J.: Handbook of MRI Pulse Sequences. Elsevier Academic Press, Amsterdam (2004)

10. Chevalley, C.: The Algebraic Theory of Spinors and Clifford Algebras. Collected Works, vol. 2. Springer, Berlin (1997)

11. Dietrich, O.: Diffusion-weighted imaging and diffusion tensor imaging. In: Reiser, M.F., Semmler, W., Hricak, H. (eds.) Magnetic Resonance Tomography, pp. 130-152. Springer, Berlin (2008)

12. Duvernoy, H.M., Cattin, F., Risold, P.-Y.: The Human Hippocampus: Functional Anatomy, Vascularization and Serial Sections with MRI, 4th edn. Springer, Berlin (2013)

13. Eichenbaum, H., Wiener, S.I., Shapiro, M.L., Cohen, N.J.: The organization of spatial coding in the hippocampus: a study of neural ensemble activity. J. Neurosci. 9, 2764-2775 (1989)

14. Fiete, I.R., Burak, Y., Brookings, T.: What grid cells convey about RAT location. J. Neurosci. 28, 6858-6871 (2008)

15. Fyhn, M., Molden, S., Witter, M.P., Moser, E.I., Moser, M.-B.: Spatial representation in the entorhinal cortex. Science 305, 1258-1264 (2004)

16. Fyhn, M., Hafting, T., Treves, A., Moser, M.-B., Moser, E.I.: Hippocampal remapping and grid realignment in entorhinal cortex. Nature 446, 190-194 (2007)

17. Gras, G.: Class Field Theory: From Theory to Practice. Springer, Berlin (2005)

18. Griffiths, P.: Variations on theorem of Abel. Invent. Math. 35, 321-390 (1976)

19. Hafting, T., Fyhn, M., Molden, S., Moser, M.-B., Moser, E.I.: Microstructure of a spatial map in the entorhinal cortex. Nature 436, 801-806 (2005)

20. Higgins, J.R.: Five short stories about the cardinal series. Bull. Am. Math. Soc. (New Ser.) 12, 45-89 (1985)

21. Johansen-Berg, H., Behrens, T.E.J. (eds.): Diffusion MRI: From Quantitative Measurement to In vivo Neuroanatomy. Elsevier, Academic Press, Amsterdam, Boston, Heidelberg (2009)

22. Jones, D.K. (ed.): Diffusion MRI: Theory, Methods, and Applications. Oxford University Press, Oxford (2010)

23. Khan, U.A., Liu, L., Provenzano, F.A., Berman, D.E., Profaci, C.P., Sloan, R., Mayeux, R., Duff, K.E., Small, S.A.: Molecular drivers and cortical spread of lateral entorhinal cortex dysfunction in preclinical Alzheimer's disease. Nat. Neurosci. 17, 304-311 (2014)

24. Le Bihan, D. (ed.): Diffusion and Perfusion Magnetic Resonance Imaging: Applications to Functional MRI. Raven Press, New York (1995)

25. Lion, G., Vergne, M.: The Weil Representation. Maslov Index and Theta Series. Birkhäuser Verlag, Boston (1980)

26. Lorimer, D.R., Kramer, M.: Handbook of Pulsar Astromomy. Cambridge University Press, Cambridge (2005)

27. Lyne, A.G., Graham-Smith, F.: Pulsar Astronomy, 3rd edn. Cambridge University Press, Cambridge (2005)

28. Maier-Hein, K.H., Neher, P.F., Houde, J.C., Côté, M.A., Garyfallidis, E., Zhong, J., et al.: The challenge of mapping the human connectome based on diffusion tractography. Nat. Commun. 8(1), 1349 (2017)

29. Mori, S.: Introduction to Diffusion Tensor Imaging. Elsevier, Amsterdam (2007)

30. Moritani, T., Ekholm, S., Westesson, P.-L.A.: Diffusion-Weighted MR Imaging of the Brain, 2nd edn. Springer, Berlin (2009)

31. Müller, C.A.: Diffusive spin transport. In: Buchleitner, A., Viviescas, C., Tiersch, M. (eds.) Entanglement and Decoherence: Foundations and Modern Trends. Lecture Notes in Physics, vol. 768, pp. 277-314. Springer, Berlin (2010)

32. Mueller, L., Tax, C.M.W., Jones, D.K.: Unprecedented echo times for diffusion MRI using connectom gradients, spiral readouts and field monitoring. MAGNETOM Flash 74(3), 21-26 (2019) 
33. Naidich, T.P., Daniels, D.L., Haughton, V.M., Williams, A., Pojunas, K., Palacios, E.: Hippocampal formation and related structures of the limbic lobe: anatomic-MR correlation I. Surface features and coronal sections. Radiology 162, 747-754 (1987)

34. Ockeloen, C.F., Schmied, R., Riedel, M.F., Treutlein, P.: Quantum metrology with a scanning probe atom interferometer. Phys. Rev. Lett. 111, 143991 (2013)

35. O'Keefe, J., Nadel, L.: The Hippocampus as a Cognitive Map. Oxford University Press, Oxford (1978)

36. O'Keefe, J., Burgess, N.: Dual phase and rate coding of hippocampal place cells: theoretical significance and relationship in entorhinal grid cells. Hippocampus 15, 853-866 (2005)

37. Ozorio de Almeida, A.M.: Entanglement in phase space. In: Buchleitner, A., Viviescas, C., Tiersch, M. (eds.) Entanglement and Decoherence: Foundations and Modern Trends. Lecture Notes in Physics, vol. 768, pp. 157-219. Springer, Berlin (2010)

38. Pezzè, L., Smerzi, A.: Ultrasensitive two-mode interferometry with single-mode number squeezing. Phys. Rev. Lett. 110, 16304 (2013)

39. Raza, H., Hayat, S., Pan, X.-F.: On the fault-tolerant metric dimension of certain interconnection networks. J. Appl. Math. Comput. 60, 517-535 (2019)

40. Ringbauer, M., Weinhold, T.J., Howard, L.A., White, A.G., Vanner, M.R.: Generation of mechanical interference fringes by multi-photon counting. New J. Phys. 20, 053042 (2018)

41. Saito, M.: Représentations unitaires des groupes symplectiques. J. Math. Soc. Jpn. 24, 232-251 (1972)

42. Salzmann, H., Betten, D., Grundhöfer, T., Hähl, H., Löwen, R., Stroppel, M.: Compact Projective Planes. With an Introduction to Octonion Geometry. Walter de Gruyter, Berlin (1995)

43. Scharlau, W.: Quadratic and Hermitian Forms. Springer, Berlin (1985)

44. Schempp, W.J.: Magnetic Resonance Imaging: Mathematical Foundations and Applications. Wiley, New York (1998)

45. Schempp, W.J.: Dynamic metaplectic spinor quantization: the projective correspondence for spectral dual pairs. J. Appl. Math. Comput. 59, 545-584 (2019)

46. Schempp, W.J.: Galois cohomology and the metaplectic Schaar-Landsberg construction. Constr. Math. Anal. (to appear)

47. Schmitt, F., Stehling, M.K., Turner, R.: Echo-Planar Imaging: Theory. Technique and Application. Springer, Berlin (1998)

48. Schneider, J.F., Il'yasov, K.A., Henning, J., Martin, E.: Fast quantitative diffusion-tensor imaging of cerebral white matter from the neonatal period to adolescence. Neuroradiology 46, 258-266 (2004)

49. Schreiber, S., Erchova, I., Heinemann, U., Herz, A.V.M.: Subthreshold resonance explains the frequency-dependent integration of periodic as well as random stimuli in the entorhinal cortex. J. Neurophys. 92, 408-415 (2004)

50. Schwartz, R.E.: The Poncelet grid. Adv. Geom. 7, 157-175 (2007)

51. Serre, J.-P.: Galois Cohomology. Springer, Berlin (2002)

52. Solodkin, A., Van Hoesen, G.W.: Entorhinal cortex modules of the human brain. J. Comparat. Neurol. 365, 610-627 (1996)

53. Steffenach, H.-A., Witter, M.P., Moser, M.-B., Moser, E.I.: Spatial memory in the rat requires the dorsolateral band of the entorhinal cortex. Neuron 45, 301-313 (2005)

54. Stejskal, E.O.: Use of spin echoes in a pulsed magnetic-field gradient to study non-isotropic, restricted diffusion and flow. J. Chem. Phys. 43, 3597-3603 (1965)

55. Stejskal, E.O., Tanner, J.E.: Spin diffusion measurements: Spin echoes in the presence of a timedependent field gradient. J. Chem. Phys. 42, 288-292 (1965)

56. Stephenson, B.: Kepler's Physical Astronomy. Princeton University Press, Princeton (1994)

57. Stieltjes, B., Brunner, R.M., Fritzsche, K.H., Laun, F.B.: Diffusion Tensor Imaging: Introduction and Atlas. Springer, Berlin (2013)

58. Suzuki, W.A.: Perception and the medial temporal lobe: evaluating the current evidence. Neuron 61, 657-666 (2009)

59. Suzuki, W.A., Amaral, D.G.: Functional neuroanatomy of the medial temporal lobe memory system. Cortex 40, 220-222 (2004)

60. Tanner, J.E., Stejskal, E.O.: Restricted self-diffusion of protons in colloidal systems by the pulsedgradient, spin echo method. J. Chem. Phys. 49, 768-777 (1968)

61. Tapp, K.: Differential Geometry of Curves and Surfaces. Springer, Berlin (2016)

62. Tournier, J.D., Calamante, F., Gadian, D.G., Connelly, A.: Direct estimation of the fiber orientation density function from diffusion-weighted MRI data using spherical deconvolution. Neuroimage 23, 1176-1186 (2004) 
63. Tournier, J.D., Yeh, C.H., Calamante, F., Cho, K.H., Connelly, A., Lin, C.P.: Resolving crossing fibers using constrained spherical deconvolution: validation using diffusion-weighted imaging phantom data. Neuroimage 42, 617-625 (2008)

64. Van Hecke, W., Emsell, L., Sunaert, S. (eds.): Diffusion Tensor Imaging: A Practical Handbook. Springer, New York (2016)

65. Van Hoesen, G.W., Solodkin, A.: Some modular features of temporal cortex in humans as revealed by pathological changes in Alzheimer's disease. Cerebral Cortex 3, 465-475 (1993)

66. Villeneuve, D.M., Hockett, P., Vrakking, M.J.J., Nikura, H.: Coherent imaging of an attosecond electron wave packet. Science 356, 1150-1153 (2017)

67. Warach, S., Gaa, J., Siewert, B., Wielopolski, P.A., Edelman, R.R.: Acute human stroke studied by whole brain echo planar diffusion-weighted magnetic resonance imaging. Ann. Neurol. 37, 231-241 (1995)

68. Weil, A.: Basic Number Theory, 3rd edn. Springer, Berlin (1974)

69. Weil, A.: Sur certains groupes d'opérateurs unitaires. Acta Math. 111, 143-211 (1964), Collected Papers, Vol. III, pp. 1-69, Springer-Verlag, New York, Heidelberg, Berlin 1979

70. Weil, A.: Elliptic functions according to Eisenstein and Kronecker. Springer, Berlin (1999)

71. Weisberg, J.M., Nice, D.J., Taylor, J.H.: Timing measurements of the relativistic binary pulsar PSR B1913 + 16. Astrophys. J. 722, 1030-1034 (2010)

72. Weisberg, J.M., Taylor, J.H.: General relativistic geodetic spin precession in binary pulsar B1913 + 16 mapping the emission beam in two dimensions. Astrophys. J. 576, 942-949 (2002)

73. Weisberg, J.M., Huang, Y.: Relativistic measurements from timing the binary pulsar B1913 + 16. Astrophys. J. 829, 55 (2016)

74. Wiggins, C.J.: Why UHF MRI? A personal journey from artifact to anatomical history. MAGNETOM 71(2), 83-86 (2018)

75. Witter, M.P., Moser, E.I.: Spatial representation and the architecture of the entorhinal cortex. Trends Neurosci. 29, 671-678 (2006)

76. Wolf, F., Shi, C., Heip, J.C., Gessner, M., Pezzè, L., Smerzi, A., Schulte, M., Hammerer, K., Schmidt, P.O.: Motional Fock states for quantum-enhanced amplitude and phase measurements with trapped ions. Nat. Commun. 10, 1-8 (2019)

Publisher's Note Springer Nature remains neutral with regard to jurisdictional claims in published maps and institutional affiliations. 